\title{
3D printing of Continuous Fiber Reinforced Composites: A Review of the Processing, Pre- and Post-Processing Effects on Mechanical Properties
}

\author{
Faraz Safari, Abdolvahed Kami*, Vahid Abedini \\ Faculty of Mechanical Engineering, Semnan University, Semnan, Iran \\ *Corresponding Author's Email: akami@semnan.ac.ir
}

\begin{abstract}
The main objective of this study is to review existing research on the application of fused deposition modeling (FDM) for 3D printing of continuous fiber reinforced composites (CFRCs). An overview of additive manufacturing (AM) technology production techniques are provided first, followed by a look into FDM technology. The articles on CFRC printing were then summarized. The type of reinforcing material and matrix utilized, the research subject, the mechanical properties investigated, and the sample dimensions are all listed. Various pre-processing, processing, and post-processing conditions, as well as their impact on CFRC mechanical properties, were also discussed.
\end{abstract}

\section{Keywords:}

Additive manufacturing, 3D printing, Continuous fiber reinforced composites, Carbon fiber, Fused filament fabrication, Fused deposition modeling 


\section{Abbreviations}

Table 1. List of abbreviations

\begin{tabular}{|c|c|}
\hline AM & Additive Manufacturing \\
\hline 3DP & Three-Dimensional Printing \\
\hline ABS & Acrylonitrile Butadiene Styrene \\
\hline BIS & Beam Interference Solidification \\
\hline BPM & Ballistic Particle Manufacturing \\
\hline $\mathrm{CF}$ & Carbon Fiber \\
\hline CFRC & Continuous Fiber Reinforced Composites \\
\hline DMD & Direct Metal Deposition \\
\hline DMLS & Direct Metal Laser Sintering \\
\hline EBM & Electron Beam Melting \\
\hline FDM & Fused Deposition Modeling \\
\hline FFF & Fused Filament Fabrication \\
\hline FVF & Fiber Volume Fraction \\
\hline GF & Glass Fiber \\
\hline HIS & Holographic Interference Solidification \\
\hline IJP & Inkjet Printing \\
\hline KF & Kevlar Fiber \\
\hline LENS & Laser Engineered Net Shaping \\
\hline LOM & Laminated Object Manufacturing \\
\hline LPD & Laser Powder Deposition \\
\hline LTP & Liquid Thermal Polymerization \\
\hline
\end{tabular}




\begin{tabular}{|c|c|}
\hline MJM & Multijet Modeling \\
\hline PLA & Polylactic Acid \\
\hline POM & Polyoxymethylene \\
\hline SFP & Solid Foil Polymerization \\
\hline SGC & Solid Ground Curing \\
\hline SLA & Stereo Lithography \\
\hline SLC & Selective Laser Cladding \\
\hline SLM & Selective Laser Melting \\
\hline SLS & Selective Laser Sintering \\
\hline TPU & Thermoplastic Polyurethane \\
\hline
\end{tabular}

\section{Introduction}

As depicted in Fig. 1, formative manufacturing (injection molding, casting, stamping, and forging), subtractive manufacturing (milling, drilling, and turning), and additive manufacturing are the three types of manufacturing processes. The first two are traditional manufacturing technologies that have offered fewer attractions in recent decades when compared to additive manufacturing (AM) technology [1]. 


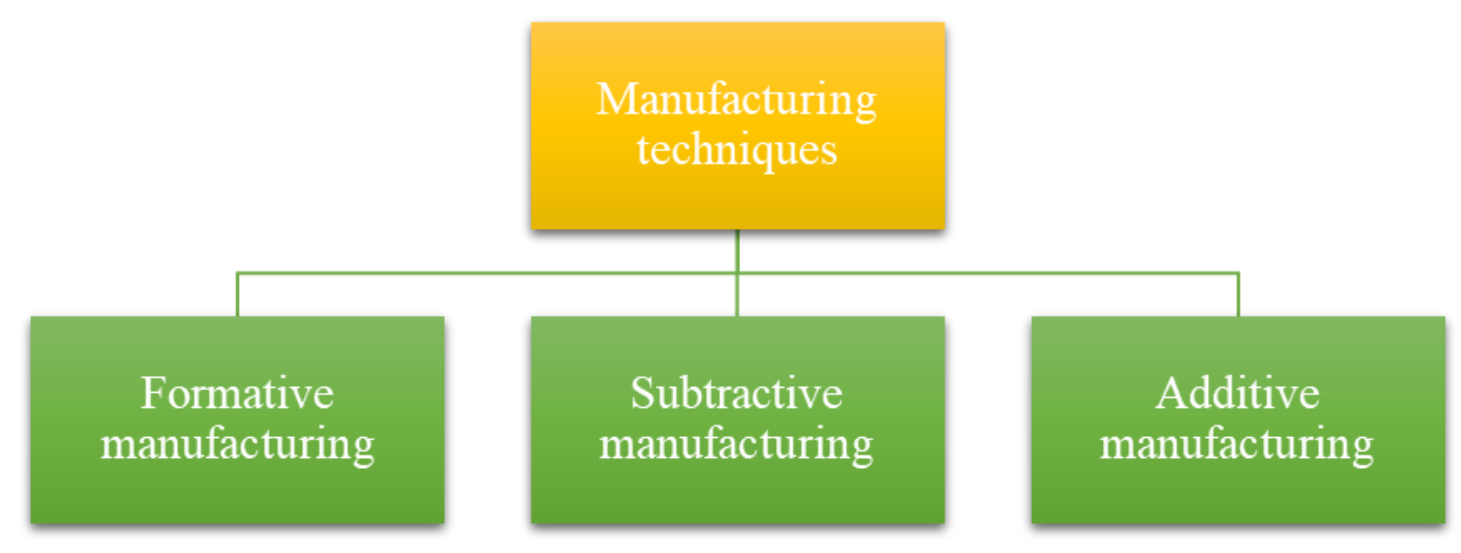

Fig. 1 Classification of manufacturing techniques

The layer-by-layer creation of an item from 3D model data utilizing multiple raw materials is referred to as AM technology, often known as 3D printing. In recent years, it has made significant development and is now regarded as a creative solution to many of the difficulties that traditional manufacturing methods confront. Its use is continuously developing, and the technology is improving regularly. AM decreases build time and cost, enhances operational flexibility, allows for quick prototyping, minimizes supply chain load, and, most significantly, can manufacture nearly anything that can be designed in CAD software. It's simple to predict that AM will be the obvious answer for designers and manufacturers all around the world in less than a decade. Aerospace [2, 3], automotive [3], manufacturing, treatment [4-6], research, architecture [7, 8], art [9], food [10-12], and apparel [13-15] are some of the applications of AM technology.

As illustrated in Fig. 2, AM processes are split into various categories. Powder, liquid, and solid (filament) are the basic materials utilized in 3D printers (as seen at the bottom of Fig. 2, with green for powder, blue for liquid, and red for solid). 


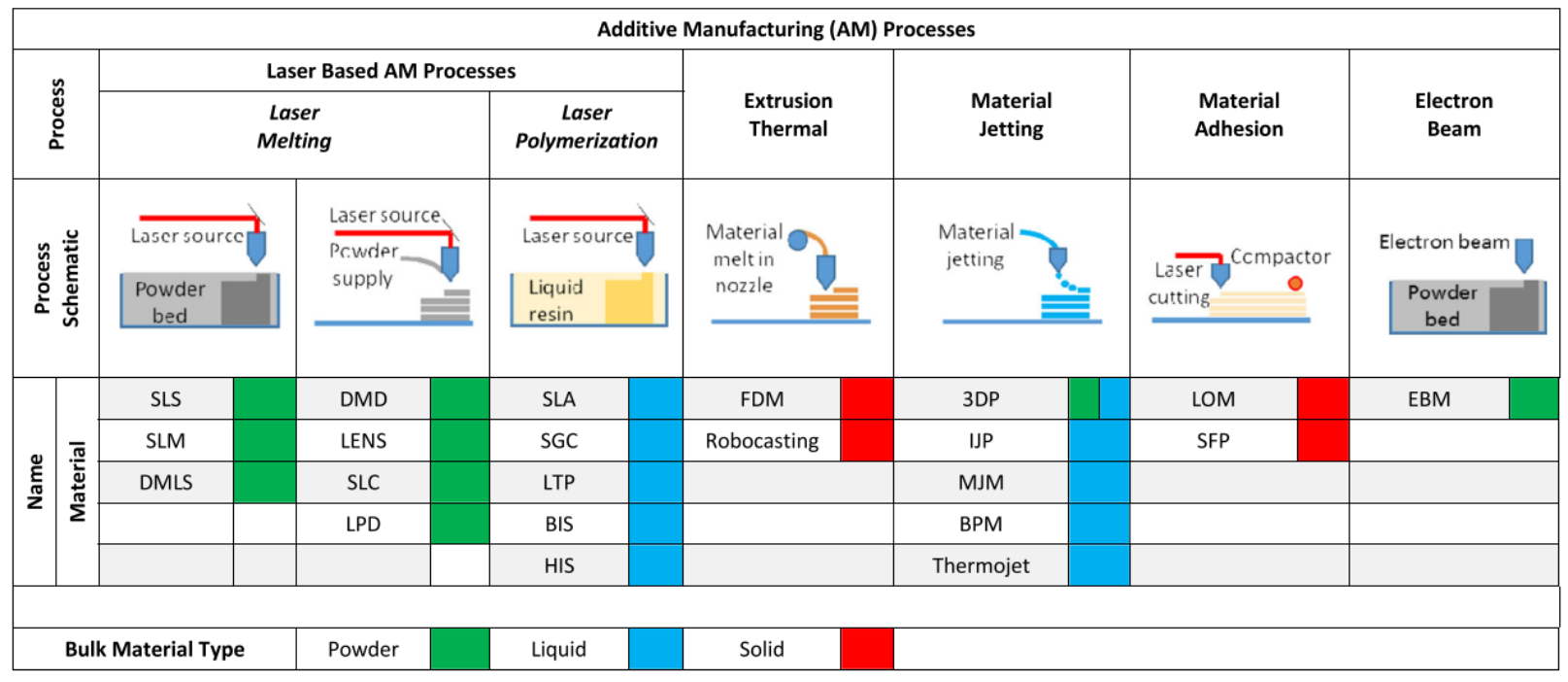

Fig. 2 Different categories of AM processes [16]

Laser melting methods include Selective Laser Sintering (SLS), Selective Laser Melting (SLM), Direct Metal Laser Sintering (DMLS), Electron Beam Melting (EBM), Laser Engineered Net Shaping (LENS), Direct Metal Deposition (DMD), Laser Powder Deposition (LPD), and Selective Laser Cladding (SLC). A laser source is used to selectively melt a material provided in the form of fine powder in laser melting AM techniques. After that, the material cools and solidifies to produce the final component. The laser beam is being guided in the $\mathrm{x}-\mathrm{y}$ plane by scanning optics, while a table travels in the z-direction. Laser polymerization, which includes prototyping technologies like Stereo Lithography (SLA), Solid Ground Curing (SGC), Liquid Thermal Polymerization (LTP), Beam Interference Solidification (BIS), and Holographic Interference Solidification (HIS), employs a liquid photosensitive resin that solidifies when illuminated by a (usually low-power) laser source. This disposable plastic material is used to create fine-cut pieces with a smooth surface for jewelry, dentistry, and medical applications. Extrusion processes include technologies such as Fused Deposition Modeling (FDM), Robocasting, and material extrusion 
techniques that employ a heated extrusion nozzle to soften or melt material, generally plastic, in the form of wire. After being melted, the material is extruded via an extrusion nozzle, which deposits the material before cooling to solidify and produce the final component shape. Material jetting technologies include Three-Dimensional Printing (3DP), Inkjet Printing (IJP), Multijet Modeling (MJM), Ballistic particle manufacturing (BPM), and Thermojet. Material jetting processes use multiple thin nozzles to "spray" either molten material or, more commonly, a binder (adhesive) to bind the powder in a solid object. The working concept of the process is similar to that of all laser-melting procedures, except that no phase transition occurs; instead, the binder binds the powder particles together. Adhesive-based processes include technologies such as Laminated Object Manufacturing (LOM), Solid Foil Polymerization (SFP), and others. However, adhesivebased processes are no longer widely used. The working concept comprises a cutter (often a laser) that cuts a thin coating of paper or plastic in the required outlines. The film is then pushed down over the preceding one by a heated compactor, activating a heat-curing adhesive located on the film's downward face, allowing it to be attached to the substrate. Electron beam procedures are similar to laser-melting processes, except that an electron beam is utilized as an energy source instead of a laser beam to melt or sinter the material.

A review of prior research on the manufacturing of continuous fiber reinforced composites (CFRCs) with FDM technology is provided in this publication. The influence of various preprocessing, processing (printing), and post-processing conditions on the mechanical properties of CFRCs has been investigated.

\section{Fused Deposition Modeling}

FDM technology, also known as FFF, is one of the most important AM methods that is based on solids and has become more desirable and attractive to industries and, most importantly, to the 
general public due to its simplicity, flexibility, fast prototyping, low cost, minimal waste, and ease of material change $[17,18]$. Because of their temperature characteristics, thermoplastic base filaments are the most commonly utilized in FDM. This technique may create components from thermoplastic filaments like PLA, ABS, Nylon, polypropylene (PP), polyether-ether-ketone (PEEK), and polyamides (PA) like PA6, PA12 [19-21]. This technique is a well-known technology that Crump [22] patented in 1989.

FDM 3D printing involves the deposit of a thermoplastic filament. A piece of filament from a reel is extruded in the XY plane, forming a layer of solid material on the build plate, after passing through a hot head at a temperature greater than the melting point of the filament. A model may be created by laying a layer shape and then filling the interior with plasticized material using a zigzag motion of the head. After printing one layer, the head travels along the Z-axis, causing the next layer to be built up. Using this approach, complicated forms with minimal preparation could be constructed [23]. The required item is developed in one CAD software, converted to an STL file, and then delivered to the machine for printing.

Using reinforcing fibers enhances strength and hardness, and these reinforcing fibers have excellent properties including high strength, lightweight, and anti-corrosion. As illustrated in Fig. 3, there are two forms of fiber-reinforced printing: short and continuous. The mechanical characteristics of CFRC (such as Young's modulus, tensile strength, and flexural strength) are significantly higher than those of short fiber reinforced or plain components (see Fig. 4). 


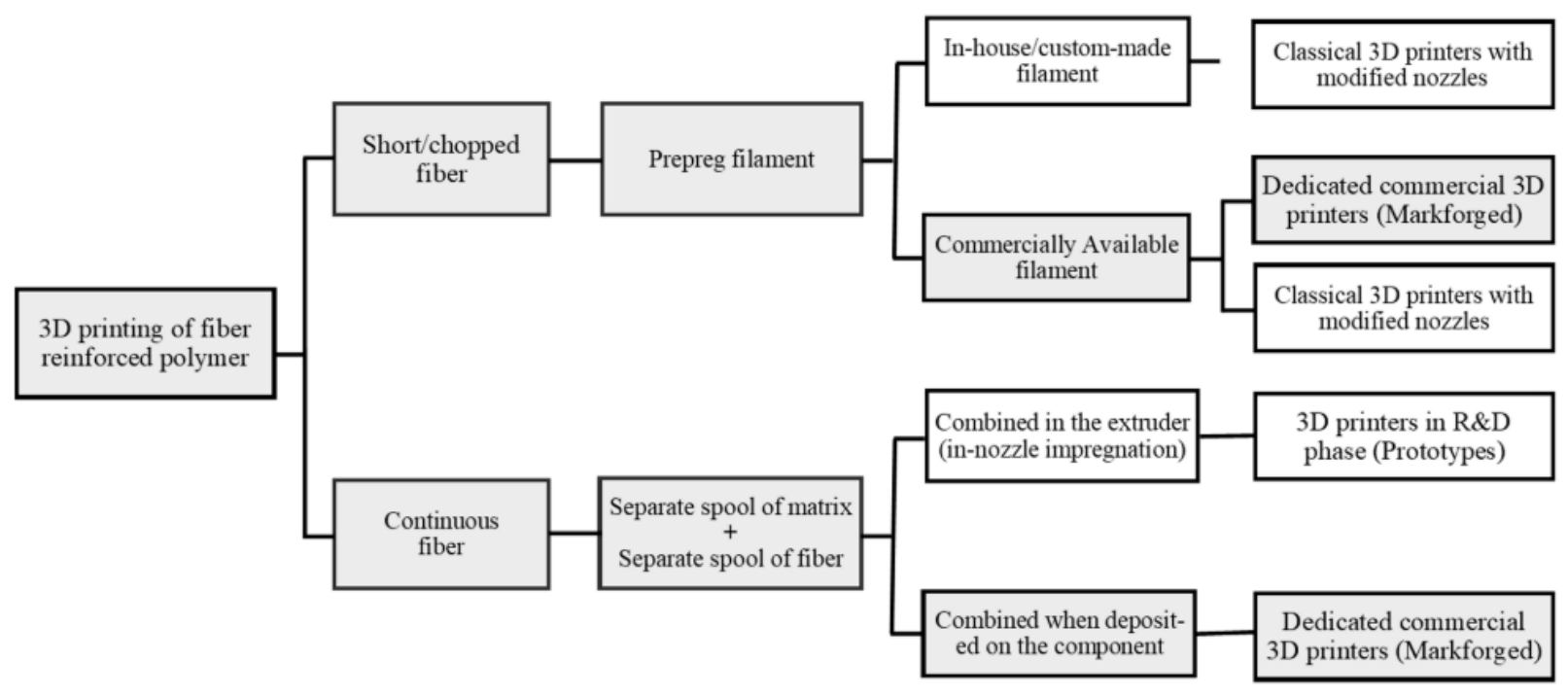

Fig. 3 3D-printed fiber-reinforced polymer manufacturing process classification [24] 

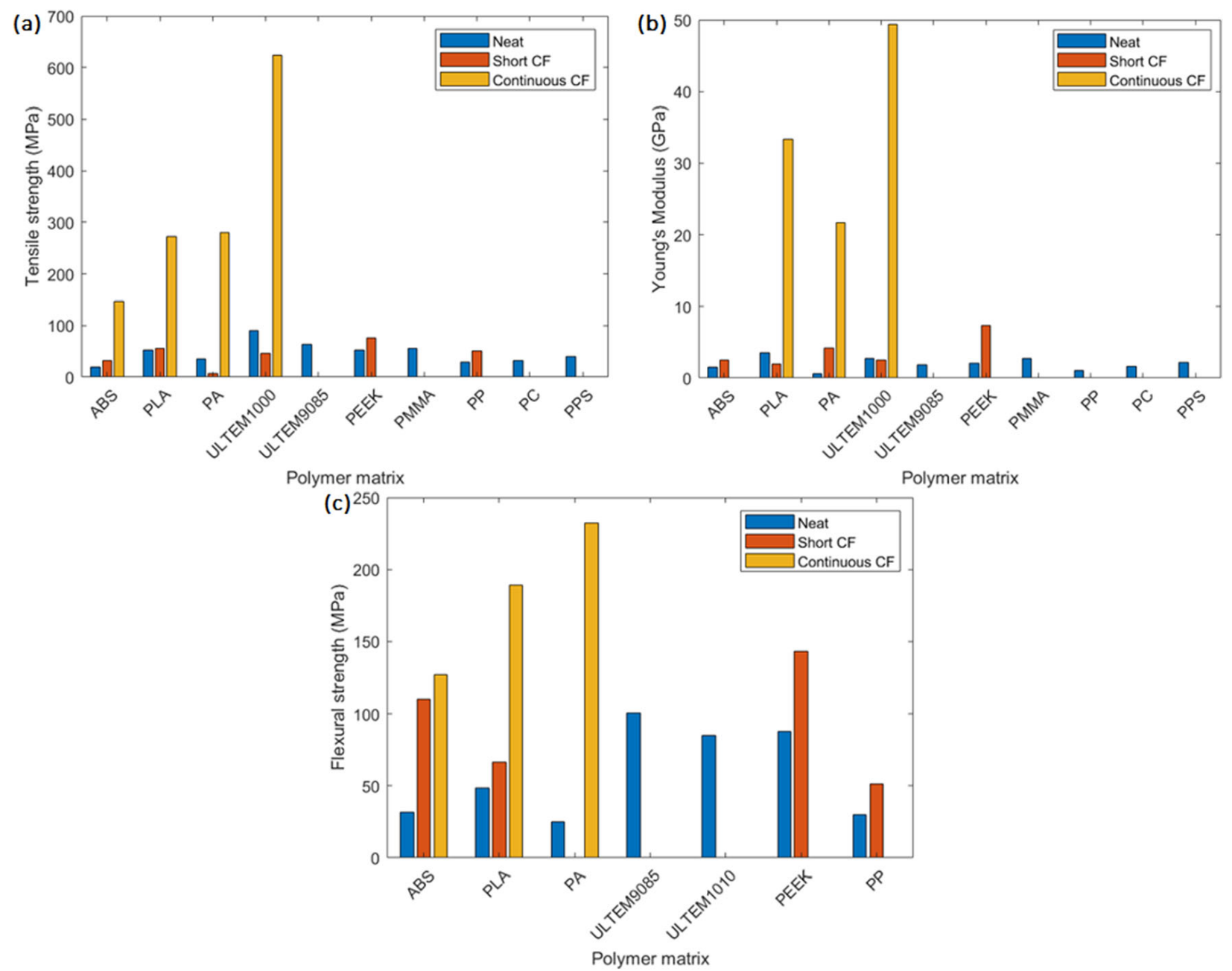

Fig. 4. Overall mechanical performance for FFF-produced specimens. Average (a) tensile strength, (b) Young's modulus, and (c) flexural strength values for neat, short CF reinforced, and continuous CF reinforced specimens [25]

\section{Mechanisms of Fiber-reinforced material's 3D printers}

Co-extrusion, dual extrusion, multi-degree of freedom, and compaction roller methods are four popular approaches for 3D printing CFRC [25-27]. Fig. 5 illustrates the schematics of these approaches. In the co-extrusion technique, the thermoplastic string and the reinforcing fiber are added separately to the head of the printing machine. In the dual extrusion method, the 
thermoplastic filament and the fiber-reinforced are extruded separately through two nozzles on the build platform [25]. The multi-degree of freedom method uses a robotic arm system to produce fiber-reinforced and thermoplastic [26]. In the compaction roller technique, a cartridge heater was secured to the nozzle body and used as a fixed shaft to support the compaction roller. The compaction roller had internal bearings to allow it to rotate freely around the cartridge heater.

The thermoplastic string and reinforcing fiber are introduced independently to the printing machine's head in the co-extrusion technique. The thermoplastic filament and the fiber-reinforced filament are extruded separately through two nozzles on the construction platform in the dual extrusion technique [25]. The multi-degree of freedom technique [26] makes fiber-reinforced and thermoplastic materials with a robotic arm system. In the compaction roller technique, a cartridge heater was attached to the nozzle body and utilized as a fixed shaft to support the compaction roller. Internal bearings allowed the compaction roller to freely spin around the cartridge heater. The compaction roller itself was heated by the cartridge heater [27]. 


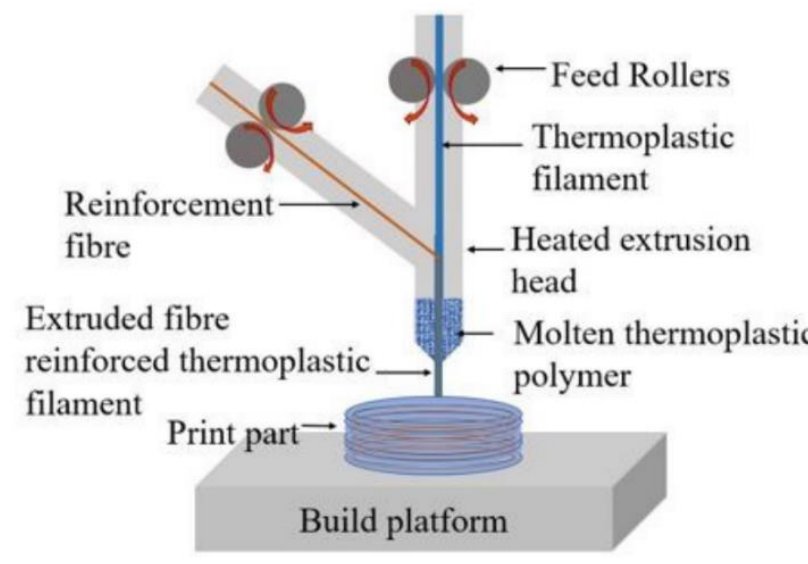

(a)

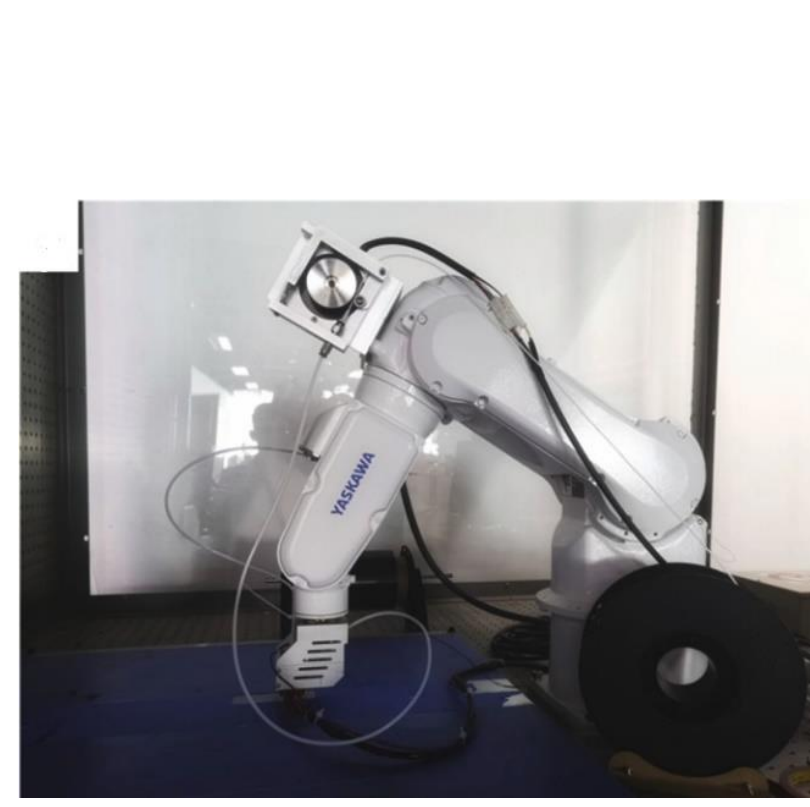

(c)

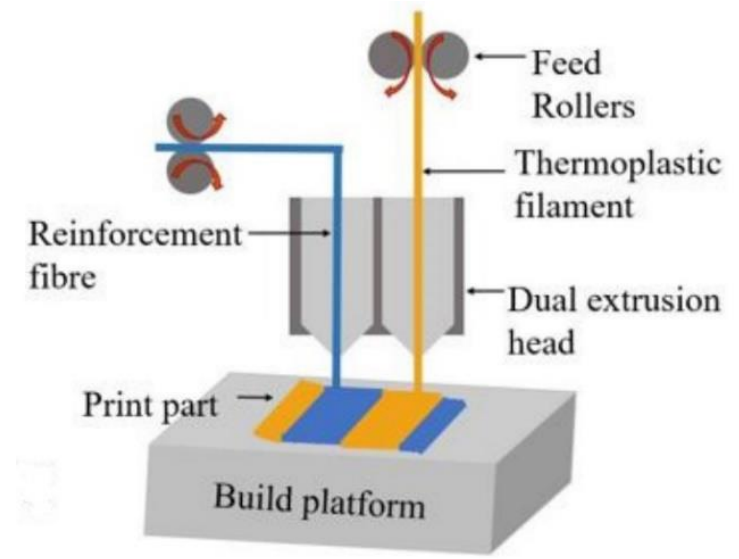

(b)

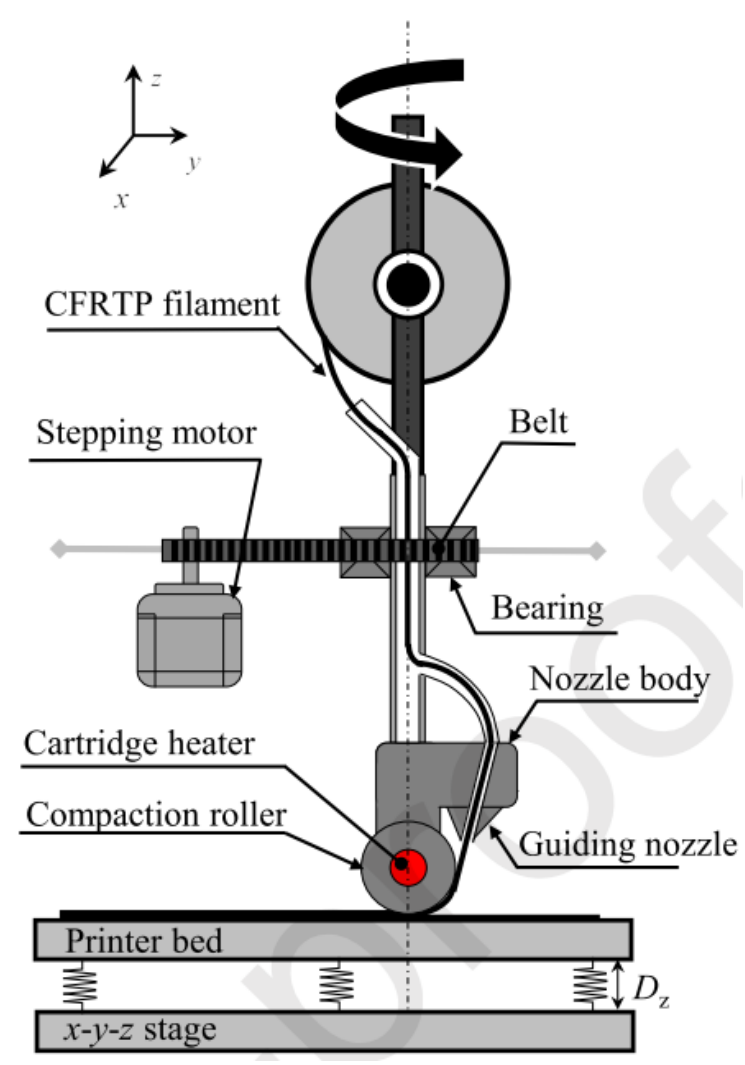

(d)

Fig. 5 Schematic illustration of the various mechanisms of 3D printers for printing fiberreinforced samples with (a) co-extrusion FDM printer [25], (b) dual-extrusion FDM printer [25], (c) multi-degree of freedom [26], and (d) compaction roller [27]. 


\section{An overview of CFRC 3D printing research}

In this article, the authors attempted to review all papers related to CFRC 3D printing. Table 2 summarizes these works and includes a list of the authors, publication year, study topic, matrix material, fiber-reinforced material, printer utilized, mechanical characteristics evaluated, and sample standards for those publications. Furthermore, a summary of 3D printed CFRC's mechanical properties along with their processing (printing), pre-/post-processing conditions are presented in Table 3. In comparison to CFRCs manufactured conventionally, printed CFRCs have inferior mechanical characteristics. The existence of flaws such as vacancies and poorer layer bonding are two primary reasons for this. As a result, several studies have attempted to enhance the mechanical characteristics of CFRCs by various methods such as impregnation, rolling, pressing, and heat treatment (see Fig. 11). In addition,

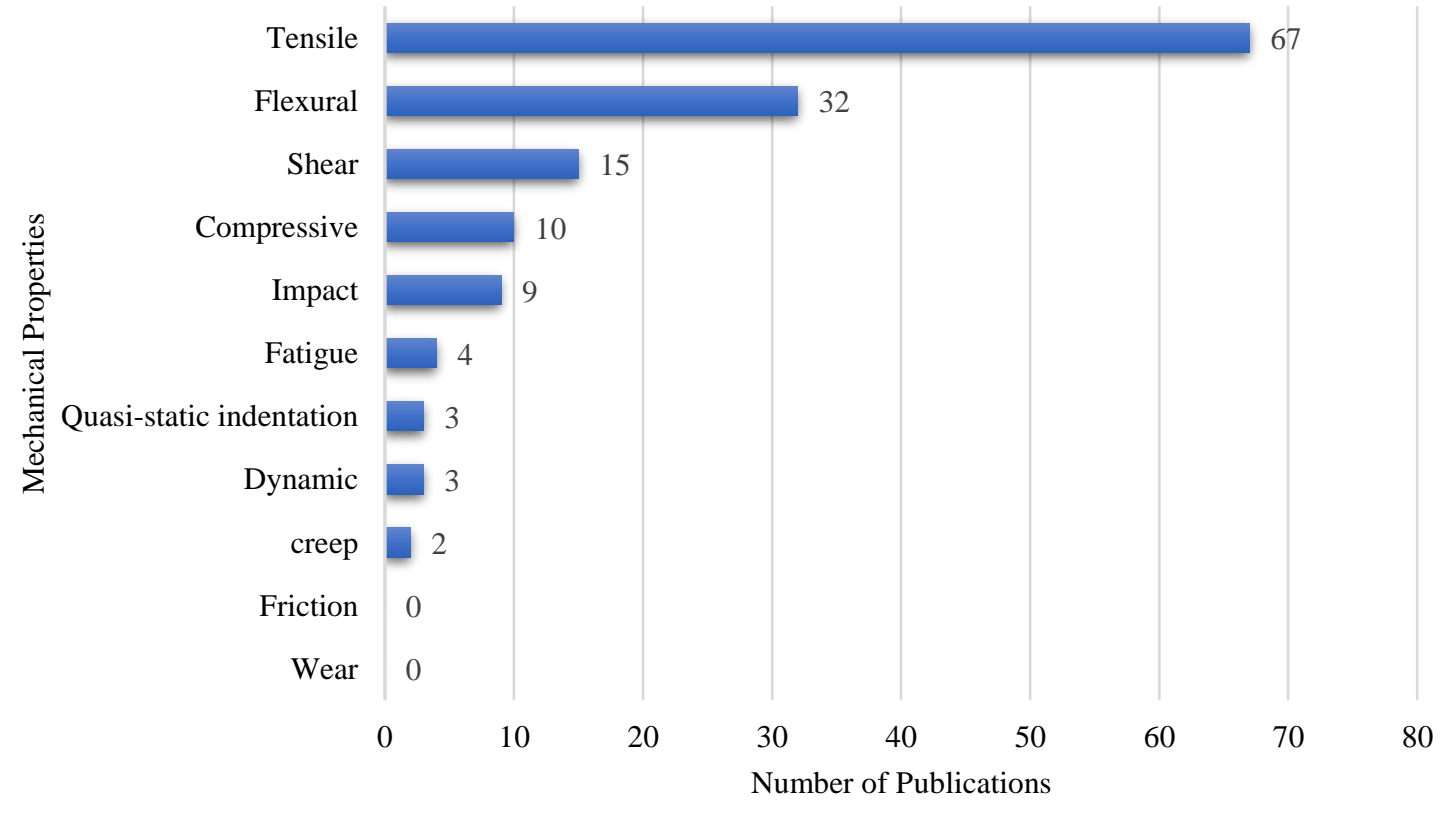

Fig. 6 shows the number of papers on mechanical properties, with tensile and flexural testing being the most popular among academics. As a result, just the tensile and flexural properties of the 3D printed CFRCs will be discussed. Another thing to note is that no papers have focused on the wear 


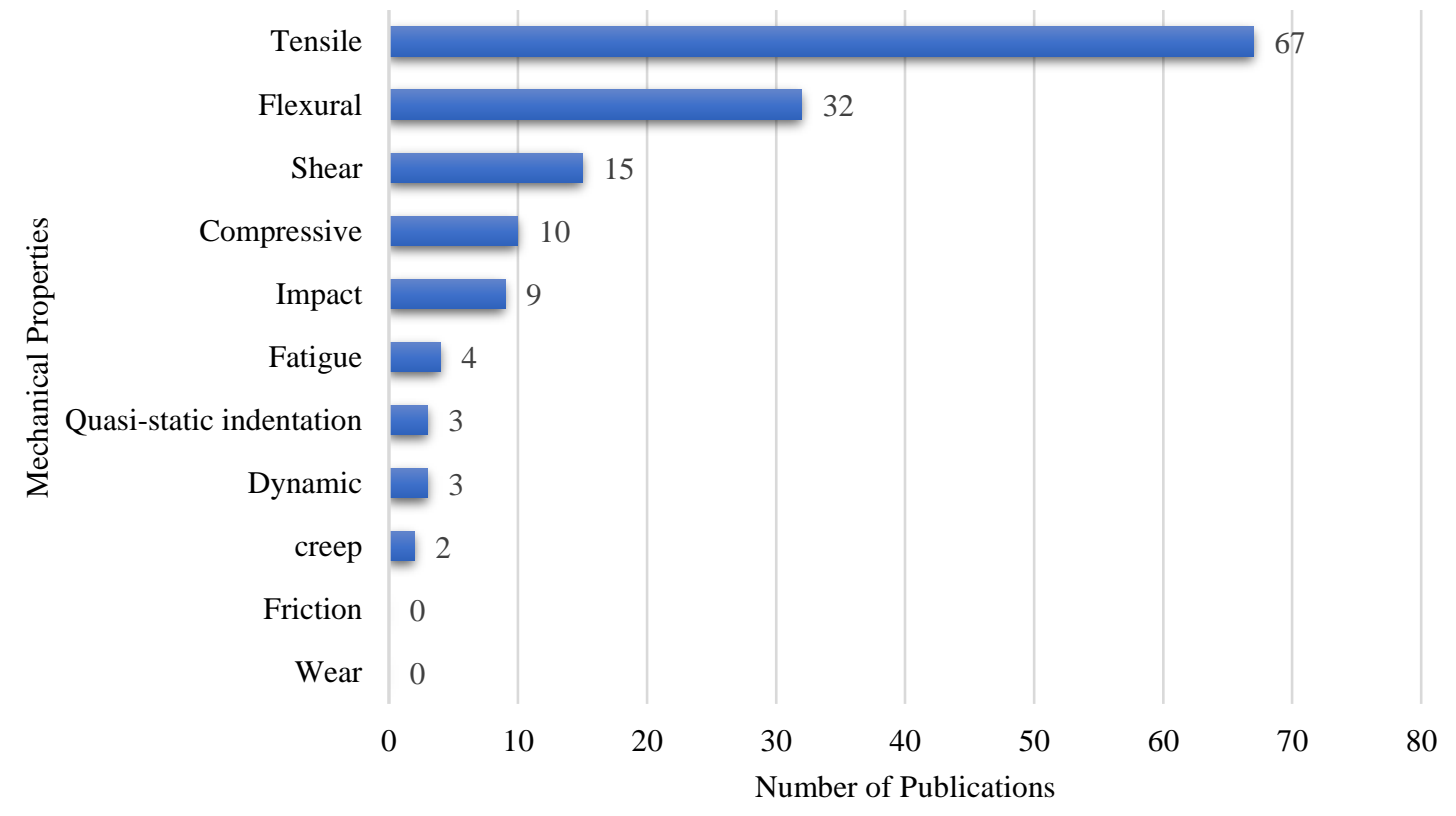

Fig. 6), which could be interesting subjects for future researches. In addition,

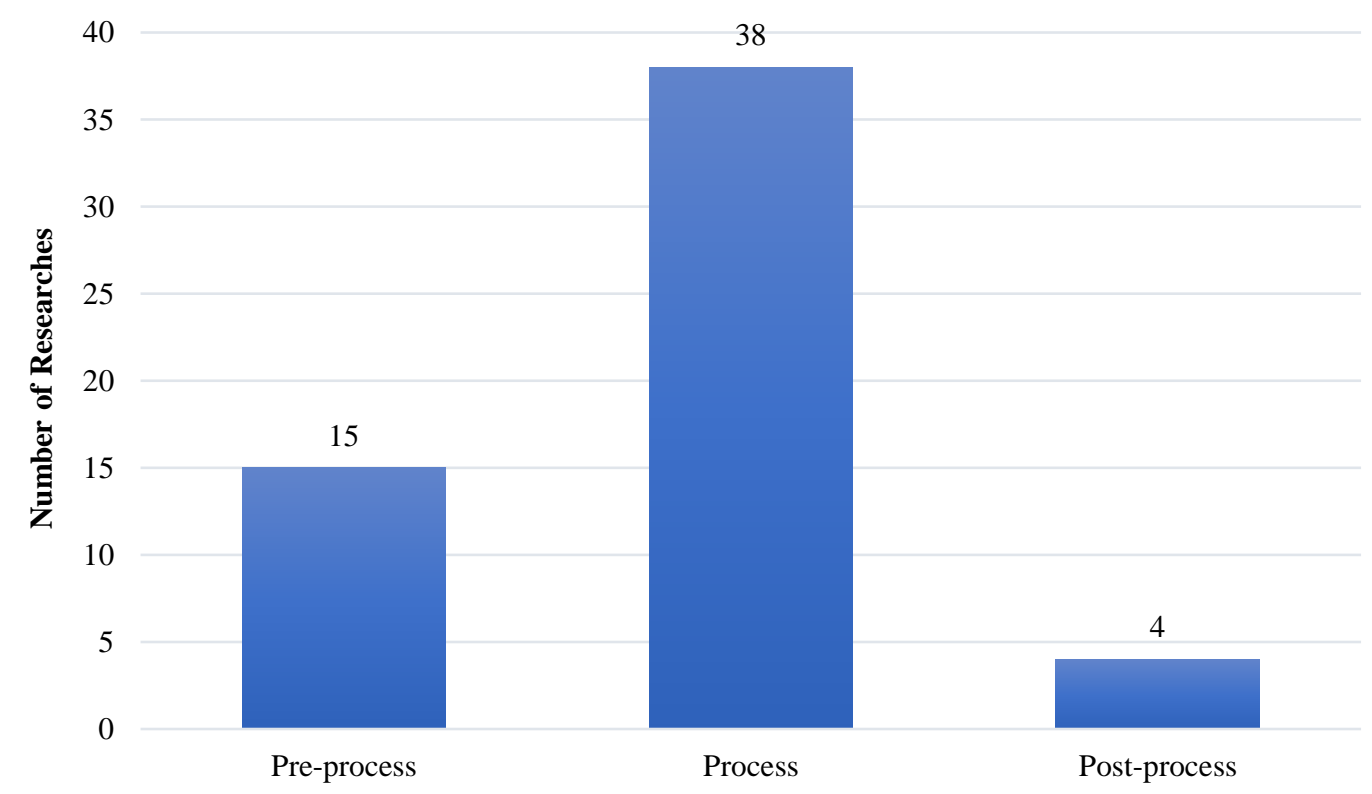

Fig. 7 divides CFRC research into three categories: pre-processing, processing, and postprocessing. As can be seen, the majority of the study focuses on the processing conditions, with 
just a few studies focusing on improving the mechanical characteristics of CFRC through postprocessing. As a result, it might be an intriguing topic for future research.

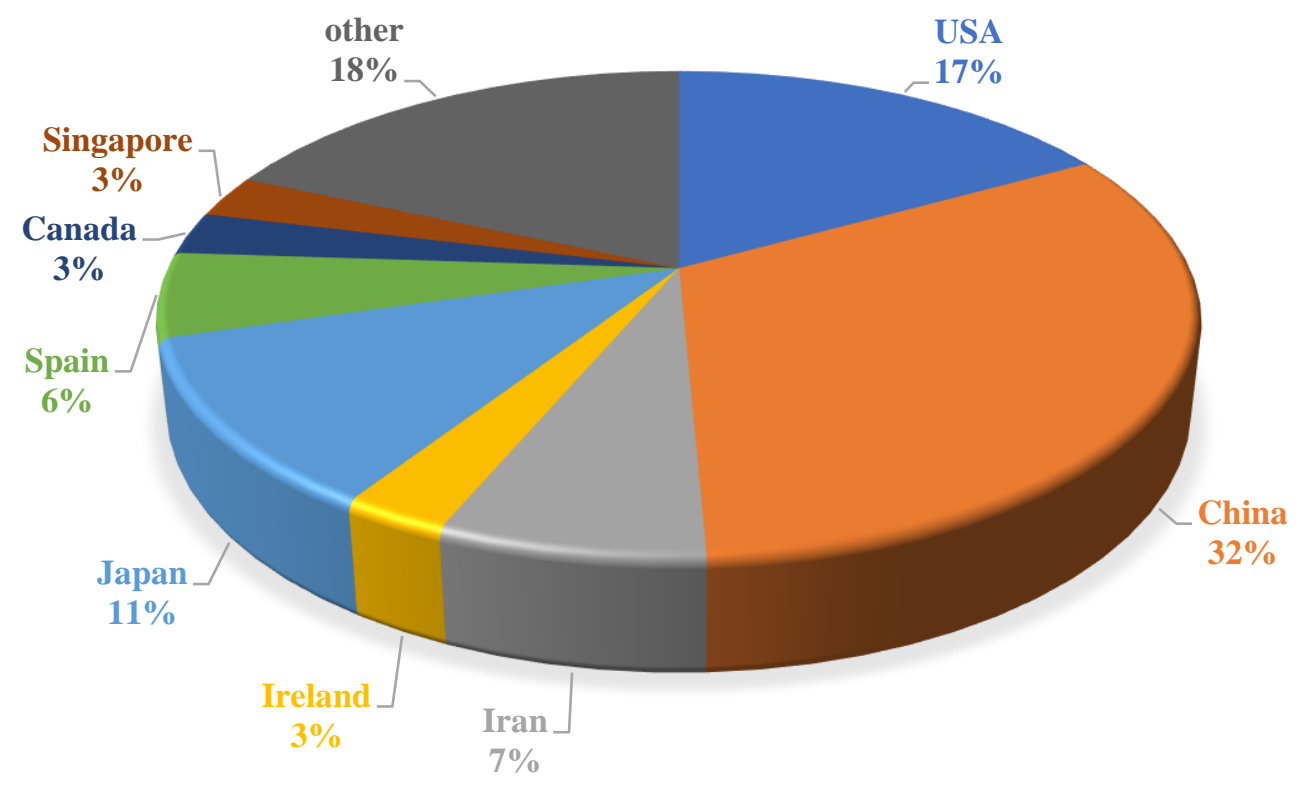

Fig. 8 shows a list of the nations where the studies were done. China, the United States, Japan, Iran, Spain, Ireland, Canada, and Singapore are the nations that have concentrated on CFRC 3D printing, according to this graph. Many businesses have succeeded in developing and manufacturing 3D printers, particularly FDM. Different varieties of these printers have been used to produce examples in the literature, however many publications have used Markforged's 


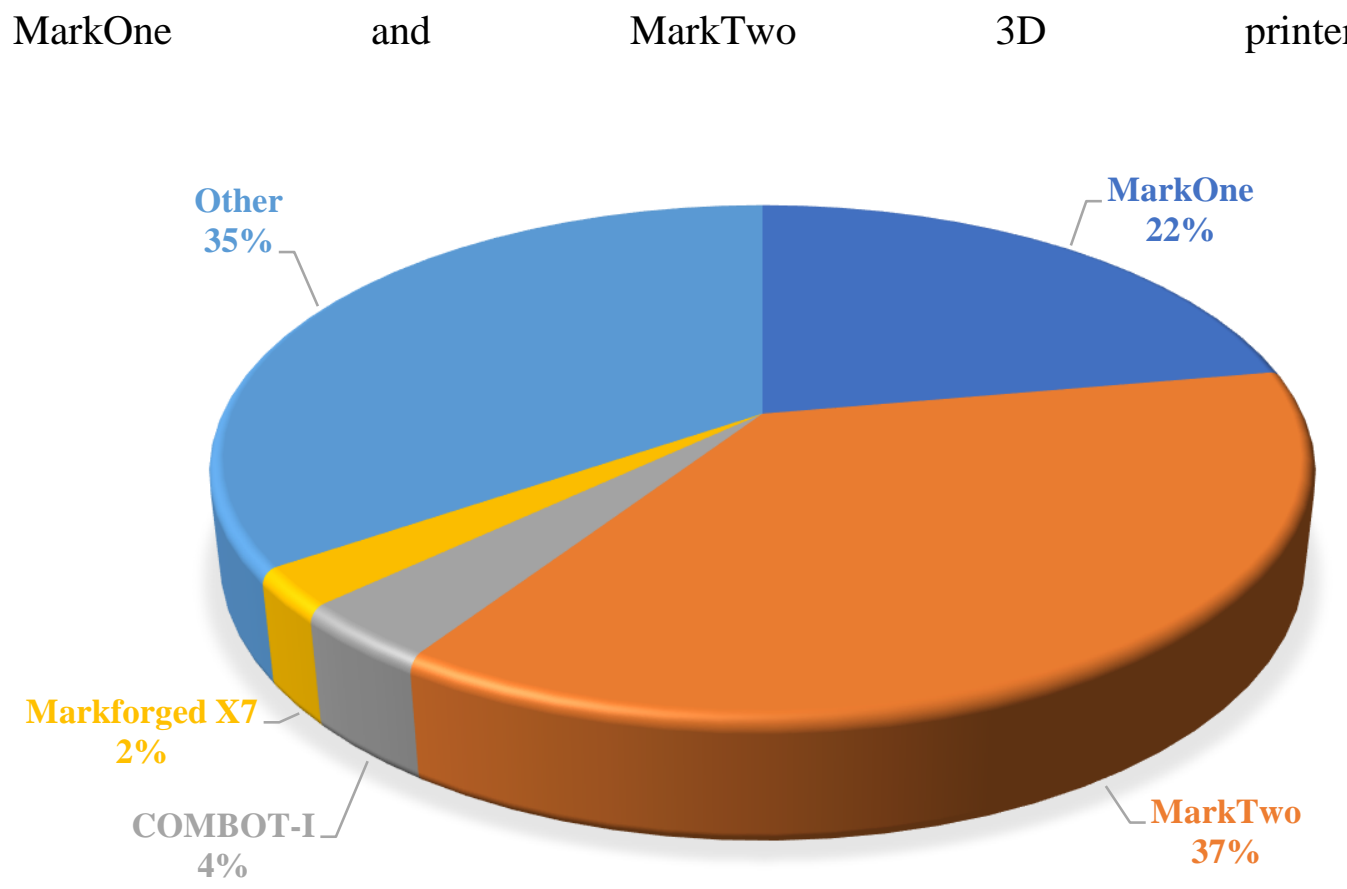

Fig. 9).

The number of publications published on the subject of CFRC 3D printing has been growing every year,

as

shown

in

30

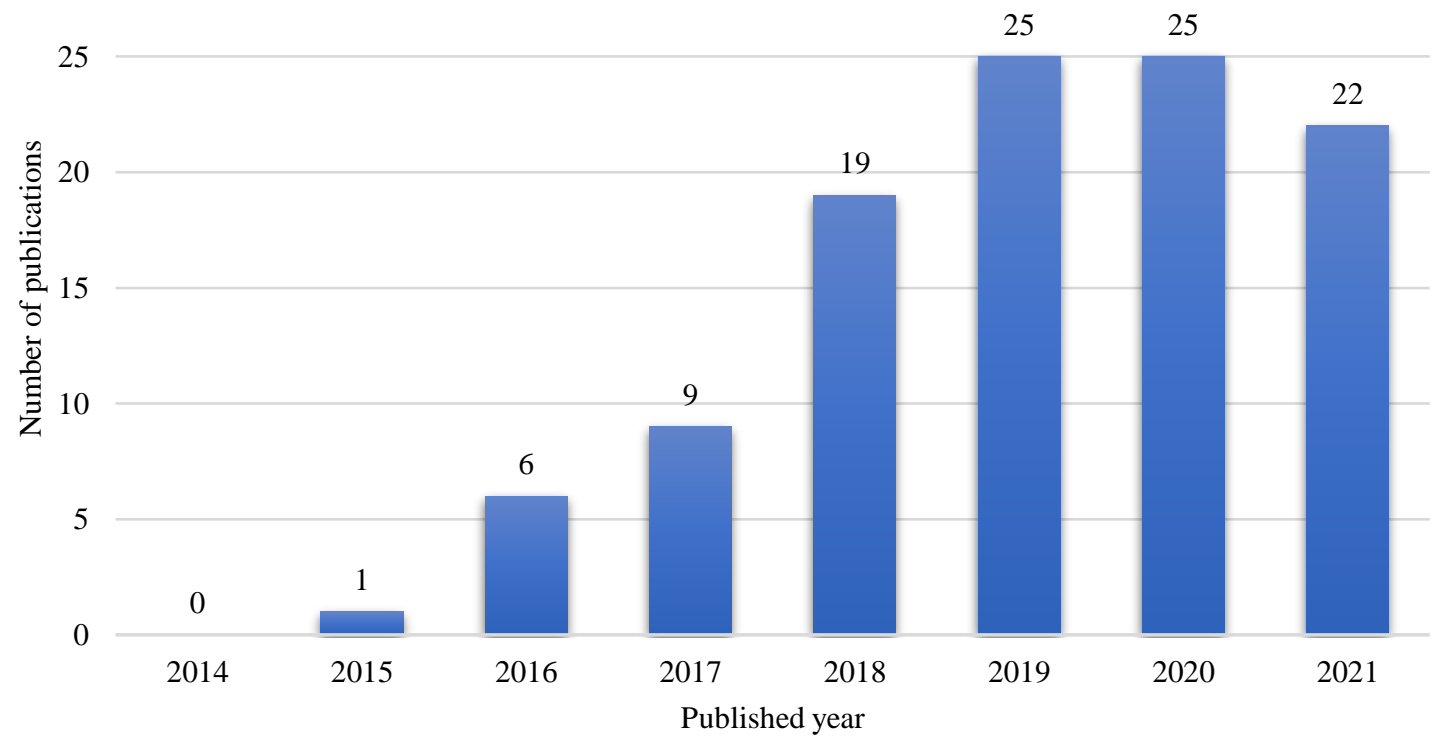


Fig. 10. In contrast to the trend, there was no rise in the number of papers published in 2020. This might be related to the coronavirus epidemic and most nations' quarantine policies. Given that this article is being written in the middle of 2021, a substantial number of articles have already been published in 2021, implying that by the end of 2021, the number of articles published will be significantly greater than in 2020 . 
Table 2. A summary of CFRC 3D printing research studies

\begin{tabular}{|c|c|c|c|c|c|c|c|}
\hline Author/Authors & $\begin{array}{c}\text { Published } \\
\text { Year }\end{array}$ & Research Object & Matrix Material & $\begin{array}{c}\text { Reinforcement } \\
\text { Material }\end{array}$ & 3D Printer & $\begin{array}{c}\text { Studied Mech. } \\
\text { Prop. }\end{array}$ & Specimen Design \\
\hline Akhoundi et al. [28] & 2020 & In-melt simultaneous impregnation & $\begin{array}{l}\text { PLA, PLA+, PLA- } \\
\text { wood, TPU, HDglass, } \\
\text { POM, PA+CF }\end{array}$ & GF & - & Tensile & ASTM D638, type IV \\
\hline Akhoundi et al. [17] & 2019 & Improving mechanical properties & PLA & GF & - & $\begin{array}{l}\text { Tensile, bulk } \\
\text { density }\end{array}$ & ASTM D638, type IV, ASTM D792 \\
\hline Al Abadi et al. [18] & 2018 & Evaluation of elastic properties & Nylon & $\mathrm{CF}, \mathrm{GF}, \mathrm{KF}$ & MarkOne & Tensile & ASTM D3039 \\
\hline Araya-Calvo et al. [29] & 2018 & Improving mechanical properties & Onyx & $\mathrm{CF}$ & MarkTwo & $\begin{array}{l}\text { Compressive, } \\
\text { Flexural }\end{array}$ & ASTM D695, ASTM D790 \\
\hline Babu et al. [30] & 2021 & $\begin{array}{l}\text { Influence of slicing parameters on surface } \\
\text { quality and mechanical properties }\end{array}$ & PLA & $\mathrm{CF}$ & $\begin{array}{l}\text { Raise 3D V2 } \\
\qquad \text { N2 }\end{array}$ & $\begin{array}{c}\text { Tensile, } \\
\text { Flexural, } \\
\text { Interlaminar } \\
\text { shear strength }\end{array}$ & $\begin{array}{l}\text { ASTM D638, ASTM D790, ASTM } \\
\text { D2344 }\end{array}$ \\
\hline Baumann et al. [31] & 2017 & $\begin{array}{c}\text { Investigation of a new approach for } \\
\text { additively manufactured continuous fiber- } \\
\text { reinforced polymers }\end{array}$ & ABS & $\mathrm{CF}, \mathrm{GF}$ & - & Tensile & ISO 527 \\
\hline Bettini et al. [32] & 2017 & Improving mechanical properties & PLA & Aramid & Blue Tek Strato & $\begin{array}{c}\text { Tensile, } \\
\text { Compressive }\end{array}$ & $\begin{array}{l}\text { for tensile tests } 250 \mathrm{mmx} 15 \mathrm{mmx} 0.95 \\
\mathrm{~mm} / \text { for compression tests } 60 \mathrm{~mm} \times 21.3 \\
\mathrm{mmx} 1.5 \mathrm{~mm}\end{array}$ \\
\hline Caminero et al. [33] & 2018 & Improving Impact damage resistance & Nylon & $\mathrm{CF}, \mathrm{GF}, \mathrm{KF}$ & MarkTwo & Impact & ASTM D6110 \\
\hline Cersoli et al. [34] & 2021 & Evaluation mechanical properties & PLA & KF & $\begin{array}{l}\text { MakerGear } \\
\text { M2 }\end{array}$ & $\begin{array}{l}\text { Tensile, } \\
\text { Flexural, }\end{array}$ & $\begin{array}{l}\text { ASTM D3039, ASTM D7264, } 100 \times x \\
100 \times 5.5\end{array}$ \\
\hline
\end{tabular}




\begin{tabular}{|c|c|c|c|c|c|c|c|}
\hline & & & & & & Impact & \\
\hline Chacon et al. [35] & 2019 & $\begin{array}{c}\text { Effect of process parameters on mechanical } \\
\text { properties }\end{array}$ & Nylon & $\mathrm{CF}, \mathrm{GF}, \mathrm{KF}$ & MarkTwo & $\begin{array}{l}\text { Tensile, } \\
\text { Flexural }\end{array}$ & ASTM D3039, ASTM D790 \\
\hline Chaudhry et al. [36] & 2019 & $\begin{array}{l}\text { Effect of CF on reinforcement of } \\
\text { thermoplastics using FDM and RSM }\end{array}$ & PLA & $\mathrm{CF}$ & ANET A-8M & $\begin{array}{l}\text { Tensile, } \\
\text { Flexural }\end{array}$ & $\begin{array}{l}\text { ASTM-D3039/D3039M, ASTM-D638, } \\
\text { ASTM-D790, ASTM-D7264/D7264M }\end{array}$ \\
\hline Chen et al. [37] & 2021 & Optimization of printing parameters & PLA & GF & - & $\begin{array}{c}\text { Tensile, } \\
\text { Flexural, } \\
\text { Impact, Short } \\
\text { beam shear }\end{array}$ & $\begin{array}{l}\text { GB/T1447-2005, GB/T 1449-2005, } \\
\text { GB/T 1451-2005, JC/T 773-2010 }\end{array}$ \\
\hline Chen et al. [38] & 2021 & $\begin{array}{c}\text { Evaluation of mechanical properties \& } \\
\text { fracture behavior }\end{array}$ & Onyx & $\mathrm{CF}$ & MarkTwo & Tensile & ASTM D638-14 Type IV \\
\hline Dickson et al. [39] & 2017 & $\begin{array}{c}\text { Influence of process parameters on } \\
\text { mechanical properties }\end{array}$ & Nylon & $\mathrm{CF}, \mathrm{GF}, \mathrm{KF}$ & MarkOne & $\begin{array}{l}\text { Tensile, } \\
\text { Flexural }\end{array}$ & ASTM D3039, ASTM D790 \\
\hline Dikshit et al. [40] & 2019 & Quasi-static indentation analysis & CR-WT & $\mathrm{KF}$ & $\begin{array}{c}\text { MarkOne, } \\
\text { InkJet }\end{array}$ & $\begin{array}{l}\text { Quasi-static } \\
\text { indentation }\end{array}$ & ASTM D6264 \\
\hline Dong et al. [41] & 2018 & Evaluating mechanical properties & Nylon & KF & MarkOne & Tensile & ASTM D3039 \\
\hline Dong et al. [42] & 2021 & $\begin{array}{l}\text { Mechanical properties and shape memory } \\
\text { effect of 4D printed cellular structure } \\
\text { composite }\end{array}$ & PLA & $\mathrm{KF}$ & $\begin{array}{l}\text { Createbot } \\
\text { MID250 }\end{array}$ & Tensile & ASTM D3039 \\
\hline Dong et al. [43] & 2020 & Diamond cellular structural & PLA & KF & $\begin{array}{l}\text { Createbot } \\
\text { MID250 }\end{array}$ & Tensile & ASTM D3039 \\
\hline Dugbenoo et al. [44] & 2018 & Enhanced bonding & Nylon & $\mathrm{CF}, \mathrm{GF}, \mathrm{KF}$ & MarkOne & $\begin{array}{l}\text { single-lap-joint } \\
\text { (SLJ) }\end{array}$ & ASTM D2093 \\
\hline
\end{tabular}




\begin{tabular}{|c|c|c|c|c|c|c|c|}
\hline Dutra et al. [45] & 2019 & $\begin{array}{l}\text { Mechanical characterization and asymptotic } \\
\text { homogenization }\end{array}$ & Nylon & $\mathrm{CF}$ & MarkOne & $\begin{array}{l}\text { Tensile, } \\
\text { Compression, } \\
\text { In-plane shear }\end{array}$ & $\begin{array}{l}\text { ASTM D3039, ASTM D638, ASTM } \\
\text { D6641, ASTM D3518 }\end{array}$ \\
\hline Fernandes et al. [46] & 2021 & Evaluation mechanical properties & Onyx & $\mathrm{CF}$ & MarkTwo & $\begin{array}{c}\text { Tensile, } \\
\text { Interlaminar } \\
\text { shear, Dynamic } \\
\text { mechanical } \\
\text { analysis }\end{array}$ & $\begin{array}{c}\text { ASTM D638 -14, ASTM } \\
\text { D3039/D3039M-17, ASTM- } \\
\text { D2344/D2344M, ASTM D4056-12 }\end{array}$ \\
\hline Ghebretinsae et al. [47] & 2019 & $\begin{array}{l}\text { Strength analysis with using experimental } \\
\text { and numerical methods }\end{array}$ & Onyx & $\mathrm{CF}$ & MarkTwo & $\begin{array}{l}\text { Tensile, } \\
\text { Flexural }\end{array}$ & ASTM D3039, ASTM D7264 \\
\hline Giannakis et al. [48] & 2019 & Static and fatigue properties & Nylon, PLA & $\mathrm{CF}$ & $\begin{array}{l}\text { BCN3D, } \\
\text { MarkTwo }\end{array}$ & $\begin{array}{l}\text { Tensile, } \\
\text { Fatigue }\end{array}$ & ASTM D3039, custom specimens \\
\hline Goh et al. [49] & 2018 & $\begin{array}{l}\text { Characterization of mechanical properties } \\
\text { and fracture mode }\end{array}$ & Nylon & $\mathrm{CF}$ & MarkOne & $\begin{array}{c}\text { Tensile, } \\
\text { Flexural, } \\
\text { Quasi-static } \\
\text { indentation }\end{array}$ & $\begin{array}{c}\text { ASTM D3039, ASTM D790, ASTM } \\
\text { D6264 }\end{array}$ \\
\hline $\begin{array}{l}\text { Gonzalez-Estrada et al. } \\
\text { [50] }\end{array}$ & 2018 & Evaluation mechanical properties & Nylon & $\mathrm{CF}, \mathrm{GF}$ & MarkTwo & Tensile & ASTM D638, type IV \\
\hline Hao et al. [51] & 2018 & Preparation and characterization & Epoxy resin & $\mathrm{CF}$ & - & $\begin{array}{l}\text { Tensile, } \\
\text { Flexural }\end{array}$ & ISO 527, ISO 178 \\
\hline Hedayati et al. [52] & 2020 & $\begin{array}{l}\text { Study on mechanical and cell viability } \\
\text { properties }\end{array}$ & PCL & PGA suture & - & Tensile & ASTM D638, ASTM D2256 \\
\hline Hetrick et al. [54] & 2021 & Charpy impact energy absorption & Onyx & KF & MarkTwo & Charpy impact & ASTM D6110 \\
\hline
\end{tabular}




\begin{tabular}{|c|c|c|c|c|c|c|c|}
\hline Hou et al. [55] & 2020 & $\begin{array}{l}\text { A constitutive model for } 3 \mathrm{~d} \text { printed CFRC } \\
\text { structures with variable fiber content }\end{array}$ & PLA & KF & COMBOT-I & $\begin{array}{l}\text { Tensile, } \\
\text { Compressive, } \\
\text { In-plane shear }\end{array}$ & $\begin{array}{c}\text { GB/T 3354-2014, GB/T 1448-2005, } \\
\text { GB/T 3355-2014 }\end{array}$ \\
\hline Hu et al. [56] & 2018 & $\begin{array}{c}\text { Manufacturing and 3D printing of prepreg } \\
\text { filament }\end{array}$ & PLA & $\mathrm{CF}$ & Mendel & Flexural & $60 \mathrm{~mm} \times 11 \mathrm{~mm} \times 3$ layer thickness \\
\hline Hu et al. [57] & 2021 & Fiber damage during 3D printing & - & CF-PLA & MarkTwo & $\begin{array}{c}\text { Tensile, } \\
\text { Compressive }\end{array}$ & $\begin{array}{l}200 \mathrm{~mm} \times 150 \mathrm{~mm} \times 2 \mathrm{~mm}, 12 \mathrm{~mm} \times \\
12 \mathrm{~mm} \times 30 \mathrm{~mm}\end{array}$ \\
\hline Ibrahim et al. [58] & 2020 & Thermal conductivity & Nylon & $\mathrm{CF}$ & MarkOne & $\begin{array}{l}\text { Effective } \\
\text { thermal } \\
\text { conductivity }\end{array}$ & Variable \\
\hline Imeri et al. [59] & 2018 & Fatigue analysis & Nylon & $\mathrm{CF}, \mathrm{GF}, \mathrm{KF}$ & MarkTwo & Fatigue & ASTM E606M \\
\hline Ipekci et al. [60] & 2021 & $\begin{array}{l}\text { Experimental and statistical analysis of } \\
\text { robotic 3D printing }\end{array}$ & photopolymer & GF & $\begin{array}{l}\text { NACHI brand } \\
\text { MZ07L }\end{array}$ & $\begin{array}{l}\text { Tensile, } \\
\text { Flexural, izod } \\
\text { impact }\end{array}$ & $\begin{array}{c}\text { ASTM D3039, ASTM D7264, ASTM } \\
\text { D256 }\end{array}$ \\
\hline Iraqi et al. [61] & 2019 & Evaluate the mechanical properties & Onyx & $\mathrm{CF}$ & MarkTwo & $\begin{array}{l}\text { Tensile, In- } \\
\text { plane shear, } \\
\text { Interlaminar } \\
\text { shear } \\
\text { resistance }\end{array}$ & $\begin{array}{l}\text { ASTM D3039, ASTM D518, ASTM } \\
\text { D2344 }\end{array}$ \\
\hline Ishii et al. [62] & 2018 & Bending fracture rule & Nylon & $\mathrm{CF}$ & MarkTwo & $\begin{array}{l}\text { Bending } \\
\text { fracture }\end{array}$ & Curved CF \\
\hline Jahangir et al. [63] & 2019 & Porosity reduction & Polycarbonate (PC) & $\mathrm{CF}$ & $400 \mathrm{mc}$ & Tensile, Warp & ASTM D638 \\
\hline Justo et al. [64] & 2018 & $\begin{array}{l}\text { Mechanical characterization of long fiber } \\
\text { composites }\end{array}$ & Nylon & $\mathrm{CF}, \mathrm{GF}$ & MarkOne & $\begin{array}{l}\text { Tensile, } \\
\text { Compressive, } \\
\text { In-plane shear }\end{array}$ & $\begin{array}{l}\text { ASTM D3039, ASTM D695-02a, } \\
\text { ASTM D3518 }\end{array}$ \\
\hline
\end{tabular}




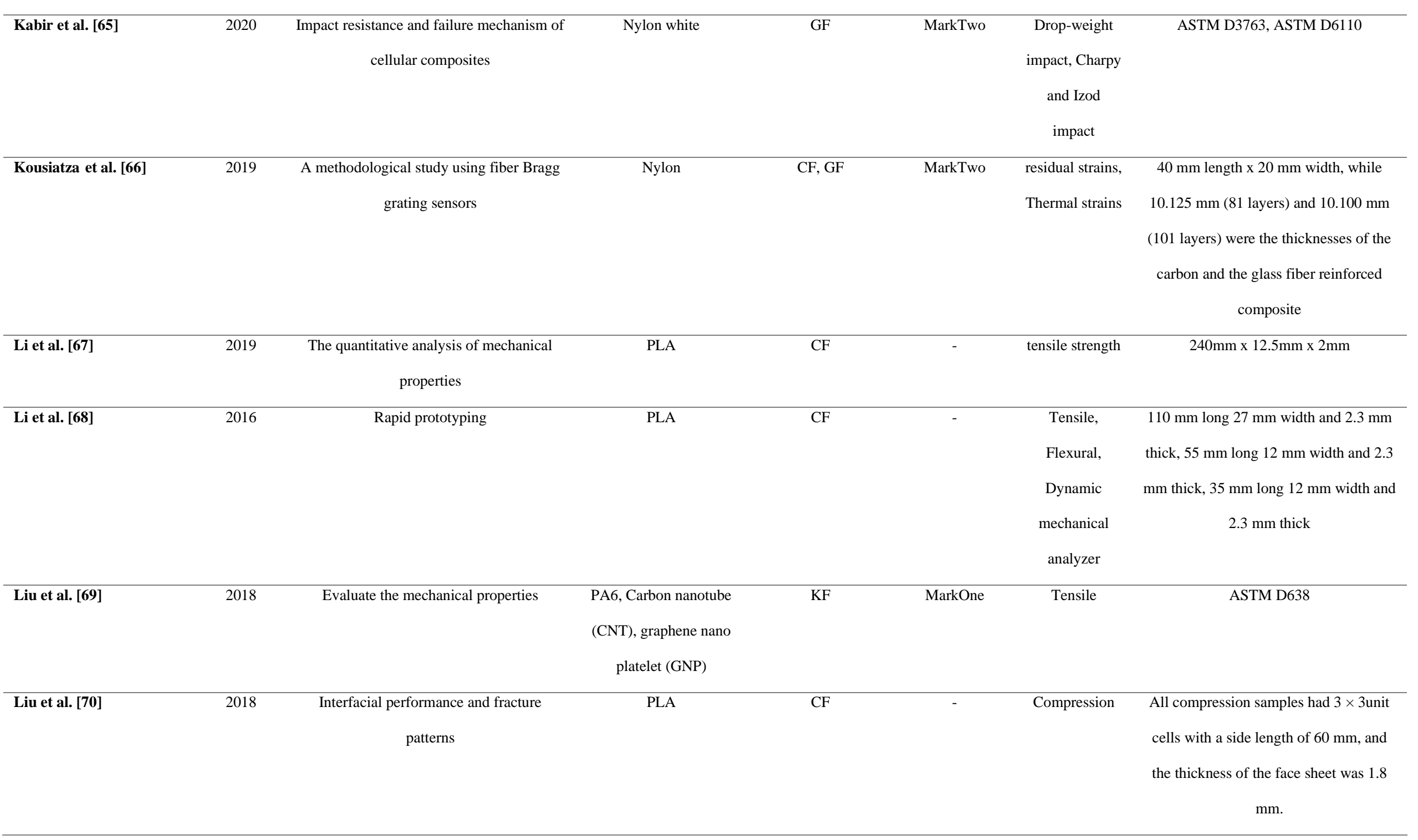




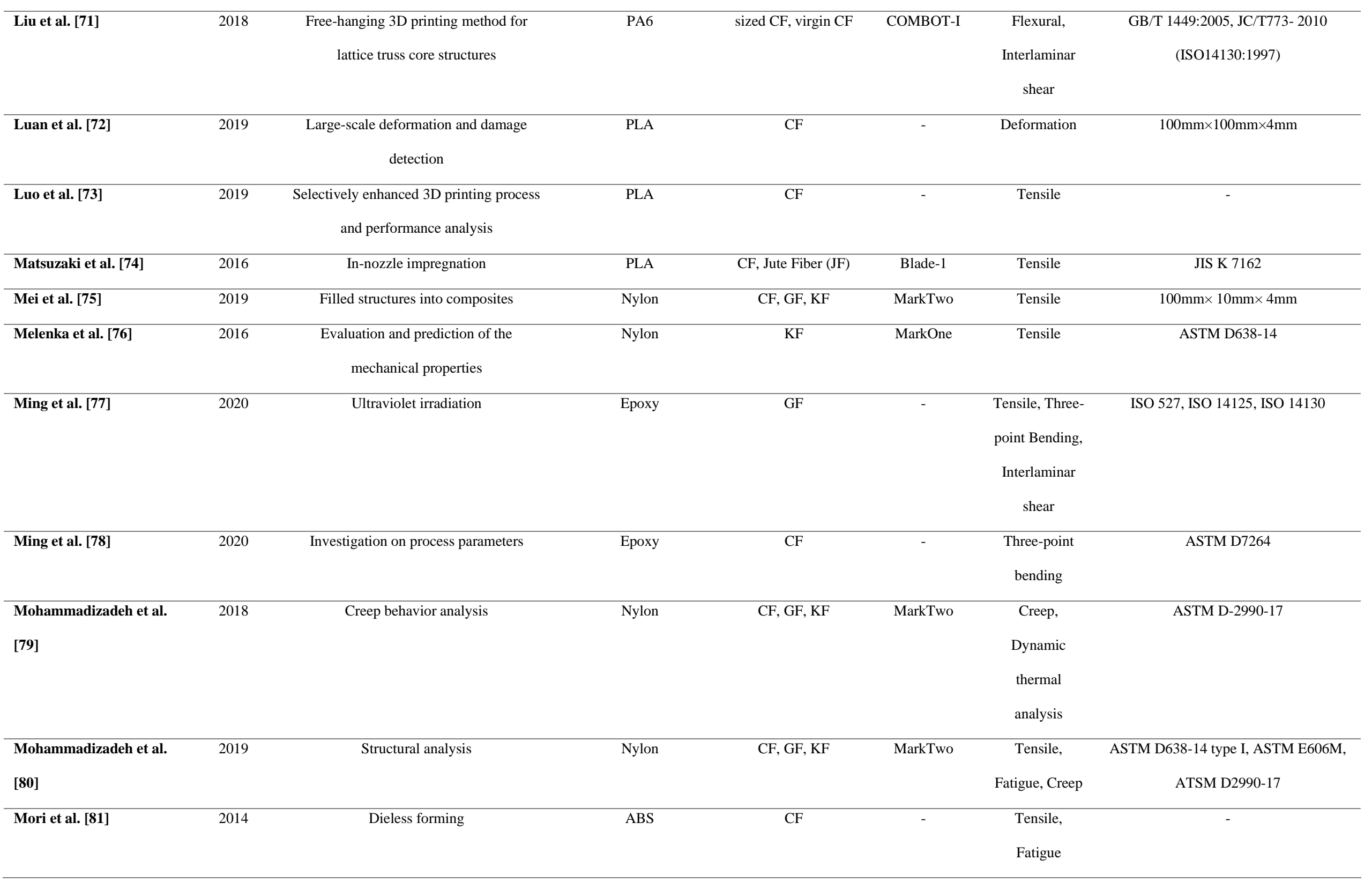




\begin{tabular}{|c|c|c|c|c|c|c|c|}
\hline Mosleh et al. [82] & 2021 & Determining process-window & ABS & $\mathrm{CF}$ & Sizan Lite & $\begin{array}{l}\text { Tensile, } \\
\text { Flexural, } \\
\text { interlaminar } \\
\text { shear }\end{array}$ & $\begin{array}{c}\text { ASTM D3039, ASTM D790, ASTM } \\
\text { D2344 }\end{array}$ \\
\hline Nabipour et al. [83] & 202 & Process parameters & ABS & Cu powder & Quantum & tensile strength & ASTM-D638 Type IV \\
\hline Naranjo-Lozada et al. [84] & 2019 & Mechanical properties and failure behavior & Nylon & $\mathrm{CF}$ & MarkTwo & Tensile & ASTM D638 \\
\hline O'Connor et al. [85] & 2019 & Low-pressure AM & Nylon & $\mathrm{CF}, \mathrm{GF}, \mathrm{KF}$ & MarkOne & $\begin{array}{l}\text { Interlaminar } \\
\text { shear }\end{array}$ & ASTM D2344 \\
\hline Oztan et al. [86] & 2019 & Microstructure and mechanical properties & PLA, Nylon & $\mathrm{CF}, \mathrm{KF}$ & $\begin{array}{l}\text { MarkOne, } \\
\text { Ultimaker } 2\end{array}$ & Tensile & ASTM D638, ASTM D3039 \\
\hline Peng et al. [87] & 2020 & $\begin{array}{l}\text { Tailorable rigidity and energy-absorption } \\
\text { capability }\end{array}$ & PLA & $\mathrm{CF}$ & Mark X & Flexural & $90 \mathrm{~mm} \times 6 \mathrm{~mm} \times 6 \mathrm{~mm}$ \\
\hline Prajapati et al. [88] & 2021 & $\begin{array}{l}\text { Evaluating mechanical, thermal, flame- } \\
\text { retardant properties }\end{array}$ & Onyx & $\begin{array}{l}\text { GF, high strength } \\
\text { high temperature } \\
\text { (HSHT) GF }\end{array}$ & MarkTwo & Tensile, Impact & ASTM D638, ASTM D256 \\
\hline Prajapati et al. [89] & 2021 & $\begin{array}{l}\text { Effect of fiber volume fraction on the } \\
\text { impact strength }\end{array}$ & Onyx & GF & MarkTwo & Impact strength & ASTM D256 \\
\hline Prajapati et al. [90] & 2021 & Open hole tensile strength & Onyx & HSHT GF & MarkTwo & Tensile & ASTM D5766 \\
\hline Pyl et al. [91] & 2018 & Design of test specimens & Nylon & $\mathrm{CF}$ & MarkTwo & $\begin{array}{l}\text { Tensile, In- } \\
\text { plane shear }\end{array}$ & $\begin{array}{l}\text { ASTM D638-14 Type I, ASTM D638 } \\
\text { type IV, ASTM D638 type IV modified, } \\
\text { ASTM D3039 }\end{array}$ \\
\hline Qiao et al. [92] & 2019 & Ultrasonic modification & PLA & $\mathrm{CF}$ & MarkOne & $\begin{array}{l}\text { Tensile, } \\
\text { Flexural }\end{array}$ & ASTM D3039-14, ASTM D7264-07 \\
\hline Quan et al. [93] & 2020 & Auxetic honeycomb structures & PLA & $\mathrm{KF}$ & - & $\begin{array}{c}\text { Tensile, } \\
\text { Compression }\end{array}$ & - \\
\hline
\end{tabular}




\begin{tabular}{|c|c|c|c|c|c|c|c|}
\hline Sanei et al. [94] & 2020 & $\begin{array}{c}\text { Effects of stress concentration on the } \\
\text { mechanical properties }\end{array}$ & Onyx & $\mathrm{CF}$ & MarkTwo & Tensile & ASTM D3039 \\
\hline Sanei et al. [95] & 2019 & Evaluating mechanical properties & Onyx & $\mathrm{CF}$ & $\begin{array}{c}\text { Markedforged } \\
\text { X7 }\end{array}$ & Tensile & ASTM D3039 \\
\hline Sarvestani et al. [96] & 2017 & Evaluating mechanical properties & Nylon & $\mathrm{CF}$ & MarkTwo & Tensile & $\begin{array}{l}\text { ASTM D638-14 Type I, ASTM D638- } \\
14 \text { Type IV, ASTM D3039 }\end{array}$ \\
\hline Shang et al. [26] & 2020 & Inter-line bonding & PLA & $\mathrm{CF}$ & Fibertech & Tensile & GB/T3354-2014 \\
\hline Shi et al. [97] & 2021 & Evaluating mechanical properties & Nylon & $\mathrm{KF}$ & MarkTwo & Tensile & ASTM D3039 \\
\hline Shiratori et al. [98] & 2021 & $\begin{array}{l}\text { Evaluating the strength of curved sections } \\
\text { of 3D printed specimens }\end{array}$ & Nylon & $\mathrm{CF}$ & MarkTwo & $\begin{array}{c}\text { Tensile, } \\
\text { Compressive, } \\
\text { Three-point } \\
\text { bending }\end{array}$ & $\begin{array}{l}\text { ASTM D 6415, L-shape specimen, Flat } \\
\text { specimens }\end{array}$ \\
\hline Sugiyama et al. [99] & 2020 & Optimized composites & Nylon & $\mathrm{CF}$ & BS01+ & $\begin{array}{l}\text { Laminate } \\
\text { Bearing }\end{array}$ & ASTM D5961 \\
\hline Sugiyama et al. [100] & 2018 & Sandwich structures & - & $\mathrm{CF}$ & BS01+ & $\begin{array}{l}\text { Tensile, Three- } \\
\text { point bending }\end{array}$ & JIS K 7165: 2008, JIS K 7017: 1999 \\
\hline Tian et al. [101] & 2017 & Recycling and remanufacturing & PLA & $\mathrm{CF}$ & - & $\begin{array}{c}\text { Tensile, } \\
\text { Flexural, } \\
\text { Interlaminar } \\
\text { shear, Impact }\end{array}$ & $\begin{array}{c}\text { GB/T 1447:2005, GB/T 1449:2005, } \\
\text { JC/T773- } 2010 \text { (ISO14130:1997), GB/T } \\
\text { 1043:1993 }\end{array}$ \\
\hline Tian et al. [102] & 2016 & Interface and performance & PLA & $\mathrm{CF}$ & - & Flexural & ISO 14125:1998 \\
\hline Todoroki et al. [103] & 2020 & Evaluating mechanical properties & Nylon & $\mathrm{CF}$ & MarkTwo & Tensile & $\begin{array}{c}\text { Custom specimens with no surface } \\
\text { layers }\end{array}$ \\
\hline
\end{tabular}




\begin{tabular}{|c|c|c|c|c|c|c|c|}
\hline Touchard et al. [104] & 2021 & Interfacial adhesion quality & PA6 & $\mathrm{CF}$ & MarkTwo & $\begin{array}{l}\text { Tensile, } \\
\text { Interlaminar } \\
\text { shear }\end{array}$ & - \\
\hline Ueda et al. [27] & 2020 & 3D compaction printing & Nylon & $\mathrm{CF}$ & MarkTwo & $\begin{array}{l}\text { Tensile, Three- } \\
\text { point bending }\end{array}$ & ISO 527-5, ISO 14125 \\
\hline Van der klift et al. [105] & 2016 & Evaluating mechanical properties & Nylon & $\mathrm{CF}$ & MarkOne & Tensile & JIS K 7073 \\
\hline Vaneker et al. [106] & 2017 & $\begin{array}{l}\text { Material extrusion of continuous fiber } \\
\text { reinforced plastics using commingled yarn }\end{array}$ & polypropylene (PP) & GF & MarkOne & $\begin{array}{l}\text { Flexural } \\
\text { modulus }\end{array}$ & ISO14125 \\
\hline Wang et al. [107] & 2021 & $\begin{array}{l}\text { Simultaneous reinforcement of both rigidity } \\
\text { and energy absorption }\end{array}$ & Onyx & $\mathrm{CF}, \mathrm{KF}$ & Mark7 & $\begin{array}{l}\text { quasi-static } \\
\text { indentation }\end{array}$ & $50.0 \mathrm{~mm} \times 50.0 \mathrm{~mm} \times 1.0 \mathrm{~mm}$ \\
\hline Wang et al. [108] & 2020 & $\begin{array}{l}\text { Evaluating Mechanical Properties and } \\
\text { microstructures }\end{array}$ & $\mathrm{Pb} 50 \mathrm{Sn} 50$ & $\mathrm{Cu}-\mathrm{CF}$ & COMBOT-I & Tensile & ASTM D3552-96 \\
\hline Wang et al. [109] & 2021 & $\begin{array}{c}\text { Process parameters and mechanical } \\
\text { properties }\end{array}$ & PLA & GF & - & $\begin{array}{l}\text { Tensile, } \\
\text { Flexural }\end{array}$ & ISO 14125: 1998 \\
\hline Yang et al. [110] & 2017 & Mechanism and performance & ABS & $\mathrm{CF}$ & - & $\begin{array}{l}\text { Tensile, Three- } \\
\text { point bending, } \\
\text { Interlaminar } \\
\text { shear test }\end{array}$ & $\begin{array}{l}\text { ISO 527:1997, ISO 14125:1998, ISO } \\
14130: 1998\end{array}$ \\
\hline Yao et al. [111] & 2017 & Evaluating mechanical properties & PLA & $\mathrm{CF}$ & $\begin{array}{l}\text { Kossel Rostock } \\
\text { Delta D-force }\end{array}$ & $\begin{array}{l}\text { Tensile, Three- } \\
\text { point bending }\end{array}$ & ISO 527-4:1997, ISO 14125: 1998 \\
\hline Yin et al. [112] & 2019 & Evaluating mechanical properties & PLA & $\mathrm{CF}$ & COMBOT-I & $\begin{array}{c}\text { Tensile, } \\
\text { Flexural, } \\
\text { Shielding } \\
\text { Effectiveness }\end{array}$ & $\begin{array}{l}\text { The diameters of the brim and crown of } \\
\text { the shell were } 130 \text { and } 100 \mathrm{~mm} \text {, } \\
\text { respectively. The total height was } 15 \\
\mathrm{~mm}\end{array}$ \\
\hline
\end{tabular}




\begin{tabular}{|c|c|c|c|c|c|c|c|}
\hline Yu et al. [113] & 2019 & Mechanical properties behaviors & Onyx & $\mathrm{CF}$ & MarkOne & $\begin{array}{l}\text { Tensile, } \\
\text { Flexural }\end{array}$ & $\begin{array}{l}92.5 \mathrm{~mm} \text { in length and } 3.25 \mathrm{~mm} \text { in height, } \\
\text { ASTM D6272-17 }\end{array}$ \\
\hline Zeng et al. [114] & 2021 & $\begin{array}{c}\text { Bending performance and failure behavior } \\
\text { of with shape memory capability }\end{array}$ & PLA & $\mathrm{CF}$ & - & $\begin{array}{l}\text { Three-point } \\
\text { bending }\end{array}$ & ASTM D7249/D7249M \\
\hline Zhang et al. [115] & 2020 & $\begin{array}{c}\text { Performance of 3D-Printed Continuous- } \\
\text { Carbon-Fiber-Reinforced Plastics with } \\
\text { Pressure }\end{array}$ & PLA & $\mathrm{CF}$ & - & $\begin{array}{l}\text { Tensile, Three- } \\
\text { point bending }\end{array}$ & GB/T1040.1-2006, GB/T449:2005 \\
\hline Zhang et al. [116] & 2021 & $\begin{array}{c}\text { Prediction of deformation and failure } \\
\text { behavior }\end{array}$ & PLA & $\mathrm{CF}$ & - & $\begin{array}{c}\text { Tensile, } \\
\text { Compressive, } \\
\text { In-plane shear, } \\
\text { Interlaminar } \\
\text { shear }\end{array}$ & $\begin{array}{l}\text { GB/T 1447-2005, ASTM-D6641, } \\
\text { ASTM-D3518, JC/T 773-2010 }\end{array}$ \\
\hline
\end{tabular}


Table 3. Summary of 3D printed CFRC's mechanical properties and their processing, pre-/postprocessing conditions

\begin{tabular}{|c|c|c|c|c|c|c|c|c|c|c|}
\hline Authors & $\begin{array}{c}\text { Matrix } \\
\text { Material }\end{array}$ & $\begin{array}{c}\text { Reinforcement } \\
\text { Material }\end{array}$ & $\begin{array}{c}\text { Fiber } \\
\text { volume } \\
\text { percentage }\end{array}$ & $\begin{array}{c}\text { Tensile } \\
\text { strength } \\
\text { (MPa) }\end{array}$ & $\begin{array}{c}\text { Tensile } \\
\text { modulus } \\
\text { (GPa) }\end{array}$ & $\begin{array}{c}\text { Flexural } \\
\text { strength } \\
\text { (MPa) }\end{array}$ & $\begin{array}{c}\text { Flexural } \\
\text { modulus } \\
\text { (GPa) }\end{array}$ & Pre-process & Process & $\begin{array}{l}\text { Post- } \\
\text { process }\end{array}$ \\
\hline Ueda et al. [27] & Nylon & $\mathrm{CF}$ & 35 & 1031 & 71.2 & 945 & 65.7 & - & $\begin{array}{c}\text { Hot- } \\
\text { compaction } \\
\text { roller }\end{array}$ & Hot-press \\
\hline Giannakis et al. [48] & Nylon, PLA & $\mathrm{CF}$ & - & 923 & 52.85509 & - & - & - & - & - \\
\hline Ming et al. [78] & Epoxy & $\mathrm{CF}$ & 58 & - & - & 952.89 & 74.05 & Impregnation & $\begin{array}{c}\text { Printing } \\
\text { speed, printing } \\
\text { space, printing } \\
\text { thickness }\end{array}$ & Curing \\
\hline Hu et al. [56] & PLA & $\mathrm{CF}$ & 25 & - & - & 610.092 & 40.13 & Impregnation & $\begin{array}{c}\text { Printing } \\
\text { speed, layer } \\
\text { thickness, } \\
\text { printing } \\
\text { temperature }\end{array}$ & - \\
\hline Liu et al. [71] & PA6 & $\begin{array}{l}\text { Sized } \mathrm{CF} \text {, } \\
\text { virgin } \mathrm{CF}\end{array}$ & 44.1 & - & - & 565.8 & 62.1 & Impregnation & $\begin{array}{l}\text { hatch spacing, } \\
\text { layer } \\
\text { thickness, feed } \\
\text { rate of } \\
\text { filament }\end{array}$ & - \\
\hline Hao et al. [51] & Epoxy resin & $\mathrm{CF}$ & - & 792.8 & 161.4 & 202 & 143.9 & - & Printing path, & Curing \\
\hline Iraqi et al. [61] & Onyx & $\mathrm{CF}$ & 27 & 779.8 & 60.9 & - & - & - & $\begin{array}{l}\text { Orthotropic } \\
\text { directions }\end{array}$ & - \\
\hline Hou et al. [55] & PLA & $\mathrm{KF}$ & 50 & 742.6 & 41.3 & - & - & - & Fiber contents & - \\
\hline Pyl et al. [91] & Nylon & $\mathrm{CF}$ & 27 & 719 & 58.07 & - & - & - & Fiber direction & - \\
\hline Justo et al. [64] & Nylon & $\begin{array}{l}\text { CF } \\
\text { GF }\end{array}$ & 40 & $\begin{array}{l}701.41 \\
574.58\end{array}$ & $\begin{array}{l}68.08 \\
25.86\end{array}$ & - & - & - & $\begin{array}{l}\text { The direction } \\
\text { of the fiber }\end{array}$ & - \\
\hline
\end{tabular}




\begin{tabular}{|c|c|c|c|c|c|c|c|c|c|c|}
\hline Todoroki et al. [103] & Nylon & $\mathrm{CF}$ & 30 & 701 & 60.9 & - & - & - & $\begin{array}{l}\text { Lay-up } \\
\text { direction }\end{array}$ & - \\
\hline Zhang et al. [115] & PLA & $\mathrm{CF}$ & 10.30 & 644.8 & - & 401.24 & - & - & Pressure roller & - \\
\hline Shiratori et al. [98] & Nylon & $\mathrm{CF}$ & 30 & 610.8 & - & - & - & - & - & - \\
\hline Goh et al. [49] & Nylon & $\mathrm{CF}$ & 41 & 600 & 13 & 430 & 38.1 & Impregnation & - & - \\
\hline $\begin{array}{c}\text { Ghebretinsae et al. } \\
\qquad[47]\end{array}$ & Onyx & $\mathrm{CF}$ & 42 & 559.9 & ro & $27 \cdot / N$ & $19 / 4$ & - & & - \\
\hline $\begin{array}{c}\text { Van Der Klift et al. } \\
{[105]}\end{array}$ & Nylon & $\mathrm{CF}$ & 34.5 & 520 & 35.7 & - & - & - & - & - \\
\hline Dutra et al. [45] & Nylon & $\mathrm{CF}$ & 32.8 & 493.9 & 45.2 & - & - & - & - & - \\
\hline Akhoundi et al. [17] & PLA & GF & 49.3 & 479 & 29.4 & - & - & Impregnation & $\begin{array}{c}\text { Fiber-volume } \\
\text { percentage }\end{array}$ & - \\
\hline \multirow[t]{2}{*}{ Chacon et al. [35] } & Nylon & $\begin{array}{l}\text { CF } \\
\text { GF }\end{array}$ & $\begin{array}{l}26.38 \\
27.13\end{array}$ & $\begin{array}{l}436.7 \\
381.2\end{array}$ & $\begin{array}{l}51.7 \\
19.6\end{array}$ & $\begin{array}{l}423.5 \\
205.1\end{array}$ & $\begin{array}{l}39.2 \\
16.2\end{array}$ & - & $\begin{array}{c}\text { Build } \\
\text { orientation, } \\
\text { layer }\end{array}$ & - \\
\hline & & $\mathrm{KF}$ & 27.13 & 305.2 & 25.5 & 189.8 & 14.1 & & $\begin{array}{l}\text { thickness, } \\
\text { fiber volume } \\
\text { content }\end{array}$ & \\
\hline \multirow[t]{2}{*}{$\begin{array}{c}\text { Mohammadizadeh } \\
\text { et al. [80] }\end{array}$} & Nylon & $\begin{array}{l}\text { CF } \\
\text { GF }\end{array}$ & $\begin{array}{l}58 \\
28\end{array}$ & $\begin{array}{l}404.3 \\
372.1\end{array}$ & - & - & - & - & $\begin{array}{c}\text { Fiber type, } \\
\text { fiber } \\
\text { orientations, }\end{array}$ & - \\
\hline & & $\mathrm{KF}$ & 43 & 309.14 & - & - & - & & temperatures & \\
\hline Hedayati et al. [52] & PCL & PGA suture & 22 & 380 & 79.7 & 3.5 & - & - & $\begin{array}{l}\text { Lay-down } \\
\text { pattern }\end{array}$ & - \\
\hline Tian et al. [102] & PLA & $\mathrm{CF}$ & 27 & - & - & 335 & 30 & - & $\begin{array}{c}\text { Temperature, } \\
\text { layer } \\
\text { thickness, } \\
\text { hatch spacing, } \\
\text { feed rate of } \\
\text { the filament, } \\
\text { printing speed }\end{array}$ & - \\
\hline
\end{tabular}




\begin{tabular}{|c|c|c|c|c|c|c|c|c|c|c|}
\hline Dong et al. [41] & Nylon & KF & 90 & 333.143 & 27 & - & - & - & $\begin{array}{c}\text { Fiber } \\
\text { orientation, } \\
\text { volume } \\
\text { fraction, the } \\
\text { position of } \\
\text { fibers }\end{array}$ & - \\
\hline $\begin{array}{c}\text { Naranjo-Lozada et } \\
\text { al. [84] }\end{array}$ & Nylon & $\mathrm{CF}$ & 54 & 304.28 & - & - & - & - & $\begin{array}{l}\text { Infill density, } \\
\text { infill patterns, } \\
\text { fiber volume } \\
\text { fraction, the } \\
\text { printing } \\
\text { architecture }\end{array}$ & - \\
\hline \multirow[t]{7}{*}{ Akhoundi et al. [28] } & PLA & GF & 30.5 & 234 & 19.4 & - & - & - & Nozzle system & - \\
\hline & PLA+ & GF & 31.5 & 268 & 19.7 & - & - & & & \\
\hline & PLA-wood & GF & 33.6 & 270 & 19.9 & - & - & & & \\
\hline & TPU & GF & 34.8 & 227 & 18.2 & - & - & & & \\
\hline & HDglass & GF & 31.3 & 285 & 19 & - & - & & & \\
\hline & POM & GF & 37.5 & 267 & 21.5 & - & - & & & \\
\hline & $\mathrm{PA}+\mathrm{CF}$ & GF & 36.3 & 234 & 20.8 & - & - & & & \\
\hline Ming et al. [77] & Epoxy & GF & 43 & 272.51 & 8.01 & 299.36 & 8.35 & Impregnation & UV curing & $\begin{array}{c}\text { Heat } \\
\text { treatment }\end{array}$ \\
\hline Yu et al. [113] & Onyx & $\mathrm{CF}$ & 48.72 & - & - & 270.63 & - & - & Infill pattern & - \\
\hline Tian et al. [101] & PLA & $\mathrm{CF}$ & 8.9 & 256 & 20.6 & 263 & 13.3 & $\begin{array}{c}\text { Recycled } \\
\text { impregnated } \\
\text { filament }\end{array}$ & - & - \\
\hline Peng et al. [87] & PLA & $\mathrm{CF}$ & 19.1 & - & - & 261.7 & 16 & - & $\begin{array}{l}\text { Raster angle, } \\
\text { stacking } \\
\text { sequence, } \\
\text { loading } \\
\text { direction }\end{array}$ & - \\
\hline
\end{tabular}




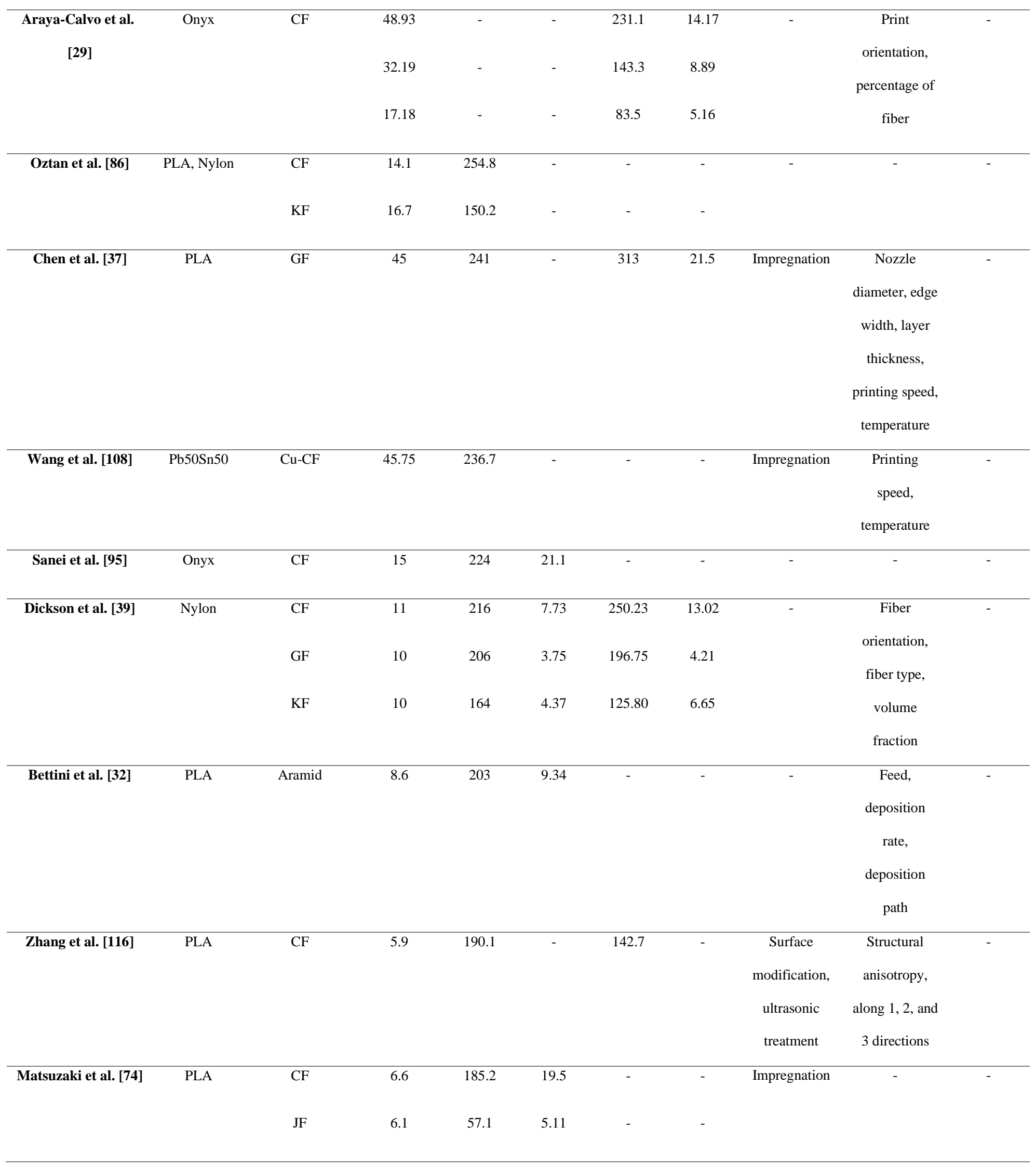




\begin{tabular}{|c|c|c|c|c|c|c|c|c|c|c|}
\hline Wang et al. [109] & PLA & GF & $\Delta, Y^{\prime}, 6.24$ & 171.66 & - & 148 & - & - & $\begin{array}{c}\text { Printing } \\
\text { temperature, } \\
\text { speed, layer } \\
\text { height, fiber } \\
\text { volume } \\
\text { fraction }\end{array}$ & - \\
\hline Vaneker et al. [106] & $\begin{array}{c}\text { polypropyle } \\
\text { ne (PP) }\end{array}$ & GF & - & - & - & - & 13.06 & - & $\begin{array}{l}\text { The cutting } \\
\text { device, } \\
\text { modified } \\
\text { deposition } \\
\text { strategy }\end{array}$ & - \\
\hline Qiao et al. [92] & PLA & $\mathrm{CF}$ & 5 & 164.8 & 3.2 & 174.4 & 9.2 & $\begin{array}{c}\text { Impregnation } \\
\text { with } \\
\text { ultrasonic } \\
\text { treatment }\end{array}$ & - & - \\
\hline Yang et al. [110] & ABS & $\mathrm{CF}$ & 10 & 147 & 4.185 & 127 & 7.72 & - & - & - \\
\hline Luo et al. [73] & PLA & $\mathrm{CF}$ & 40 & 143.11 & 4.05 & - & - & $\begin{array}{c}\text { Preparation } \\
\text { process }\end{array}$ & - & - \\
\hline Chen et al. [38] & Onyx & $\mathrm{CF}$ & 8.036 & 136 & 1.276 & - & - & - & $\begin{array}{l}\text { Average and } \\
\text { central mode } \\
\text { structures }\end{array}$ & - \\
\hline Prajapati et al. [88] & Onyx & $\begin{array}{c}\text { GF } \\
\text { HSHT GF }\end{array}$ & $\begin{array}{l}30.1 \\
30.1\end{array}$ & $\begin{array}{l}126.1 \\
131\end{array}$ & - & $\begin{array}{l}- \\
-\end{array}$ & $\begin{array}{l}- \\
-\end{array}$ & - & - & - \\
\hline Ipekci et al. [60] & $\begin{array}{c}\text { photopolym } \\
\text { er }\end{array}$ & GF & - & 125 & - & 450 & 14.5 & - & $\begin{array}{c}\text { Nozzle } \\
\text { diameter, } \\
\text { printing speed, } \\
\text { fiber density, } \\
\text { and ultraviolet } \\
\text { (UV) light } \\
\text { intensity }\end{array}$ & - \\
\hline Mosleh et al. [82] & ABS & $\mathrm{CF}$ & 11.4 & 119.85 & - & 102.6 & - & Impregnation & $\begin{array}{c}\text { Nozzle } \\
\text { diameter, }\end{array}$ & - \\
\hline
\end{tabular}




\begin{tabular}{|c|c|c|c|c|c|c|c|c|c|c|}
\hline & & & & & & & & & $\begin{array}{l}\text { layer height, } \\
\text { print speed }\end{array}$ & \\
\hline Chaudhry et al. [36] & PLA & $\mathrm{CF}$ & 1.7 & 112 & 0.97 & 164 & 8.528 & - & $\begin{array}{c}\text { Number of } \\
\text { layers, } \\
\text { material } \\
\text { impact, } \\
\text { interlayer gap }\end{array}$ & - \\
\hline Yin et al. [112] & PLA & $\mathrm{CF}$ & 9.62 & 111 & 12.2 & 152.9 & 9.5 & - & $\begin{array}{l}\text { Number of } \\
\text { layers, hatch } \\
\text { space, filling } \\
\text { angle }\end{array}$ & - \\
\hline Mei et al. [75] & Nylon & $\begin{array}{l}\mathrm{CF} \\
\mathrm{GF} \\
\mathrm{KF}\end{array}$ & - & $\begin{array}{l}110 \\
91 \\
75\end{array}$ & $\begin{array}{l}3.941 \\
1.826 \\
2.044\end{array}$ & - & - & - & $\begin{array}{l}\text { Fiber layers, } \\
\text { infill pattern }\end{array}$ & - \\
\hline $\begin{array}{c}\text { Gonzalez-Estrada et } \\
\text { al. [50] }\end{array}$ & Nylon & $\begin{array}{l}\mathrm{CF} \\
\mathrm{GF}\end{array}$ & - & $\begin{array}{l}104 \\
83\end{array}$ & $\begin{array}{l}4.431 \\
1.61\end{array}$ & $\begin{array}{l}- \\
-\end{array}$ & $\begin{array}{l}- \\
-\end{array}$ & - & $\begin{array}{l}\text { Fiber } \\
\text { orientation, } \\
\text { infill density } \\
\text { \& pattern, } \\
\text { reinforcement } \\
\text { distribution }\end{array}$ & - \\
\hline Prajapati et al. [90] & Onyx & HSHT GF & 30 & 94.2 & - & - & - & - & - & - \\
\hline Li et al. [68] & PLA & $\mathrm{CF}$ & 34 & 91 & - & 156 & - & $\begin{array}{c}\text { Surface } \\
\text { preprocessing } \\
\text { modification }\end{array}$ & $\begin{array}{c}\text { Extrusion } \\
\text { nozzle, } \\
\text { printing path } \\
\text { control }\end{array}$ & - \\
\hline Shi et al. [97] & Nylon & $\mathrm{KF}$ & 8.43 & 88.18 & - & - & - & - & $\begin{array}{c}\text { Layer } \\
\text { distributions, } \\
\text { fiber } \\
\text { orientations }\end{array}$ & - \\
\hline Dong et al. [42] & PLA & $\mathrm{KF}$ & 16.32 & 87.36 & & - & - & - & $\begin{array}{l}\text { Cell length, } \\
\text { layer } \\
\text { thickness }\end{array}$ & - \\
\hline
\end{tabular}




\begin{tabular}{|c|c|c|c|c|c|c|c|c|c|c|}
\hline Liu et al. [69] & $\begin{array}{c}\text { PA6, } \\
\text { Carbon } \\
\text { nanotube } \\
\text { (CNT), } \\
\text { graphene } \\
\text { nano } \\
\text { platelet } \\
\text { (GNP) }\end{array}$ & KF & $1 \%$ GNP & 80.4 & - & - & - & - & Nanofillers & - \\
\hline $\begin{array}{c}\text { Heidari-Rarani et } \\
\text { al. [53] }\end{array}$ & PLA & $\mathrm{CF}$ & 28.2 & 61.4 & 8.28 & 152.1 & 13.42 & $\begin{array}{c}\text { Fiber surface } \\
\text { preparation }\end{array}$ & $\begin{array}{l}\text { Innovative } \\
\text { extruder, } \\
\text { printing } \\
\text { temperature, } \\
\text { feed rate }\end{array}$ & - \\
\hline Baumann et al. [31] & ABS & $\begin{array}{l}\mathrm{CF} \\
\mathrm{GF}\end{array}$ & $\begin{array}{l}0.3 \\
0.6\end{array}$ & $\begin{array}{l}41 \\
49\end{array}$ & $\begin{array}{c}r \\
r / .0\end{array}$ & - & $\begin{array}{l}- \\
-\end{array}$ & - & $\begin{array}{c}\text { Implementing } \\
\text { fibers }\end{array}$ & - \\
\hline Mori et al. [81] & ABS & $\mathrm{CF}$ & 1.6 & 43 & - & - & - & - & & \\
\hline Cersoli et al. [34] & PLA & $\mathrm{KF}$ & 20.53 & 84.1 & 3.68 & 84.9 & 2.68 & - & $\begin{array}{l}\text { Fiber volume } \\
\text { fraction }\end{array}$ & - \\
\hline Yao et al. [111] & PLA & $\mathrm{CF}$ & - & 32.570 & - & 68.211 & - & $\begin{array}{c}\text { CFs } \\
\text { impregnated } \\
\text { with DY-E44 } \\
\text { resin }\end{array}$ & - & - \\
\hline Shang et al. [26] & PLA & $\mathrm{CF}$ & 15 & 32.1 & 3.347 & - & - & - & $\begin{array}{c}\text { Amplitude \& } \\
\text { frequency of } \\
\text { structural } \\
\text { parameters }\end{array}$ & - \\
\hline
\end{tabular}




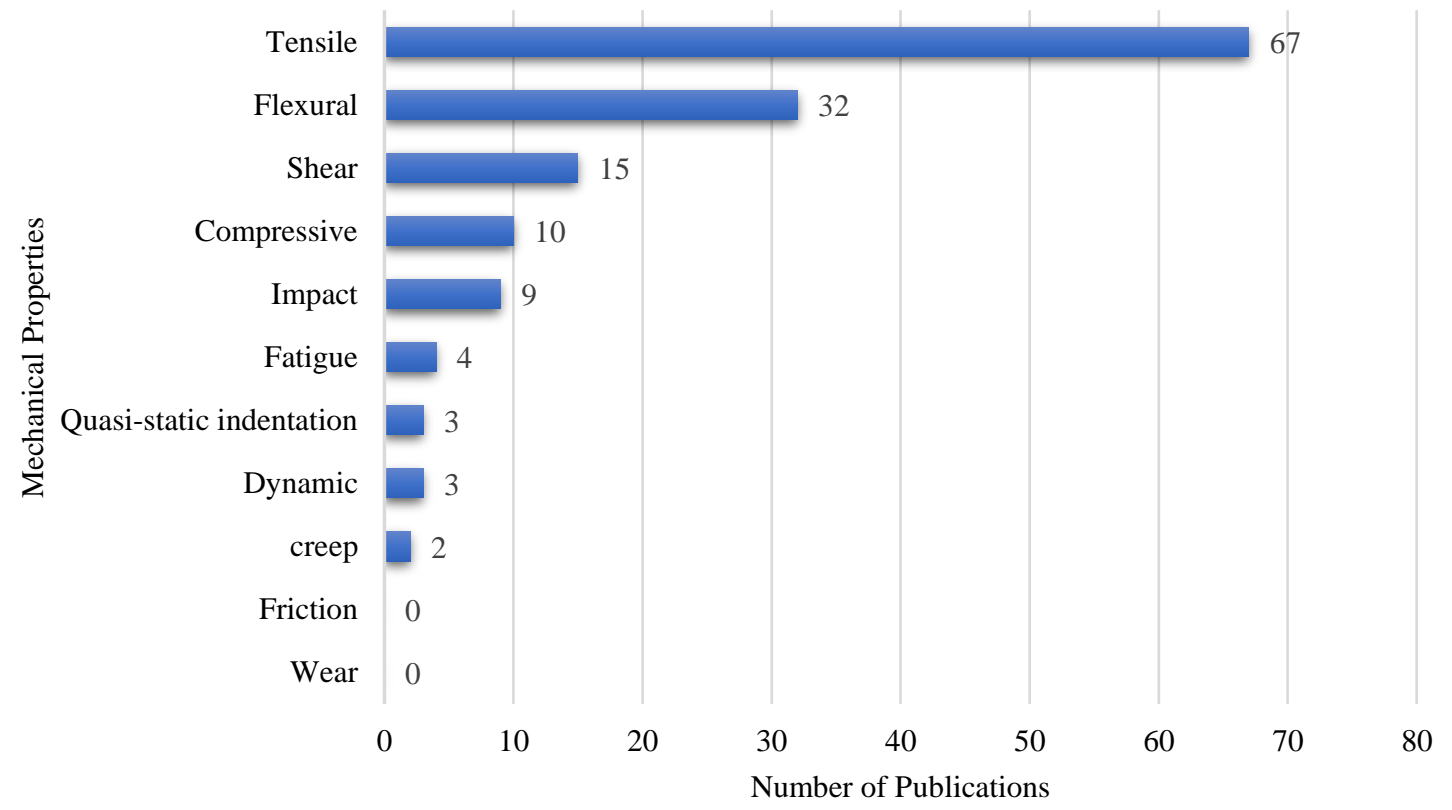

Fig. 6 Various mechanical characteristics, and the number of publications that have examined them

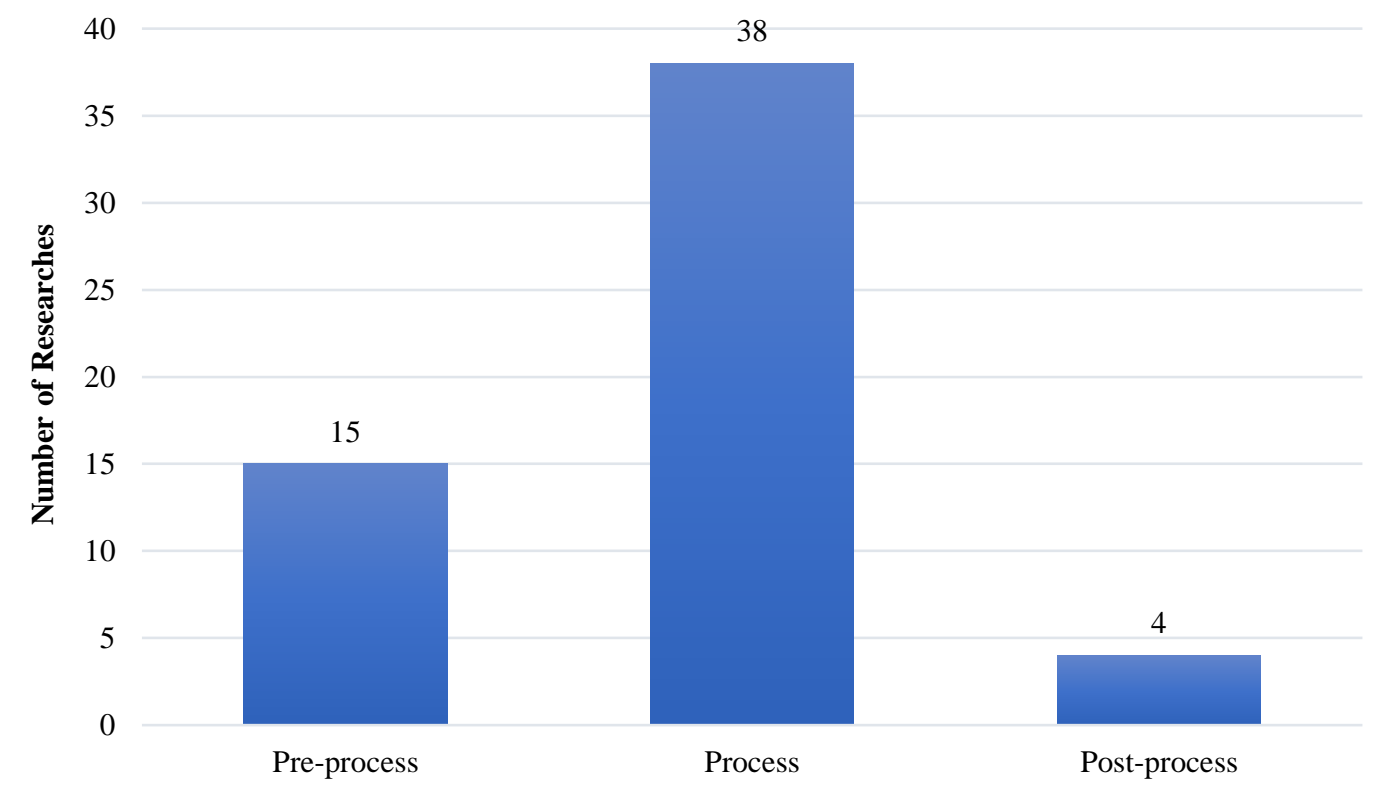

Fig. 7 Number of researches done on Pre-process, process, and postprocessing of CFRCs 


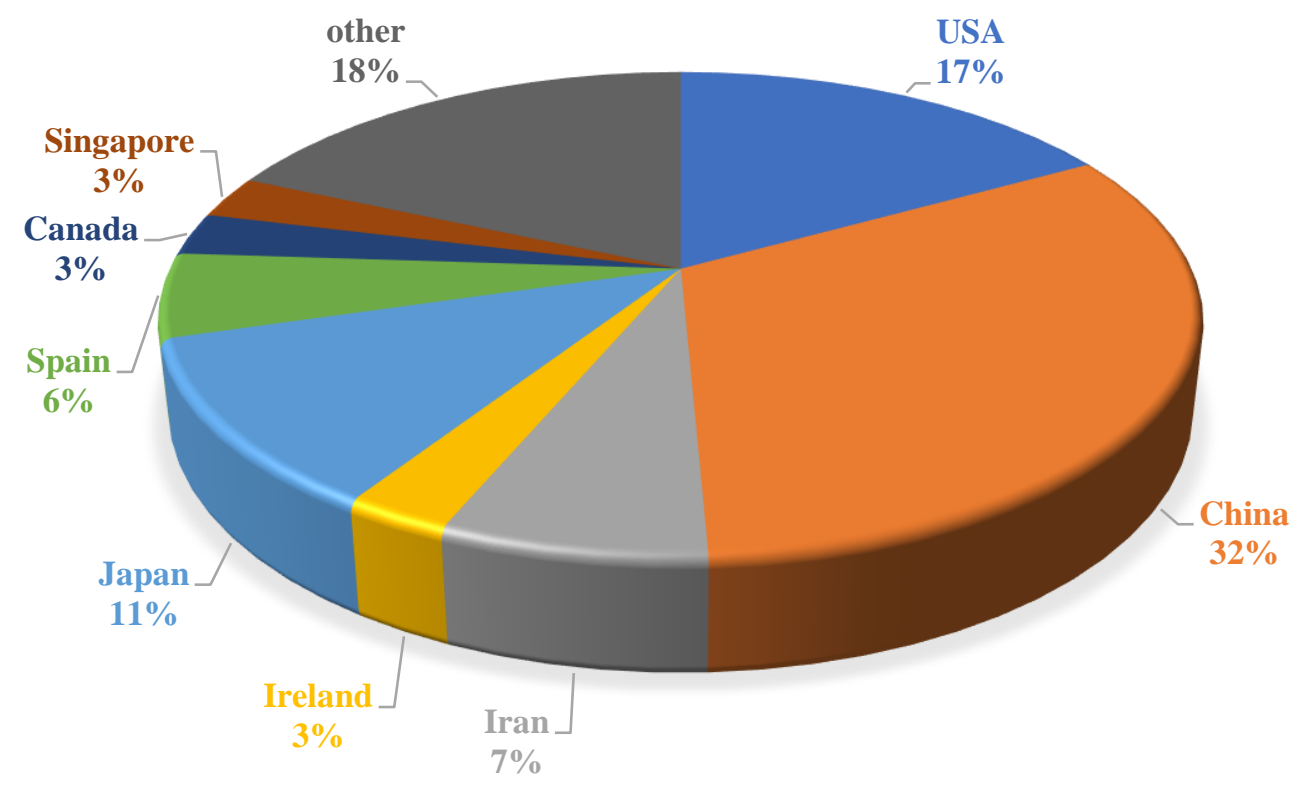

Fig. 8 Published articles in the field of CFRC 3D printing by country

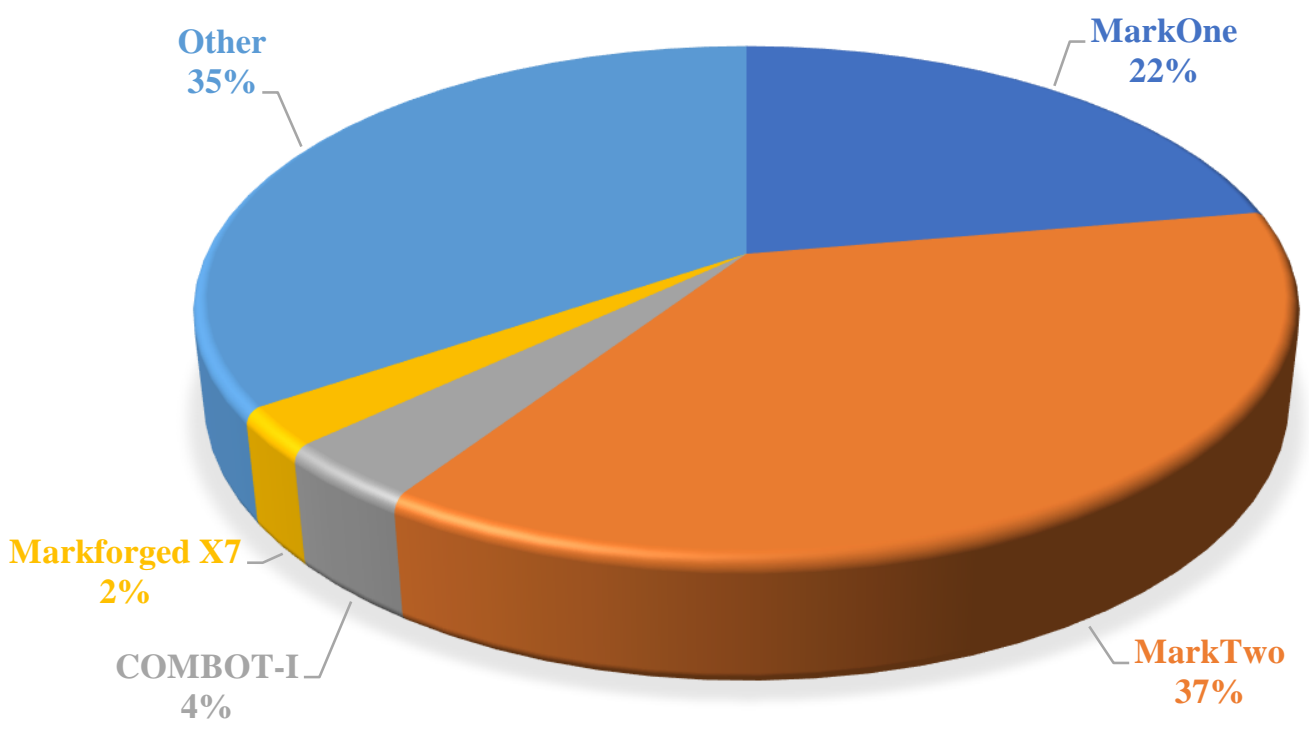

Fig. 9 Printers used for 3D printing of CFRCs 


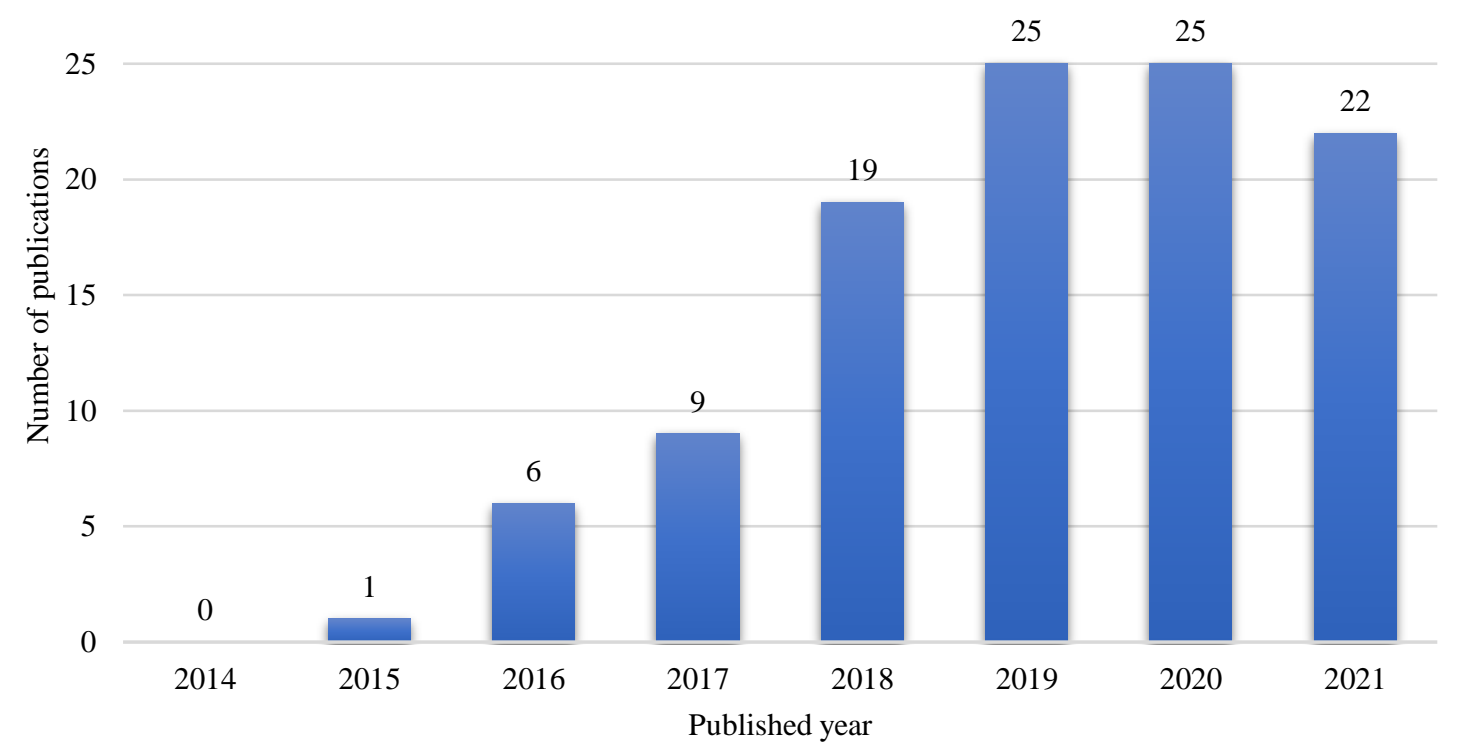

Fig. 10 Number of CFRC 3D printing articles published each year

\section{Pre-processing conditions}

\subsection{Impregnation}

As

indicated

in

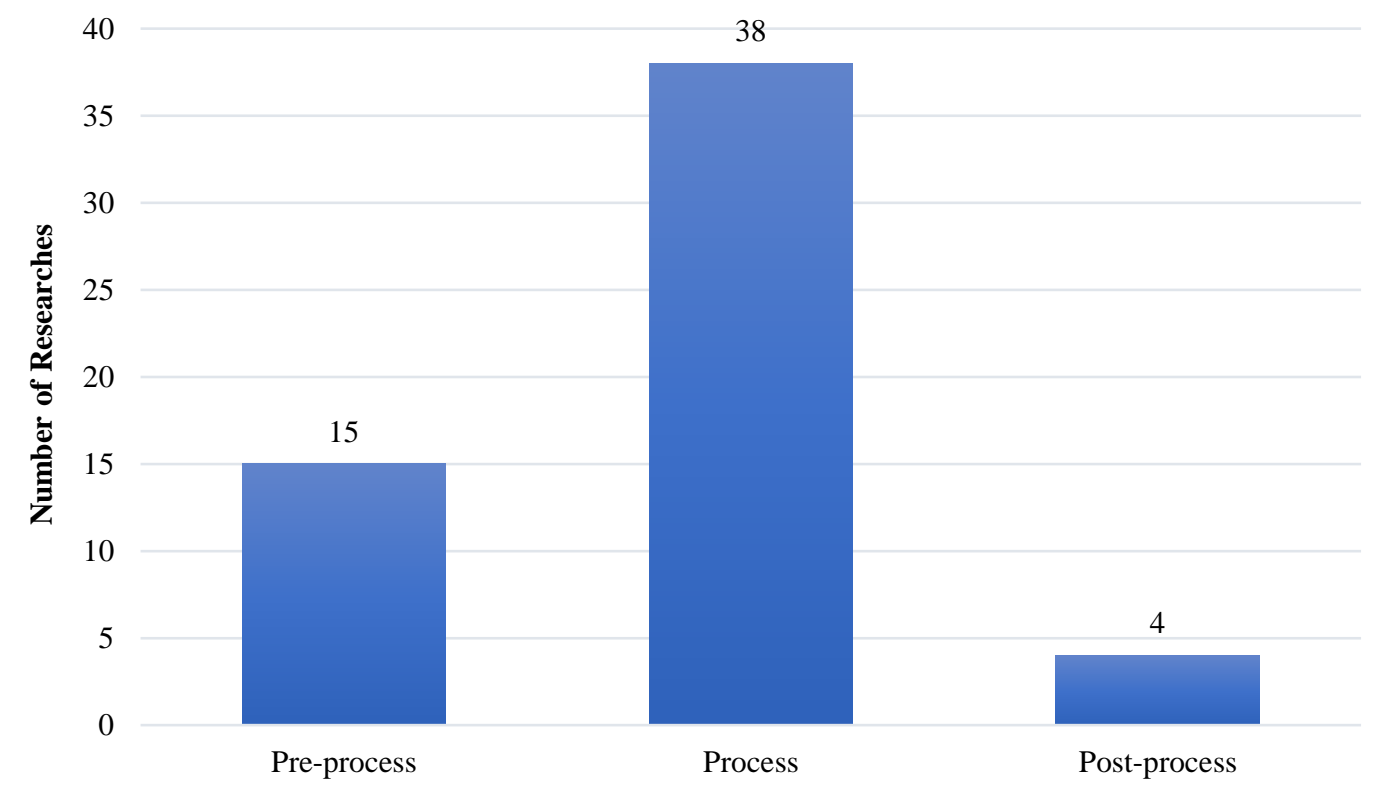


Fig. 7, pre-processing was the subject of 15 of the papers evaluated (mostly on impregnation). The application of impregnation is shown schematically in Fig. 11. The findings revealed that impregnation quality has a significant impact on mechanical characteristics. 
(a)

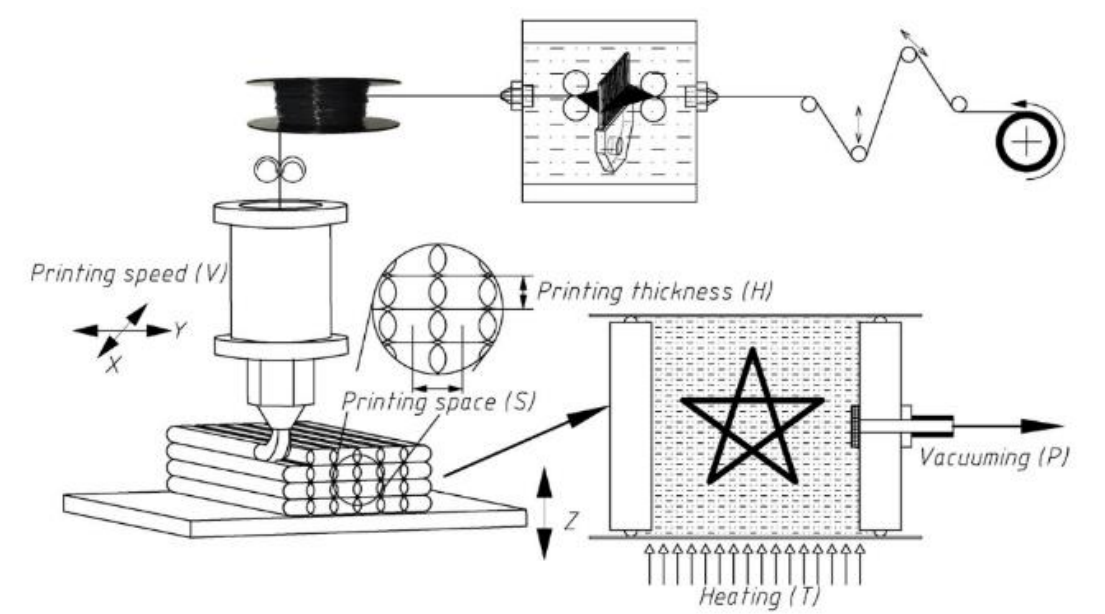

(c)

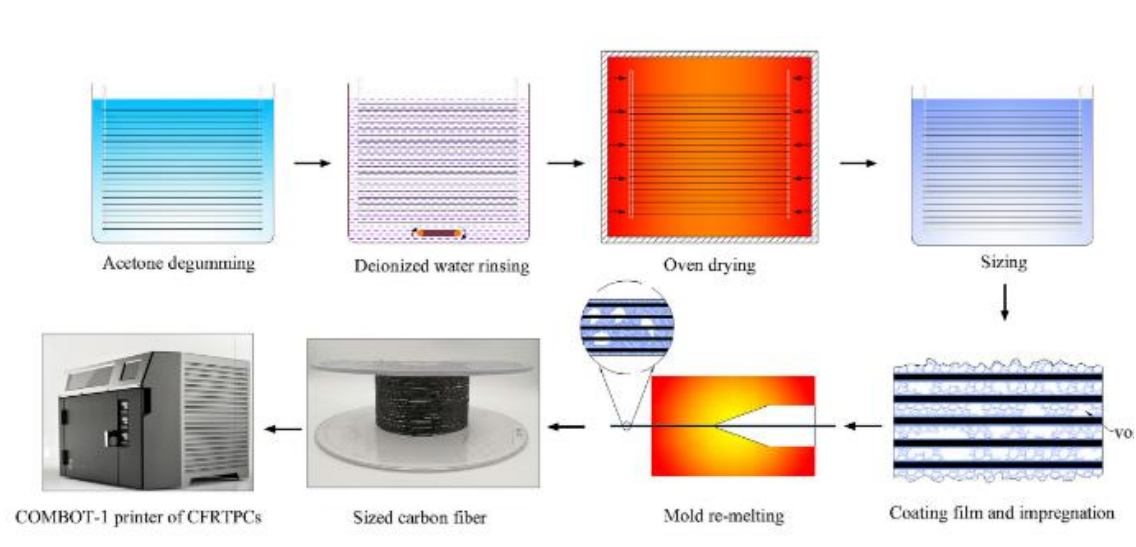

(b)

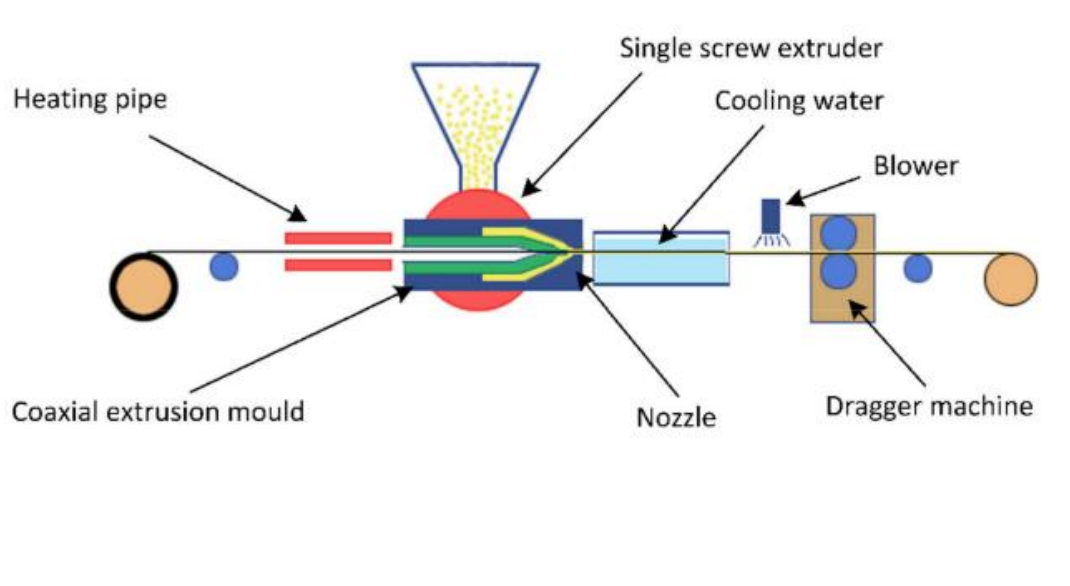

(d)

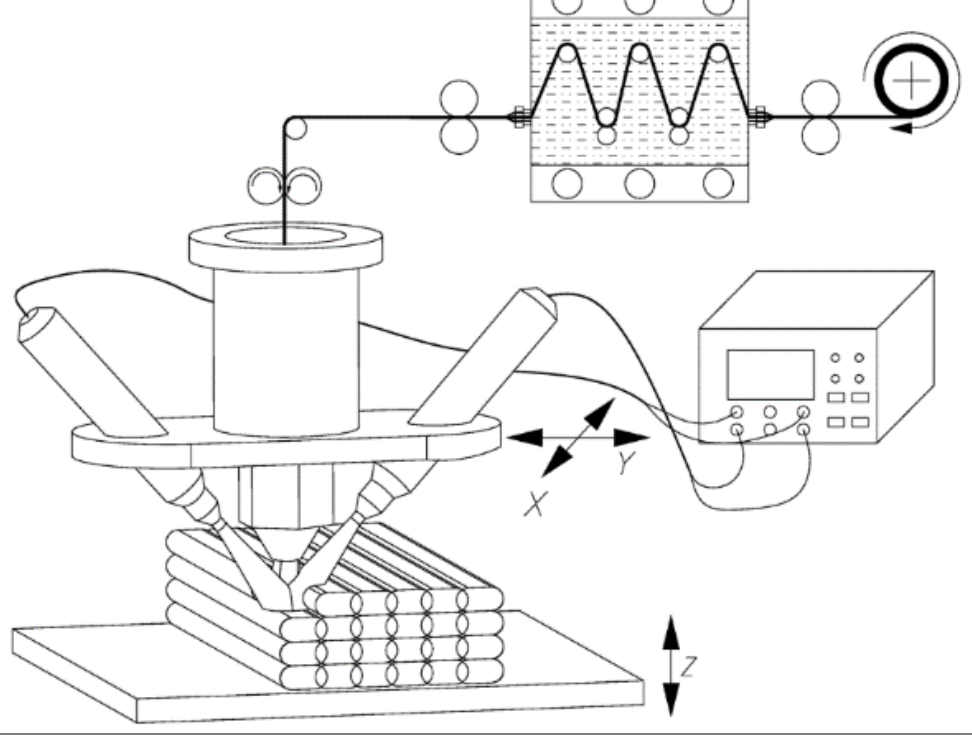




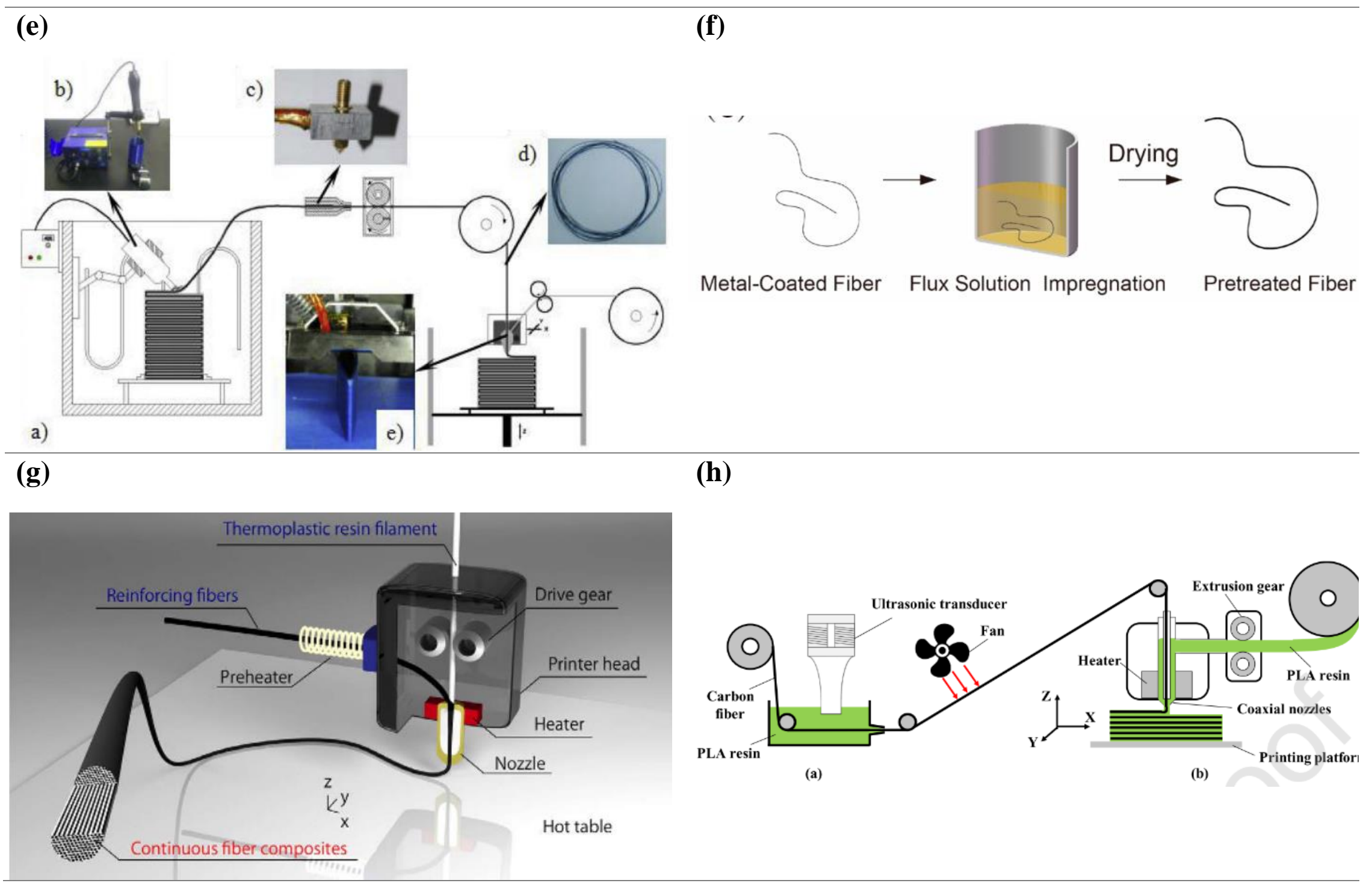




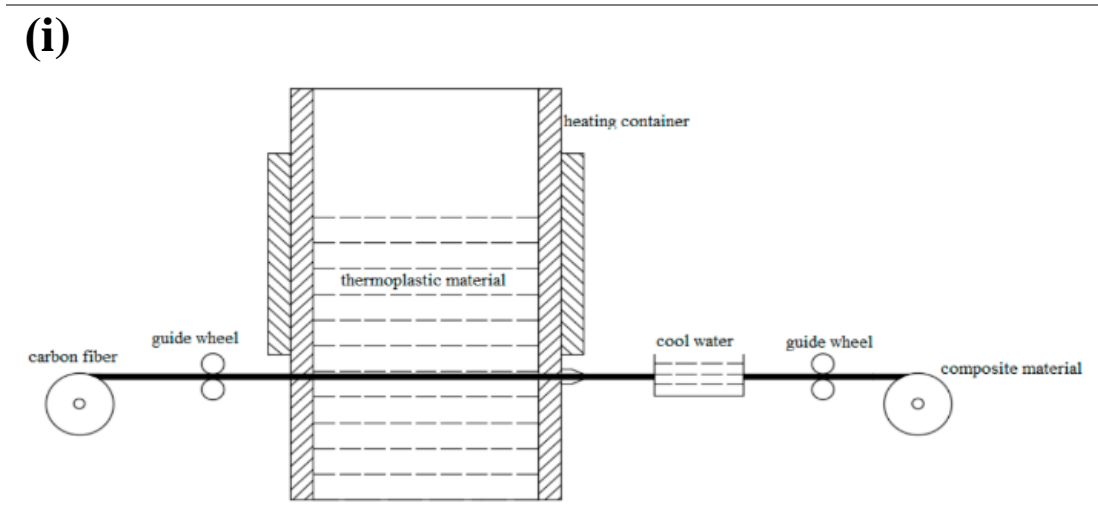

(k)

Methylene dichloride
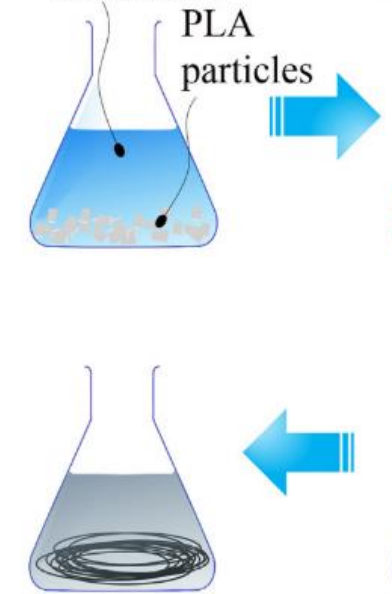

Infiltration of carbon fibers

Filtration
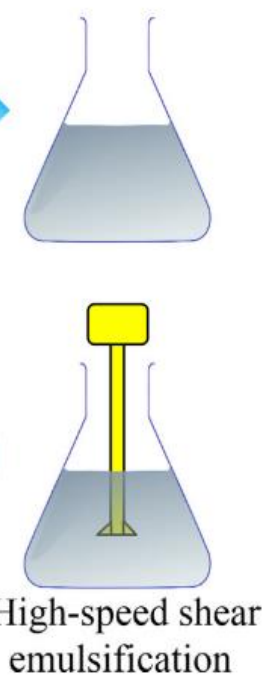

(j)

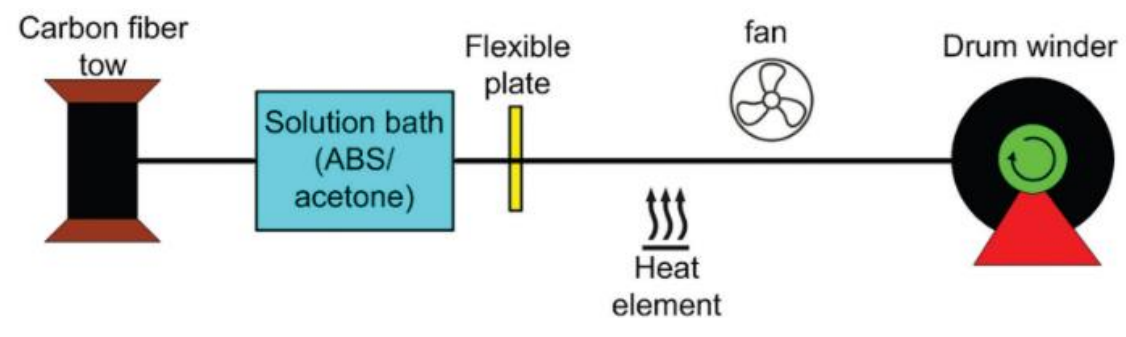

(I)

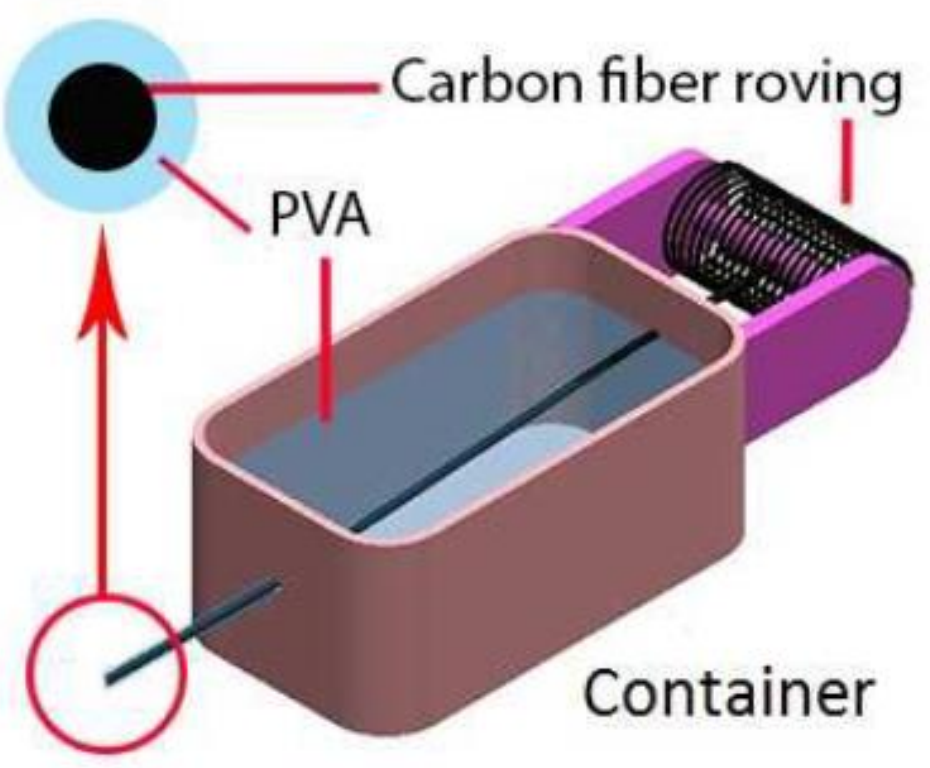




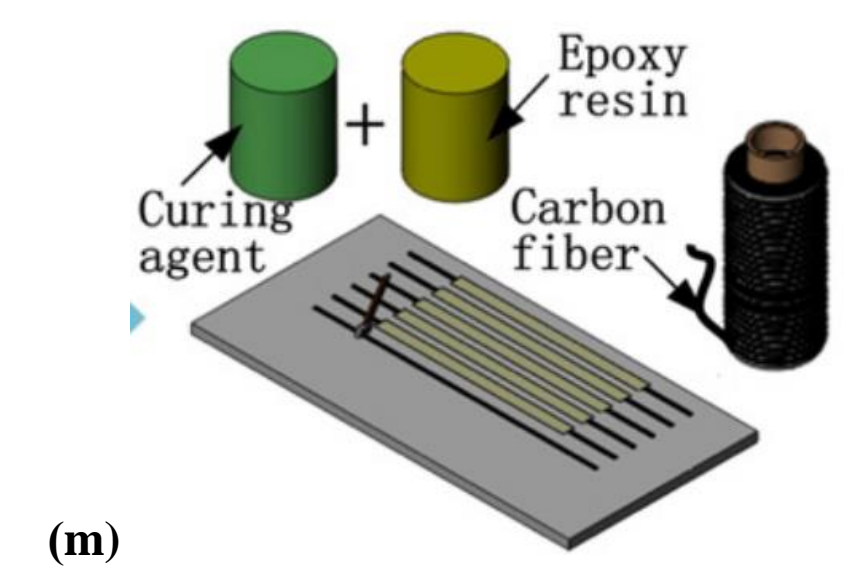

Fig. 11 Different simultaneous impregnation systems. (a) Ming et al. [78], (b) Hu et al. [56], (c) Liu et al. [71], (d) Ming et al. [77], (e) Tian et al. [101], (f) Wang et al. [108], (g) Matsuzaki et al. [74], (h) Qiao et al. [92], (i) Luo et al. [73], (j) Mosleh et al. [82], (k) Li et al. [68], (1) Heidari-Rarani et al. [53], and (m) Yao et al. [111] 
By impregnating $3 \mathrm{~K}$ CFs with the thermosetting matrix at $130^{\circ} \mathrm{C}$, Ming et al. [78] created the required 3D printing filament. Because various deflections along the impregnation path and adjustment of the conveying tension may enhance the impregnation area, the method used many yarn rollers and spreading needles to stretch the fibers for a greater impregnation result (see Fig. 11(a)). To make the continuous CF prepreg filament, Hu et al. [56] used a single screw extruder and coaxial extrusion molds. The liquid resin has a higher pressure because of the single screw extruder, which may compress the molten resin into the continuous $\mathrm{CF}$ filaments in the nozzle. Apart from guaranteeing that the resin is readily squeezed into the filaments, the coaxial extrusion mold, particularly its nozzle, also generates a resin barrier to protect the inner continuous $\mathrm{CF}$ filament. The continuous $\mathrm{CF}$ was heated in the heating pipe before entering the coaxial extrusion mold to keep it dry and warm and guarantee that the filaments are readily saturated with the molten resin. After impregnation, the continuous CF prepreg filament was placed in cooling water to solidify, and a fan was employed to maintain the filament dry in the next phase. The dragger machine provided the necessary power to keep the entire production process running smoothly (see Fig. 11(b)).

According to Liu et al. [71], the virgin CF was first extracted with acetone for 48 hours, then repeatedly rinsed with deionized water and dried in an oven at $100^{\circ} \mathrm{C}$ for 2 hours to remove the original epoxy sizing layer. The CF was then immersed in the aqueous PA845H solution for 12 hours to guarantee that the polyamide solids penetrated the fiber bundle completely. Following that, the $\mathrm{CF}$ was aired at room temperature while polyamide particles were deposited on each fiber surface to produce a sizing film, and some polyamide solids were also present between fibers to form pre-impregnation. The distribution of polyamide solids, on the other hand, was uneven, with some gaps in the fiber bundle and a rough visible surface. As a result, a remolding nozzle with a 
diameter of $0.4 \mathrm{~mm}$ suggested a melt impregnation procedure. The pre-impregnated $\mathrm{CF}$ was continually pushed through the nozzle at a speed of $10 \mathrm{~mm} / \mathrm{s}$, while the polyamide solids were melted at $200{ }^{\circ} \mathrm{C}$ to mix and fill the gaps in the nozzle under pressure to achieve uniform distribution and a smooth surface. Finally, the CFRCs method employed sized CF and PA6 filament as raw materials to build composite components using a 3D printer (see Fig. 11(c)). The procedure was easy and cheap, and it enhanced the interlaminar shear strength of the PA6/sized CF sample by $42.2 \%$.

Ming et al. [77] used a thermosetting matrix with a low viscosity of 1.3 Pa.s (Pascal-second) at $130^{\circ} \mathrm{C}$ to impregnate continuous glass fibers (GFs). In the molten resin tank, many yarn rollers were used to stretch the fiber bundles and guarantee a broad resin impregnation area. The stretched fiber bundles were then molded into a cylinder form using a squeezing nozzle, the excess resin scraped off, and the impregnated continuous GFs extruded through a squeezing nozzle (see Fig. 11(d)). Tian et al. [101] presented a recycling and remanufacturing method for 3D printed CFRC. From 3D printed composite components, continuous CF and PLA matrix were recycled in the form of PLA impregnated CF filament. With the action of the hot air cannon, they softly and consistently took out the CF. The impregnated CF filament with a somewhat rough surface was produced by resolidified thermoplastic material adhering to the fiber (see Fig. 11(e)). Due to enhanced surface characteristics, impregnated recycled continuous $\mathrm{CF}$ has a greater tensile strength $(142 \mathrm{~N})$ than the original printed filament $(118 \mathrm{~N})$.

Wang et al. [108] submerged the $\mathrm{Cu} / \mathrm{CF}$ in flux solution for 1 minute before drying it at ambient temperature $\left(25^{\circ} \mathrm{C}\right)$. Matsuzaki et al. [74] heated the reinforcing fibers with a nichrome wire before introducing them into the nozzle to improve the permeation of the fiber bundles with thermoplastic resin; the heat diffuses to the resin, lowering the PLA viscosity. Drive gears and a stepping motor 
transport the resin filament, while reinforcing fibers are sent directly to the nozzle. The reinforcing fibers are automatically fed to the head by the movement of the resin filament, thus no extra equipment is necessary for feeding them. The heater within the printer head melts the resin filament, merging the reinforcing fibers and resin in the heated area.

By dissolving the thermoplastic resin in dichloromethane, Qiao et al. [92] created a resin solution. The CF bundle is then dragged into the resin solution pool by the winding machine. The CF bundle maintains a constant tension throughout the movement thanks to the rotating dampers (as shown in Fig. 11(a)). The CF bundle is $10 \mathrm{~mm}$ away from the ultrasonic transducer and entirely immersed in the resin solution. Following that, under the cavitation of the ultrasonic transducer, the resin solution penetrates deeper into the CF bundle. The impregnated CF bundle is then passed through a scraping hole to scrape off any extra resin on its surface, and the dichloromethane solvent is volatilized under hot air. Finally, as a printing raw material, the pretreated CF bundle is wound on the winding machine reel. The resin solution pool is kept sealed throughout the operation to prevent solvent evaporation, the ultrasonic transducer is kept cold with flowing water to prevent resin sticking, and the CF solvent evaporation process is carried out in the fume hood (see Fig. 11(h)). The influence of ultrasonic pretreatment parameters such as amplitude, resin solution concentration, and treatment speed on the mechanical characteristics of printed components has been investigated. The results show that when the amplitude increases, the tensile strength, and elastic modulus rise first, then fall. The tensile and flexural strength and modulus reach their maximum when the amplitude is $40 \mu \mathrm{m}$, resulting in tensile strength and modulus of $164.8 \mathrm{MPa}$ and 3.2 GPa, respectively. The tensile strength and the tensile and flexural modulus of the material rose as the percentage of resin solution concentration increased. Strength, tensile, and flexural modulus decrease as treatment speed rises. 
The dry CF tow was run through the whole preparation apparatus by Luo et al. [73]. The PLA particles are then heated to a molten state at $210^{\circ} \mathrm{C}$ in a sealed container. At the same temperature, the two materials are combined. They will then travel at a speed of $10 \mathrm{~mm} / \mathrm{s}$ through a $0.6 \mathrm{~mm}$ nozzle. Finally, they are chilled in room temperature water $\left(25^{\circ} \mathrm{C}\right)$ to produce a CCF PLA composite material (see Fig. 11(i)). Mosleh et al. [82] developed solution impregnated fibers (prepregs). In a closed vessel at room temperature, $8 \mathrm{~g}$ ABS was swirled in $100 \mathrm{~mL}$ acetone for 30 minutes. To avoid compatibility concerns, the impregnation solution was made with the same ABS grade printing filament. A drum winder was used to draw the $\mathrm{CF}$ through the solution bath. To remove the surplus solution from prepregs, a flexible plate with a certain hole size was employed (Fig. 11(j)). The findings demonstrate that impregnation before printing enhances mechanical characteristics and simplifies 3D printing.

PLA particles (8 percent mass fraction) were introduced to a methylene dichloride solution by $\mathrm{Li}$ et al. [68]. After 30 minutes of magnetic stirring, the PLA particles were partly dissolved. The DE100LB high-speed dispersion and emulsification machine shears and emulsifies the PLA resin filtrate in methylene dichloride solution at 8000 revolutions per minute. With a 1 percent mass fraction of the whole solution, the surface-active, emulsifying, and antifoaming agents are added to the deionized water. The deionized water is then progressively added to process the aqueous PLA sizing agent to change the surface condition of the CFs (see Fig. 11(k)). The tensile and flexural strengths of modified CFRCs were $13.8 \%$ and $164 \%$ greater than the original CF reinforced samples, according to the data. The modified CF reinforced samples had a storage modulus that was $166 \%$ and $351 \%$ greater than the PLA and original fiber reinforced samples, respectively. 
Heidari-Rarani et al. [53] dissolved roughly 400g water-soluble polyvinyl alcohol (PVA) in 2 L water in a container and impregnated $\mathrm{CF}$ roving in this solution for 1 hour at $60^{\circ} \mathrm{C}$. The impregnated roving is passed through a die with a $1 \mathrm{~mm}$ hole and dried at room temperature to remove the excess PVA and create an approximate circular cross-section (see Fig. 11(1)). For the CF preparation, Yao et al. [111] impregnated a $250 \mathrm{~mm}$ length of fiber with a two-component (1:1) DY-E44 epoxy resin adhesive.

\section{Process (printing) conditions}

Similar to 3D printing of neat thermoplastics, the quality of 3D printed CFRCs is influenced by a variety of variables. The bulk of CFRC 3D printing research has focused on printing (processing) parameters rather than pre- and post-processing conditions (see

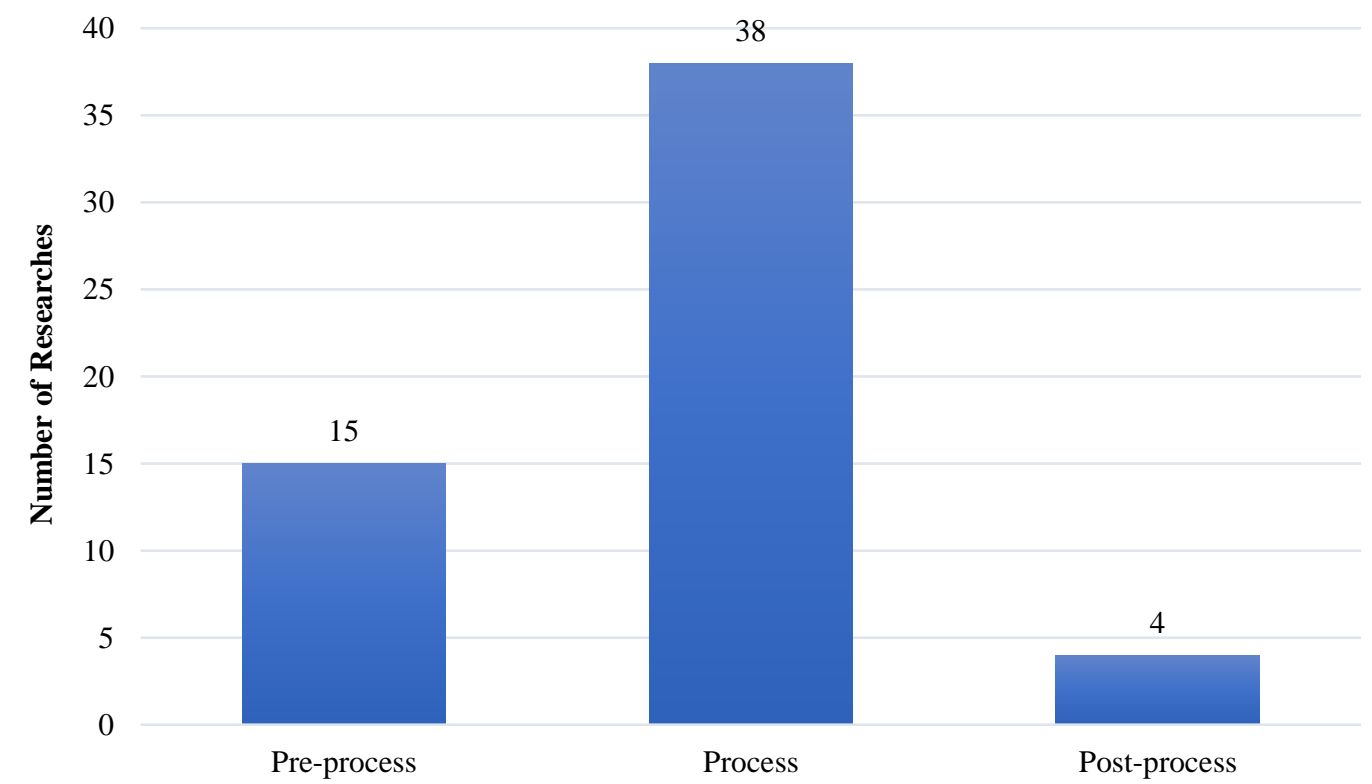

Fig. 7). Process factors can have a significant influence on mechanical properties, according to the literature. Table 3 outlines the procedures utilized in the literature, with a focus on tensile and flexural testing. Fig. 12 depicts the investigated process variables. Each of these variables will be discussed in more detail. 


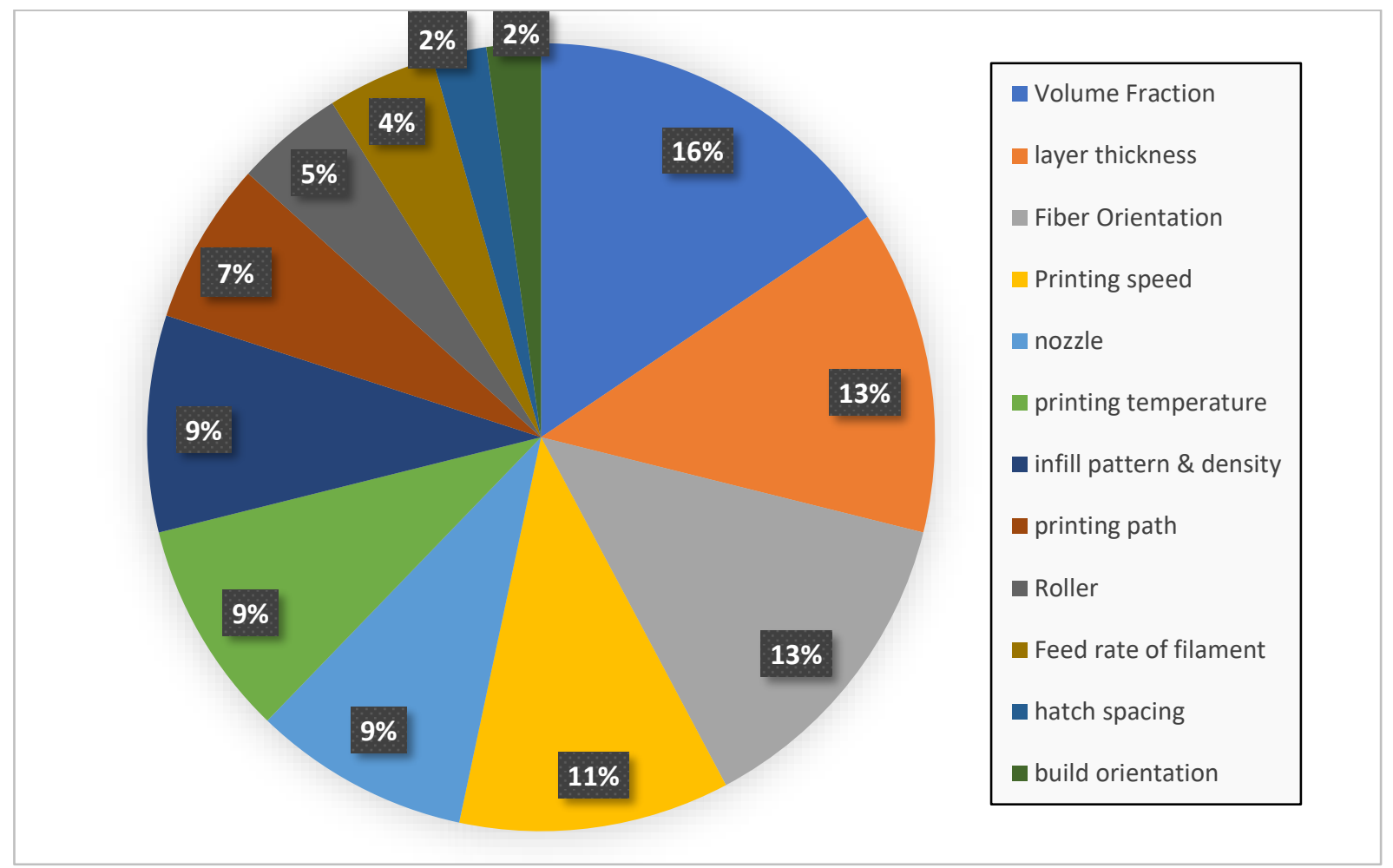

Fig. 12 Processing (printing) parameters assessed in the literature

\subsection{Fiber Volume Fraction}

The fiber volume fraction (FVF) of CFRCs, as well as their tensile and flexural strength and modulus, are listed in Table 3. To further understand the relationship between the fraction of FVF and the mechanical properties of CFRCs, the data in Table 3 is presented in Fig. 13 and Fig. 14. 


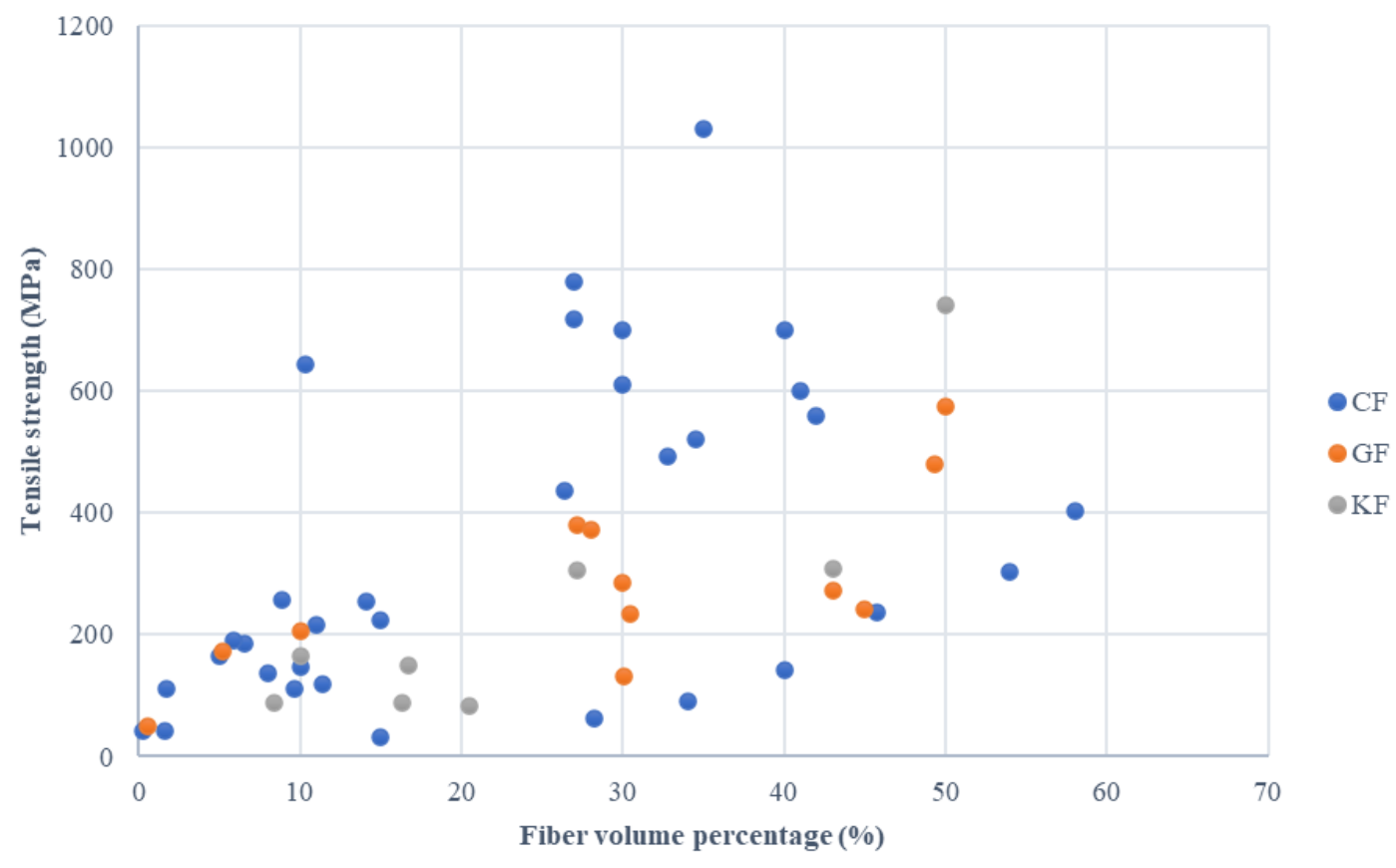

Fig. 13 Relation between FVF and Tensile Strength

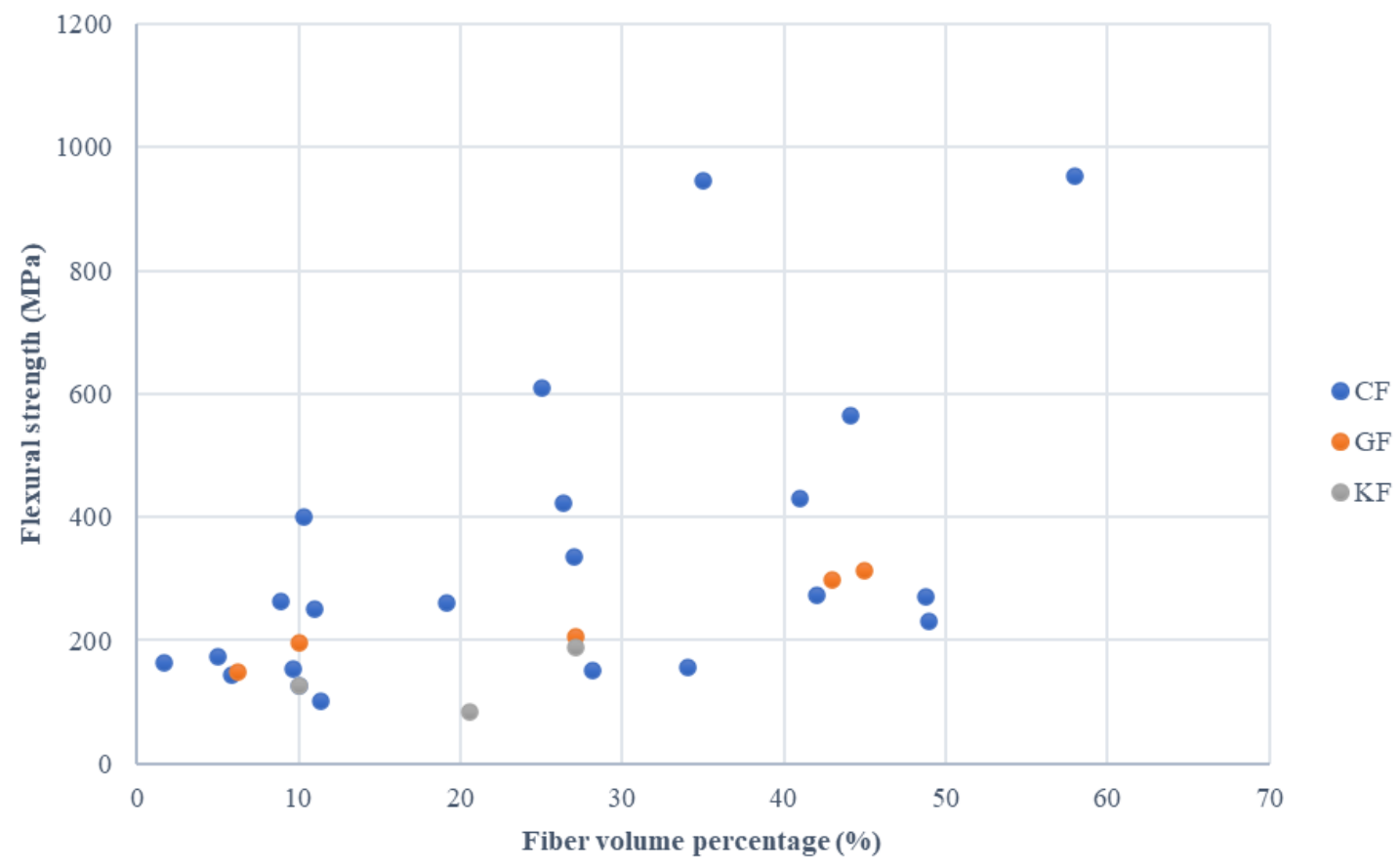

Fig. 14 Relation between FVF and Flexural Strength 
Hou et al. [55] investigated the mechanical characteristics of PLA/Kevlar composites with different fiber volume contents $(6.7,10,12.5,20,30,40,50$ percent). The findings reveal that increasing the fiber volume content of the samples from 6.7 to $50 \%$ improves longitudinal tensile strength from 141.8 $\mathrm{MPa}$ to $742.6 \mathrm{MPa}$, but decreases transverse tensile strength, longitudinal compressive strength, transverse compressive strength, and in-plane shear strength. Furthermore, if the fiber volume content of the samples is increased by $40 \%$, the longitudinal tensile and compressive moduli will increase to $41.3 \mathrm{GPa}$ and $5.89 \mathrm{GPa}$, respectively. These moduli do not rise by a further increase of the fiber volume content increases from $40 \%$ to $50 \%$.

R3, R4, R5, and R6 were the four samples examined by Akhoundi et al. [17], with fiber volumes of $49.3,46.3,40.18$, and 35.14 percent, respectively. The results show that as the percentage of fiber volume increases, the tensile strength and tensile modulus increase, with tensile strengths of $479 \mathrm{MPa}, 446 \mathrm{MPa}, 401 \mathrm{MPa}$, and $333 \mathrm{MPa}$ for samples R3, R4, R5, and R6, and tensile modulus of $29.41 \mathrm{GPa}, 27.76 \mathrm{GPa}, 24.31 \mathrm{GPa}$, and 21.9 GPa for samples R3, R4, R5, and R6, respectively.

Chacon et al. [35] used continuous carbon, glass, and Kevlar fibers as reinforcing material in printed nylon matrix composites. The fiber volume content of the samples varied from 1.88 to 27.13 percent for each of the reinforcing fibers. The results show that as the fiber volume content of the samples increases, so does their tensile and flexural strength. For example, when the fiber volume content of the samples changes from 1.88 to 27.13 , the tensile strength improves from 96.6 $\mathrm{MPa}$ to $436.7 \mathrm{MPa}$, and the strength bending improves from $80.7 \mathrm{MPa}$ to $423.5 \mathrm{MPa}$, and the tensile modulus increases from 7.6 GPa to $51.7 \mathrm{GPa}$, and the flexural modulus increases from 6.1 GPa to $39.2 \mathrm{GPa}$. 
The impact of fiber volume content in nylon/CF composites was studied by Naranjo-Lozada et al. [84]. The samples' fiber volume fractions are 4, 7, 11,32, and 54 percent, respectively. The results reveal that samples with a greater fiber volume percentage had higher strength and tensile modulus.

For PA6/CF composites, Araya-Calvo et al. [29] conducted bending tests on samples with 17.18, 32.19, and 48.93 percent fiber volume fraction, as well as compression testing on samples with $8.18,16.59$, and 24.44 percent. The modulus and proportional limit of bending and compression have grown as the amount of reinforcement have increased. The proportional limit of bending for the samples is $83.5 \mathrm{MPa}, 143.3 \mathrm{MPa}$, and $231.1 \mathrm{MPa}$, respectively. The samples' bending moduli are $5.16 \mathrm{GPa}, 8.89 \mathrm{GPa}$, and $14.17 \mathrm{GPa}$, respectively.

Dickson et al. [39] investigated the impact of FVF on the mechanical performance of a nylon matrix composite reinforced with carbon, glass, and Kevlar fibers. Volume fractions of 4, 9, 13.5, $18,22.5,27$, and 33 percent were used to make the composites. The results show that when the FVF of the samples is increased from 4 to 13.5, the strength of the sample is increased by 516 percent. but when the volume fraction is increased to 33 , the strength of the sample volume fraction increases by 13.5 percent, or 516 percent. By increase of the volume fraction of samples from 13.5 to 33 percent, 44 percent increase in strength occurred, resulting in maximum flexural strength of $444 \mathrm{MPa}$.

Cersoli et al. [34] looked at how fiber volume fraction affected PLA/Kevlar composites. The volume fractions of the samples are $0,3.46,4.74$, and 20.53 percent, with the findings indicating that the greater the volume fraction, the higher the tensile strength and flexibility.

Wang et al. [109] printed composites with various fiber volume percentages, finding that the higher the fiber volume fraction, the better the mechanical characteristics. 
As demonstrated in Fig. 13 and Fig. 14, as well as the reviewed publications, raising the volume fraction improves mechanical characteristics and is a very effective factor that may boost tensile and flexural strength up to $800 \mathrm{MPa}$. Furthermore, the minimum volume fraction necessary to achieve a tensile strength greater than $600 \mathrm{MPa}$ is $25 \%$ in all but one of the articles. However, this is not the only factor that improves mechanical characteristics, and other aspects in the process parameters that will be discussed in the following sections will have an impact on this improvement.

\subsection{Printing parameters}

Layer thickness, printing speed, printing temperature, hatch spacing, and filament flow rate are all critical factors that influence mechanical qualities [102]. These five factors are nicely represented in Fig. 15. The effect of these five variables on the mechanical characteristics of composites will be discussed in the following sub-sections.

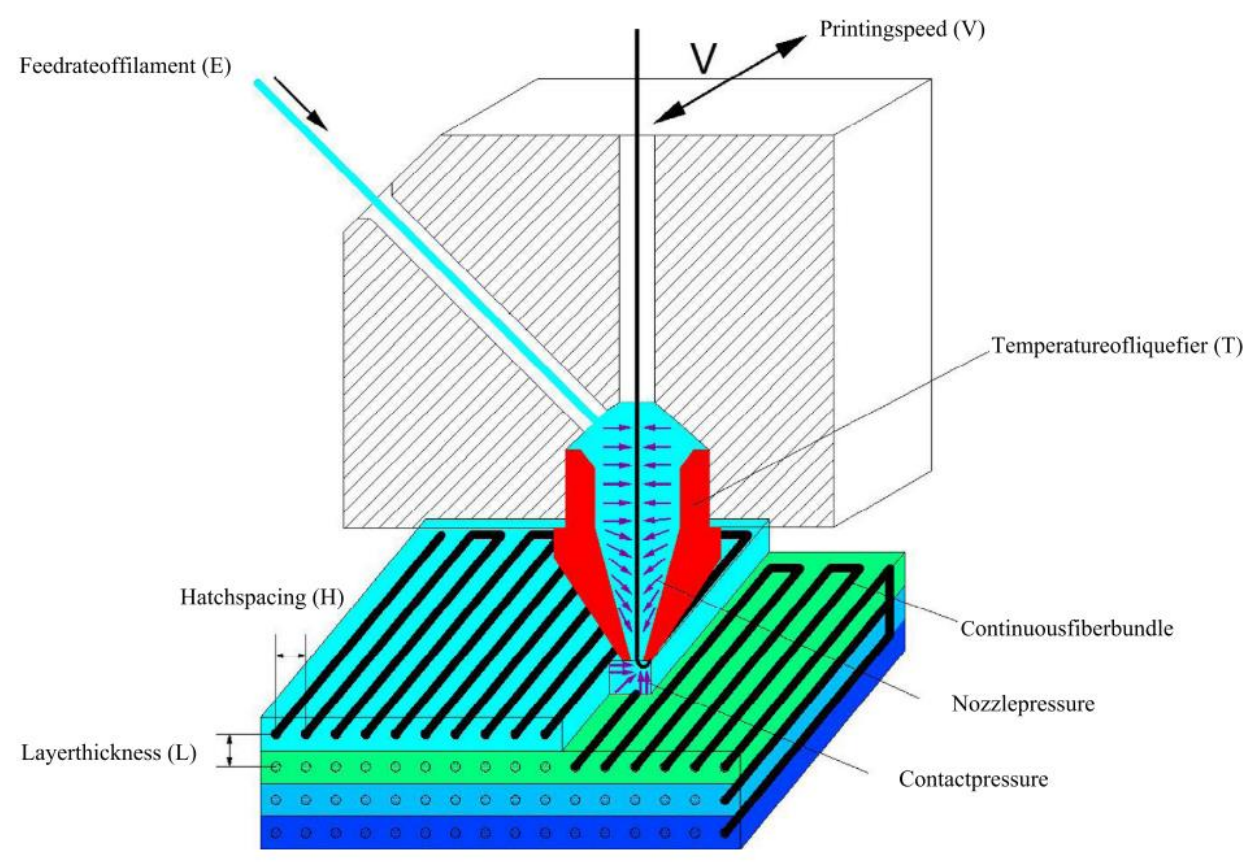

Fig. 15 Schematic of process parameters for 3D printing of CFR PLA composites [102] 


\subsubsection{Layer thickness}

The number of layers required to print the item is directly connected to the layer thickness, which in turn is directly related to the printing time. As a result, increasing layer thickness lowers manufacturing costs. Layer thickness is a key parameter in the 3D printing process, as it affects manufacturing accuracy, interfacial bonding, performance, and mechanical characteristics of manufactured samples.

Ming et al. [78] printed samples with layer thicknesses of $0.25,0.3,0.35,0.4$, and $0.45 \mathrm{~mm}$, respectively. The results indicated that when the layers are $0.4 \mathrm{~mm}$ thick, the samples' strength and flexural modulus achieve their maximum value. Because the fibers are scratched and broken when the layer thickness is less than $0.4 \mathrm{~mm}$, and when the layer thickness is greater than $0.4 \mathrm{~mm}$, the space between the layers becomes wider.

Hu et al. [56] printed composites with layer thicknesses of 0.6, 0.9, and $1.2 \mathrm{~mm}$, and found that the lower the sample thickness, the higher the flexural strength of the samples. Chacon et al. [35] printed composites with $0.1,0.125$, and $0.2 \mathrm{~mm}$ layer thicknesses. The influence of layer thickness on mechanical characteristics is minor, as evidenced by the results. Tian et al. [102] used layer thicknesses of $0.3,0.4,0.5,0.6,0.7$, and $0.8 \mathrm{~mm}$ to print composites. The findings reveal that when the thickness of the sample layer was increased, the flexural strength fell substantially, and flexural strength of $240 \mathrm{MPa}$ was reached with a layer thickness of $0.3 \mathrm{~mm}$. The flexural strength of the specimens falls somewhat as the layer thickness increases from 0.4 to $0.6 \mathrm{~mm}$, then significantly with layer thicknesses of 0.7 and $0.8 \mathrm{~mm}$. Wang et al. [109] printed samples with $0.2,0.25$, and $0.3 \mathrm{~mm}$ layer thicknesses. The results demonstrate that the mechanical characteristics improve as the layers become thinner. 
Chen et al. [37] used varied layer thicknesses of $0.5,0.6,0.7$, and $0.8 \mathrm{~mm}$ to print different samples. The authors found that increasing layer thickness lowers flexural strength and modulus, as well as impact and interlayer shear strength. The composite samples' flexural, impact, and interlayer shear strengths were the greatest at $328 \mathrm{MPa}, 155 \mathrm{~kJ} / \mathrm{m}^{2}$, and $14.0 \mathrm{MPa}$, respectively, when the layer thickness was $0.5 \mathrm{~mm}$. The layer thicknesses used by Dong et al. [42] were 0.1, 0.2, 0.3, 0.4, and $0.5 \mathrm{~mm}$. The results reveal that as the layer thickness increased, the fiber content of the samples declined significantly, from 16.32 to $3.65 \%$, as did density and tensile strength, which fell from 669 to $597.54 \mathrm{~kg} / \mathrm{m}^{3}$ and 87.36 to $27.37 \mathrm{MPa}$, respectively. The density increased by around $12 \%$ $\left(72 \mathrm{~kg} / \mathrm{m}^{3}\right)$ when the layer thickness was reduced from 0.5 to $0.1 \mathrm{~mm}$, while the tensile strength increased by $219.2 \%$ (59.99 MPa).

To summarize, the mechanical characteristics of a layer with a smaller layer thickness are superior. However, decreasing the sample thickness lengthens the printing time. As a result, an optimal thickness value must be determined that gives acceptable mechanical characteristics and a reasonable printing time.

\subsubsection{Printing speed}

Samples were printed at speeds of 200, 500, 800,1100, and $1400 \mathrm{~mm} / \mathrm{min}$ by Ming et al. [78]. The results show that when the printing speed is increased from 200 to $500 \mathrm{~mm} / \mathrm{min}$, the flexural strength and modulus remain constant. However, raising the printing speed from 500 to 1400 $\mathrm{mm} / \mathrm{min}$ considerably reduces the flexural strength.

$\mathrm{Hu}$ et al. [56] printed samples at speeds of 60,90 , and $120 \mathrm{~mm} / \mathrm{min}$. The results demonstrate that print speed has a minor impact on the samples. Printing speeds of 100, 200, 300, 400, 500, and $600 \mathrm{~mm} / \mathrm{min}$ were used by Tian et al. [102]. The effects of printing speed on the mechanical characteristics of the samples are insignificant, as shown by the results. Samples were printed at 
speeds of $2,3,4,5$, and $6 \mathrm{~mm} / \mathrm{s}$ by Chen et al. [37]. The results show that as the printing speed is increased, all of the mechanical characteristics that are evaluated decrease. Samples were printed at rates of 1,2, 3, 4, and $5 \mathrm{~mm} / \mathrm{s}$ by Wang et al. [108]. The tensile strength increases as the print speed increases then drop to a maximum of $236.7 \mathrm{MPa}$ at a speed of $3 \mathrm{~mm} / \mathrm{s}$.

Wang et al. [109] used speeds of $300,400,500,600,700$, and $800 \mathrm{~mm} / \mathrm{min}$ to print samples. The thermoplastic material may be fully connected to the bottom matrix material at printing speeds of 300,400 , and $500 \mathrm{~mm} / \mathrm{min}$. The quality of the printed corners degrades as the speed increases, but when the speed reaches 400 or $500 \mathrm{~mm} / \mathrm{min}$, the quality of the printed samples is good, and this value is also chosen for printing due to the high efficiency of $500 \mathrm{~mm} / \mathrm{min}$.

Ipekci et al. [60] printed samples at speeds of $300,600,900$, and $1200 \mathrm{~mm} / \mathrm{min}$ using photopolymer reinforced with continuous fiberglass. The results revealed that when the printing speed increases from 300 to $600 \mathrm{~mm} / \mathrm{s}$, the tensile strength improves only a little, and as the printing speed goes further, the mechanical characteristics deteriorate. As a result, $600 \mathrm{~mm} / \mathrm{s}$ was an appropriate speed for the material produced in this study.

In conclusion, the printing speed can impact the filament retention time in the extruder head as well as the resin melting rate, and if the printing speed is low, the bond between the filament and the continuous reinforcing fiber will be better. As printing speed rises, the time that filament spends in the nozzle decreases, reducing pressure and impregnation time. In most studies, increasing printing speed resulted in a loss in mechanical characteristics, whereas in others, the influence of print speed on mechanical properties was shown to be insignificant. 


\subsubsection{Printing temperature}

Temperature is a significant factor in composites manufacturing because it influences the impregnation of reinforcing fibers and matrices. The melting temperature in the printer head is also crucial for bonding printed pathways and layers in the 3D printing process. Hu et al. [56] printed samples at 200, 215, and 230 degrees Celsius. The results demonstrate that temperature has a negligible influence on the samples.

Printing samples at $180,190,200,210,220,230$, and $240{ }^{\circ} \mathrm{C}$ was done by Tian et al. [102]. The results show that as the temperature rises, the flexural strength and modulus increase, with the flexural strength and modulus at $240^{\circ} \mathrm{C}$ being $155 \mathrm{MPa}$ and $8.6 \mathrm{GPa}$, respectively. The sample produced at $240^{\circ} \mathrm{C}$, on the other hand, lost its surface precision due to PLA melt overflow. As a result, the printer's maximum suggested temperature is $230^{\circ} \mathrm{C}$, at which the produced composite samples' flexural strength and modulus were $145 \mathrm{MPa}$ and 8.6 MPa, respectively. The ideal process temperature was between 200 and 230 degrees Celsius.

Chen et al. [37] printed samples at temperatures of 190, 200, 210, 220, and 230 degrees Celsius. According to the findings, boosting the temperature enhanced all of the studied mechanical characteristics. However, when the printing temperature surpasses $210^{\circ} \mathrm{C}$, it becomes difficult to maintain the sample's dimensional stability, particularly at the edges. As a result, $210^{\circ} \mathrm{C}$ has been chosen as the optimal printing temperature.

Wang et al. [108] printed samples at temperatures of 255, 265, 275, and 285 degrees Celsius. The tensile strength of samples printed at 265 and $275^{\circ} \mathrm{C}$ was equivalent and maximal, according to the data. These two samples have tensile strengths of 236.7 and $233.4 \mathrm{MPa}$, respectively. Furthermore, at $265^{\circ} \mathrm{C}$, the tensile modulus reached a high of $33.15 \mathrm{GPa}$. Because of the visible flaws, the composite produced at $285^{\circ} \mathrm{C}$ is regarded as less desirable. 
Wang et al. [109] printed composites at 170, 180, 190, 200, 210, and 220 degrees Celsius. The composite printed at $210^{\circ} \mathrm{C}$ had the best aesthetic and quality attributes, according to the results.

In conclusion, when the printing temperature rises, so do the mechanical characteristics. Because the molten filament forms a stronger connection with the produced composite as the printing temperature rises. However, printed composites lose their aesthetic characteristics and dimensional precision at extremely high temperatures. As a result, a temperature should be chosen that preserves the part's appearance and dimensional accuracy while still providing acceptable mechanical characteristics.

\subsubsection{Hatch spacing}

Tian et al. [102] used hatch spacing of $0.4,0.6,0.8,1,1.2,1.4,1.6$, and $1.8 \mathrm{~mm}$ to print composites. The average flexural strength increased from 130 to $335 \mathrm{MPa}$ and the flexural modulus improved from 6.26 to $30 \mathrm{~GB}$ when hatch spacing was reduced from 1.8 to $0.4 \mathrm{~mm}$.

\subsubsection{Feed rate of the filament}

The filament feed rate is proportional to the amount of material supplied into the printing head per unit volume. The feed rate of the filament determines the inner pressure and extrusion speed of melt material via the printing head when the tip diameter of the extrusion nozzle is the same [102].

At rates of 30,55, 70, and $80 \mathrm{~mm} / \mathrm{min}$, Liu et al. [71] fed filaments into the 3D printer's extruder head. The flexural strength of the sample fed at a rate of $55 \mathrm{~mm} / \mathrm{min}$ rose by $15 \%$ when compared to the sample fed at a rate of $70 \mathrm{~mm} / \mathrm{min}$, resulting in flexural strength of $504.58 \mathrm{MPa}$ for the sample fed at $50 \mathrm{~mm} / \mathrm{min}$ and $437.76 \mathrm{MPa}$ for the sample fed at $70 \mathrm{~mm} / \mathrm{min}$. When the feed rate is set to $30 \mathrm{~mm} / \mathrm{min}$, however, the enhancing impact decreases because the limited amount of resin materials used resulted in low forming pressure and poor interfacial performance. The sample fed 
at $80 \mathrm{~mm} / \mathrm{min}$ had nearly the same flexural strength as the sample fed at $70 \mathrm{~mm} / \mathrm{min}$. Furthermore, no improvement occurred because the forming pressure was so high that overfilled resin materials caused the interfacial performance to become excessively strong, resulting in brittle fracture.

Feed rates of $60,80,100,120,140$, and $160 \mathrm{~mm} / \mathrm{min}$ were employed by Tian et al. [102]. The results showed that raising the feeding speed from 60 to $80 \mathrm{~mm} / \mathrm{min}$ greatly increased flexural strength. Due to the large unit volume of extruded materials, which formed the dual interfaces between fiber and matrix, as well as deposited lines in the 3D printed CFR composites. This could be caused by an increase in inner pressure in the liquefier and overlapping contact pressure between adjacent deposited lines. However, as the feed rate was increased, the flexural strength did not improve, most likely due to the short fiber impregnation period.

In summary, mechanical properties improve as the feed rate of the filament increases to some extent. The mechanical properties remain constant as the feed rate of the filament increases further. More study, however, is required to better understand the influence of feed rate on the mechanical characteristics of CFRCs.

\subsubsection{Fiber orientation}

The fibers can be printed in two ways, as shown in Fig. 16, concentric and isotropic. They can be printed at varied angles $(0, \pm 45,90$ degrees, see Fig. 17) in the isotropic form. 


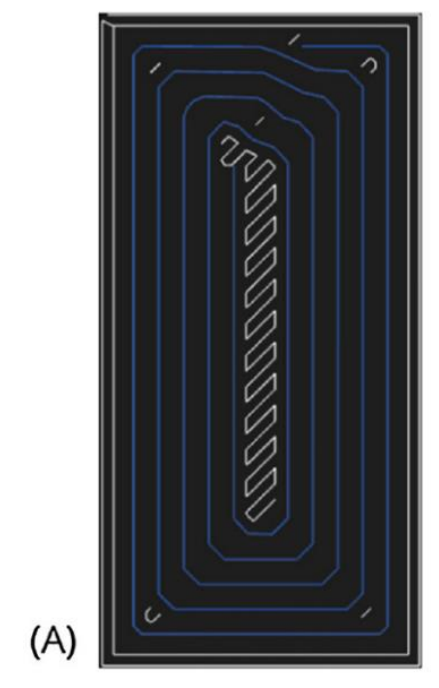

(B)

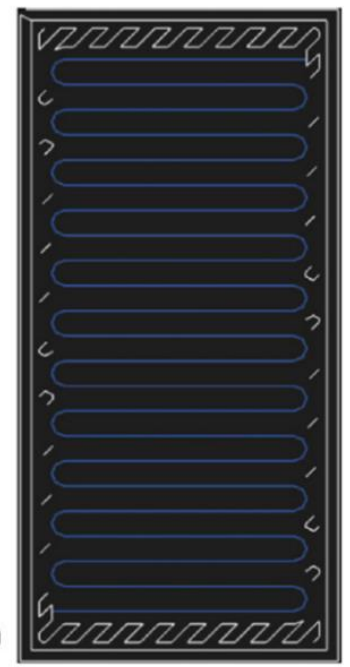

Fig. 16 Fiber reinforcement configuration, (A) concentric, and (B) isotropic - 0 degree [29]

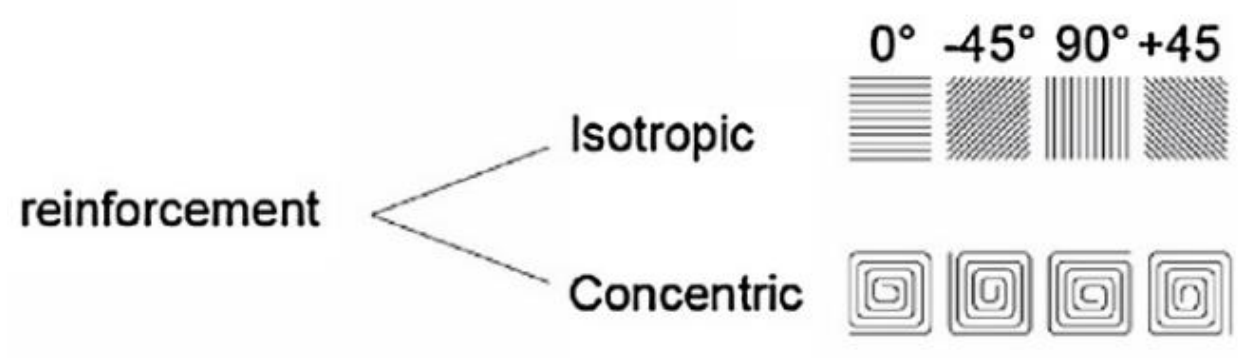

Fig. 17 Fiber orientations in a single layer [29]

In printing nylon/CF composites, Pyl et al. [91] adjusted the fiber orientations of the composites to 0 unidirectional, $0 / 90,0 / 90 / \pm 45$, and \pm 45 degrees. According to the findings, composites with the fiber orientations of 0 unidirectional, $0 / 90,0 / 90 / \pm 45$, and \pm 45 degrees had the highest tensile strength in that order. The strain to failure of the composites printed at 0 unidirectional, $0 / 90 / \pm 45$ degrees was nearly identical, but the strain to failure of the \pm 45 degrees sample was about four times that of the others. 
Printed nylon matrix composites reinforced with carbon, glass, and Kevlar fibers were produced by Mohammadizadeh et al. [80]. The samples' infill pattern was rectangular, and their fill density was $50 \%$. In addition, the sample infill orientations were concentric and isotropic. The composites produced in an isotropic configuration showed greater mechanical qualities than those printed in a concentric configuration, according to the findings.

Concentric and isotropic infill arrangements were employed by Yu et al. [113]. Composites with a concentric infill pattern offered higher flexural strength and energy absorption than composites with an isotropic infill pattern.

Araya-Calvo et al. [29] used concentric and isotropic infill patterns to print PA6/CF composites. Compressive and flexural tests were performed. The results of these experiments revealed that composites with a concentric pattern performed better. 1.69 GPa, 40.5 MPa, 59.07 MPa, and 5.41 GPa, respectively, were the compressive modulus, proportional limit, flexural strength, and flexural modulus of these composites.

Dickson et al. [39] used carbon, glass, and Kevlar fibers to reinforce nylon matrix composites. Concentric and isotropic designs were used to print the fibers. The composites with isotropic patterns had higher tensile and flexural strength and modulus than concentric specimens. In addition, the flexural testing revealed that the concentric pattern composites perform better in bending than in tension.

Gonzalez-Estrada et al. [50] used angles of 0, 45, and 90 degrees to print nylon/GF composites. The composites printed at 0,45 , and 90 degrees had the highest strength and modulus, respectively. When compared to the 45 degree sample, the 0 degree sample has significantly better strength and modulus. Nylon/Kevlar composites with angles of 30, 45, and 60 degrees were created by Shi et 
al. [97]. The lower the angle, the greater the yield stress, i.e., the highest yield stress is for 30, 45, and 60 degrees, respectively.

In conclusion, increasing the print orientation (from 0 to 90 degrees) reduces the mechanical characteristics of composites produced with the isotropic pattern. The orientation of the reinforcing fibers, on the other hand, must be 0 degrees to have high mechanical qualities. It is impossible to determine which of the isotropic and concentric patterns has higher mechanical qualities. Because in some circumstances $[39,80]$, an isotropic pattern gave higher mechanical qualities, whereas, in other studies [29, 113], concentric patterns were found to produce better mechanical properties.

\subsubsection{Build orientation}

The mechanical qualities of printed composites are also influenced by the build orientation. The composites can be built in one of three orientations, as shown in Fig. 18: flat, on-edge, and upright. Chacon et al. [35] tested nylon matrix composites reinforced with carbon, Kevlar, and glass fibers

in Charpy tests. The composites were printed in two orientations: flat and on-edge (the fill density and fill pattern were 100 percent and rectangular and isotropic with zero degrees angle). The onedge composites had higher impact strength, according to the findings. 


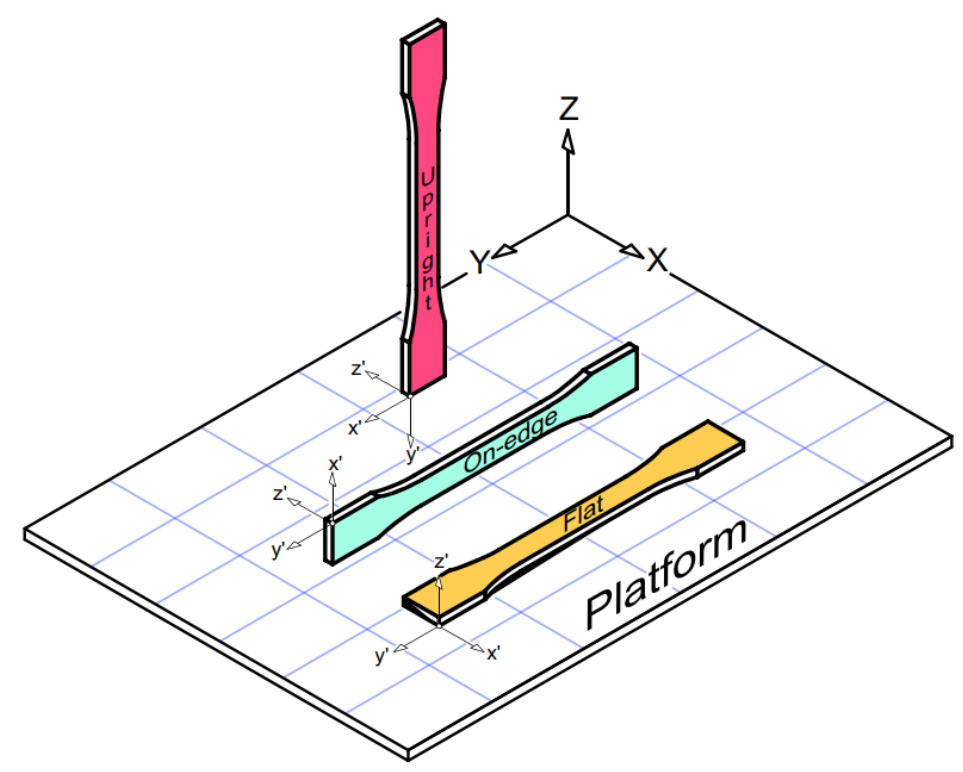

Fig. 18 Different types of build orientation [117]

\subsubsection{Infill pattern \& density}

Naranjo-Lozada et al. [84] created Onyx/CF composites with infill densities of $10 \%$ and $70 \%$ with triangular and rectangular infill patterns. Composites with a triangle design performed better in tensile tests than those with a rectangular pattern. The modulus of elasticity improved when the infill density of the specimens was increased from $10 \%$ to $70 \%$, but the tensile yield strength of these two composites was nearly identical.

Gonzalez-Estrada et al. [50] printed nylon/GF and nylon/CF composites with triangular, rectangular, and hexagonal infill patterns. The samples' infill density was $20 \%$ and $50 \%$, respectively. Higher stiffness and ultimate tensile strength were achieved with the triangular pattern. Changing the infill density from $20 \%$ to $50 \%$, on the other hand, has no noticeable impact on the properties indicated. Increasing the infill density from $20 \%$ to $50 \%$ resulted in a 3.3 percent improvement in elastic modulus and a 5.5 percent increase in tensile strength. 
Printed nylon matrix composite reinforced with carbon, glass, and Kevlar fibers by Mei et al. [75]. The composite's infill patterns were rectangular, hexagonal, and triangular. The samples had the same number of concentric fiber rings and fiber layers. Rectangular infill had values of 4, 6, and 8; hexagonal infill had a value of 6 , and triangular infill had a value of 6 . The rectangular structure with 8 concentric fiber rings and fiber layers had the highest tensile strength and modulus, according to the data. As a result, the tensile strength and modulus increase as the number of concentric fiber rings and fiber layers increases.

According to the articles listed above, increasing the amount of infill density improves mechanical qualities slightly. In addition, the triangular, hexagonal, and rectangular infill patterns, respectively, offered the best mechanical qualities. The explanation for the rectangular sample's superiority in Mei et al. [75] is that the analyzed samples had more rims than the other samples.

\subsection{Compaction roller technique}

Ueda et al. [27] created two samples, one with compaction rollers and the other with the hotpressing method. The compression roller (see Fig. 5 (d)) and the printer bed had temperatures of $270^{\circ} \mathrm{C}$ and $100^{\circ} \mathrm{C}$, respectively. The results demonstrated that the compaction roller improves the printed composite's mechanical properties, with the printed composite having a better fracture surface than the hot press sample. The roller-made composite had a smoother surface than the hotpressed one. The roller-made sample had a void fraction of 3\%, while the hot press sample had a void fraction of $10 \%$.

Zhang et al. [115] printed PLA/CF composites with a compaction roller (see Fig. 19). The sample's tensile and flexural strength rose with increasing pressure to $644.8 \mathrm{MPa}$ and $401.24 \mathrm{MPa}$, respectively (compared to the tensile and flexural strength of specimens without pressure, which 
was 109.9 $\mathrm{MPa}$ and 163.13 $\mathrm{MPa}$, respectively). Furthermore, as the pressure is increased, the quality of the sample surface degrades, and the sample may fail.

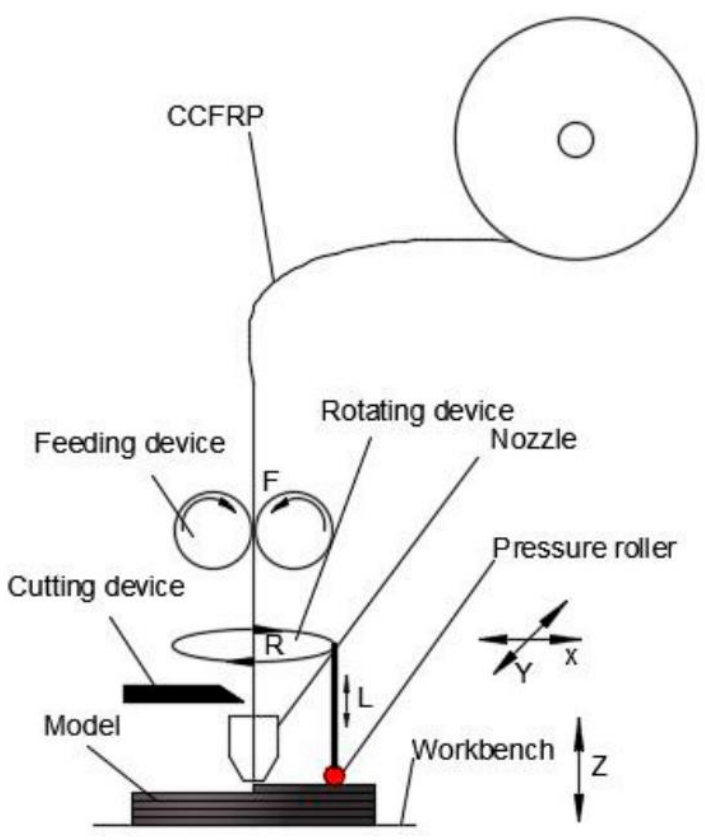

Fig. 19 Schematic of the 3D-printing machine. [115]

Based on the findings of $[27,115]$, it can be deduced that applying pressure during printing improves the mechanical properties greatly. Furthermore, this process has capabilities that are comparable to the hot press method.

\section{Postprocessing}

Postprocessing is one of the most effective approaches to improve the mechanical properties of CFRCs. Three of the four articles that performed postprocessing on their samples acquired extraordinarily high tensile and flexural strength, as shown in Table 3. The number of publications 
devoted to postprocessing processes, on the other hand, is quite minimal (see

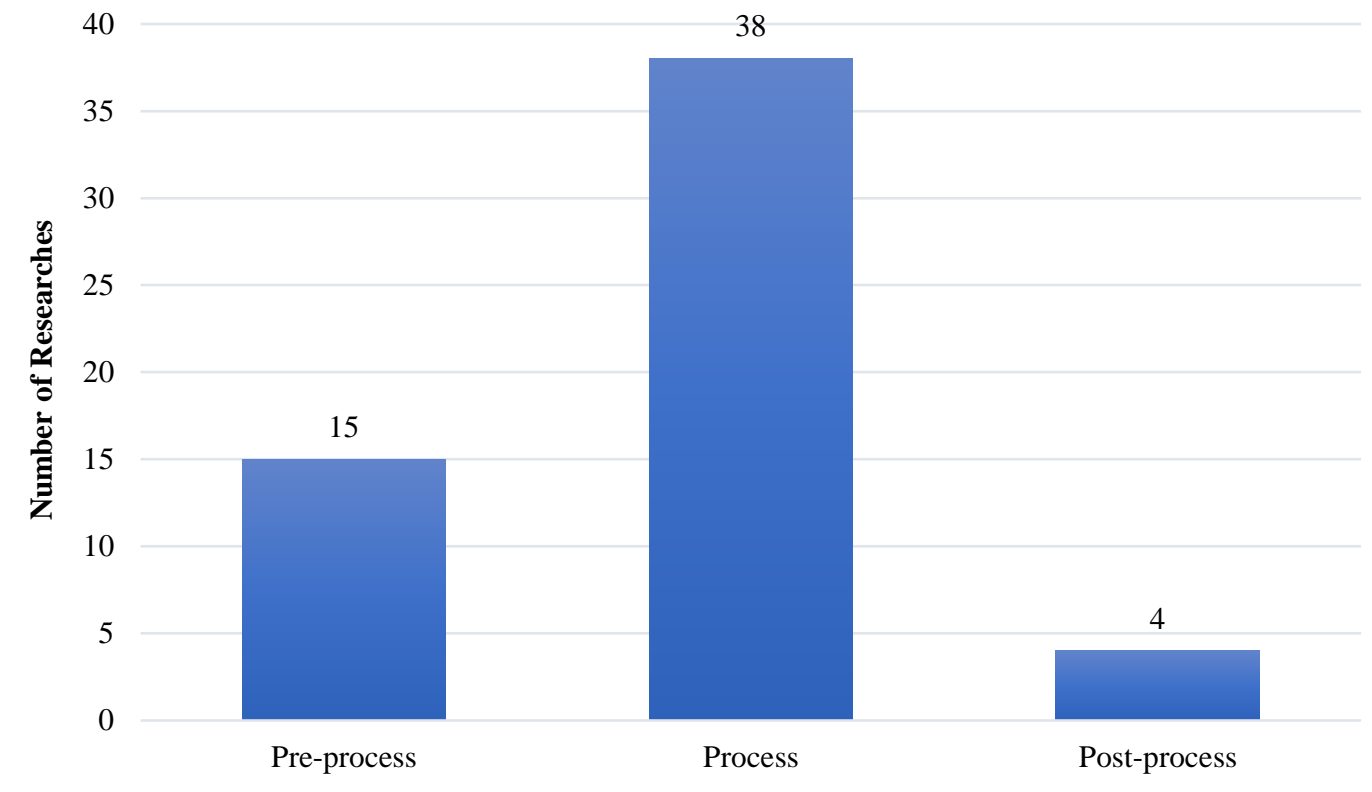

Fig. 7). The high expenses of postprocessing activities could be one cause for this. There is a need for more research in this area, as well as the application of postprocessing to improve mechanical qualities in the future as technology advances. In what follows, a brief review of articles dedicated to CFRC postprocessing will be discussed.

As indicated in the previous section, Ueda et al. [27] used a compaction roller to make composites (see Fig. 5(d)). They also hot-pressed 3D-printed composites for 10 minutes at a temperature of $230^{\circ} \mathrm{C}$ and a pressure of $0.1 \mathrm{MPa}$ (see Fig. 20). The sample thickness was reduced by 16 percent and the weight was reduced by 1.5 percent after hot pressing. The flexural properties of this sample were comparable to those of a compaction roller-produced sample. It can be concluded that thermal compaction rollers can be employed instead of hot pressing. 

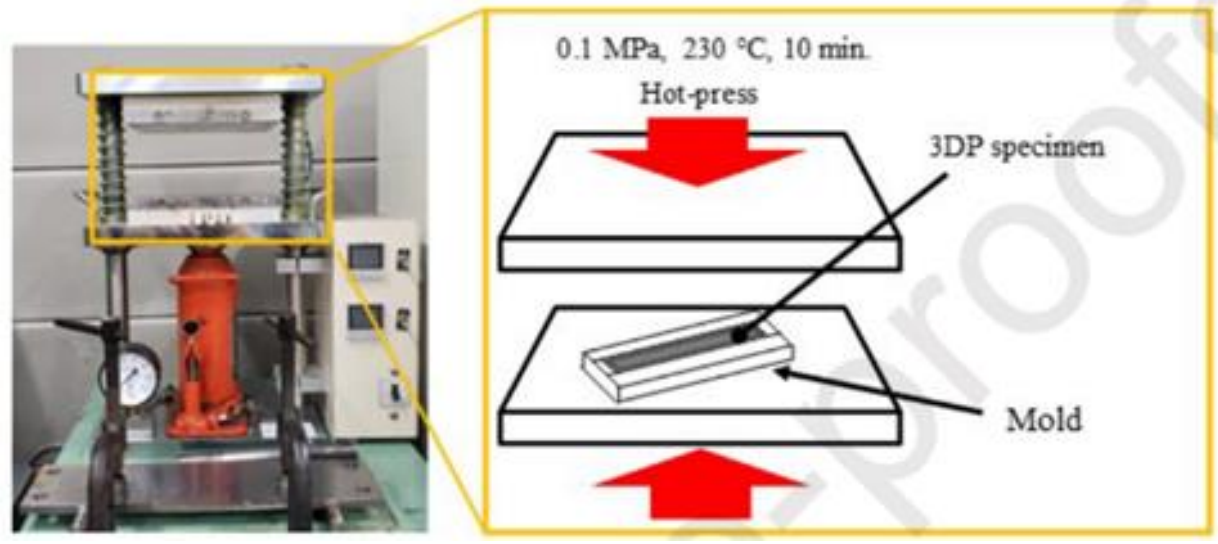

Fig. 20 Hot-press operation on reinforced composites [27]

Ming et al. [78] used a vacuum chamber to cure 3D printed materials at various curing pressures and temperatures (see Fig. 11 (a)). The specimens were first exposed to vacuum pressures of -10 , $-30,-50,-70$, and $-90 \mathrm{kPa}$. The results revealed that the higher the vacuum pressure (the more negative the pressure), the better the specimens' strength and flexural modulus. The fibers were discovered to be covered with a thin layer of resin before curing, and there were several voids inside the specimens. But after curing, the fibers were evenly covered with resin, and the interior voids were significantly reduced. Negative pressure can increase resin diffusion and fiber impregnation, according to these findings. A small pressure increase led to a significant decrease in fiber content. As a result, because it affects resin flow, void distribution, and fiber content during curing, curing pressure is a vital parameter that must be regulated. Curing temperatures of 150 , 160, 170, 180, and 190 degrees Celsius were also applied to the samples. The results revealed that increasing the curing temperature from 150 to 170 degrees enhanced flexural strength and modulus to their maximum levels. On the other hand, the flexural strength and modulus decreased dramatically as the curing temperature increased from 170 to 190 degrees Celsius. 
Hot pressing and vacuuming are two feasible options for postprocessing and thereby increasing the mechanical properties of CFRCs, according to the findings of these two papers. The higher negative the pressure in a vacuum system, the better the mechanical properties.

\section{Suggestions}

According to the previous sections' comments, a lot of topics in CFRC 3D printing are either unexplored or underexplored. These themes could be interesting and relevant research subjects in the future:

1. CFRCs have yet to be subjected to wear and friction tests.

2. A modest number of studies have been conducted on CFRC dynamic, fatigue, creep, impact, and indentation tests.

3. The impregnation and postprocessing activities should be given special attention. In addition, more research is needed to understand the impact of postprocessing factors on composite mechanical properties.

4. More research might be done on build orientation, such as printing specimens flat, on-edge, and upright, and investigating the effects on mechanical qualities.

5. The hatch spacing process parameter should be adjusted, and the impacts on mechanical qualities should be investigated.

6. More research on isotropic and concentric printed samples is needed.

7. Kevlar and glass fiber reinforced composites' bending characteristics should be investigated further. The impact of fiber volume fraction on the bending strength of these composites should be studied in particular.

8. It is necessary to evaluate the impact of specimen geometry, printing parameters, and printing patterns on printing time and specimen defects. 
9. The ability to undertake post-printing procedures such as forming and machining, as well as the consequences of these operations on the features of 3D printed CFRCs, is further intriguing topics for wider use of these composites.

\section{Conclusions}

The majority of CFRC research employing FDM technology has been covered in this review paper. All of the parameters impacting the mechanical properties of CFRCs were evaluated. The following are the conclusions reached:

Impregnation is critical in pre-processing, and the results reveal that the higher the impregnation quality, the better the mechanical qualities. Pre-heating the fiber, applying ultrasonic vibration, and pre-impregnation inside a solution all help to increase impregnation and, as a result, improve mechanical qualities.

The following findings are drawn from examining the process (printing) parameters:

- Tensile and flexural strength and modulus rise as the fiber volume percentage increases.

- The thinner the layer thickness, the greater the mechanical qualities.

- As printing speed drops, mechanical qualities improve.

- The mechanical properties of the samples improve as the printing temperature rises, but it should be noted that as the print temperature rises, the appearance properties and dimensional precision of the samples deteriorate.

- As the quantity of hatch spacing is reduced, mechanical qualities improve.

- As the filament feed rate is raised, the mechanical characteristics improve initially and then remain constant. 
- For specimens having an isotropic pattern printed on them, the lower the mechanical qualities, the larger the angle of the fibers ( 0 degrees provides the best mechanical properties).

- The samples with a higher percentage of infill density have slightly better mechanical properties.

- The triangular, hexagonal, and rectangular infill patterns have the best mechanical properties, respectively.

- The use of hot compaction rollers will greatly improve mechanical qualities, but extreme caution must be exercised to avoid over-pressuring the specimens.

Finally, proven postprocessing methods for improving the mechanical properties of CFRCs include hot pressing, vacuuming, and post-heat treatment.

\section{References}

1. Mazurchevici, A.D., D. Nedelcu, and R. Popa, Additive manufacturing of composite materials by FDM technology: A review. Indian Journal of Engineering and Materials Sciences (IJEMS), 2021. 27(2): p. 179-192.

2. Joshi, S.C. and A.A. Sheikh, 3D printing in aerospace and its long-term sustainability. Virtual and Physical Prototyping, 2015. 10(4): p. 175-185.

3. Lim, C.W.J., et al., An overview of 3-D printing in manufacturing, aerospace, and automotive industries. IEEE potentials, 2016. 35(4): p. 18-22. 
4. Aimar, A., A. Palermo, and B. Innocenti, The role of $3 D$ printing in medical applications: $a$ state of the art. Journal of healthcare engineering, 2019. 2019.

5. Ventola, C.L., Medical applications for 3D printing: current and projected uses. Pharmacy and Therapeutics, 2014. 39(10): p. 704.

6. Cai, H., Application of 3D printing in orthopedics: status quo and opportunities in China. Annals of translational medicine, 2015. 3(Suppl 1).

7. Nicholas, P., et al., Integrating real-time multi-resolution scanning and machine learning for Conformal Robotic 3D Printing in Architecture. International Journal of Architectural Computing, 2020. 18(4): p. 371-384.

8. Mathur, R., 3D printing in architecture. International Journal of Innovative Science, Engineering \& Technology, 2016. 3(7): p. 583-591.

9. Balletti, C., M. Ballarin, and F. Guerra, 3D printing: State of the art and future perspectives. Journal of Cultural Heritage, 2017. 26: p. 172-182.

10. Liu, Z., et al., 3D printing: Printing precision and application in food sector. Trends in Food Science \& Technology, 2017. 69: p. 83-94.

11. Derossi, A., et al., Application of 3D printing for customized food. A case on the development of a fruit-based snack for children. Journal of Food Engineering, 2018. 220: p. 6575.

12. Godoi, F.C., S. Prakash, and B.R. Bhandari, 3d printing technologies applied for food design: Status and prospects. Journal of Food Engineering, 2016. 179: p. 44-54.

13. Vanderploeg, A., S.-E. Lee, and M. Mamp, The application of $3 D$ printing technology in the fashion industry. International Journal of Fashion Design, Technology and Education, 2017. 10(2): p. 170-179. 
14. Spahiu, T., et al. On the possible use of $3 D$ printing for clothing and shoe manufacture. in Proceedings of the 7th International Conference of Textile, Tirana, Albania. 2016.

15. Spahiu, T., E. Canaj, and E. Shehi, 3D printing for clothing production. Journal of Engineered Fibers and Fabrics, 2020. 15: p. 1558925020948216.

16. Bikas, H., P. Stavropoulos, and G. Chryssolouris, Additive manufacturing methods and modelling approaches: a critical review. The International Journal of Advanced Manufacturing Technology, 2016. 83(1-4): p. 389-405.

17. Akhoundi, B., A.H. Behravesh, and A. Bagheri Saed, Improving mechanical properties of continuous fiber-reinforced thermoplastic composites produced by FDM $3 D$ printer. Journal of Reinforced Plastics and Composites, 2019. 38(3): p. 99-116.

18. Al Abadi, H., et al., Elastic properties of 3D printed fibre-reinforced structures. Composite Structures, 2018. 193: p. 8-18.

19. Arif, M., et al., Performance of biocompatible PEEK processed by fused deposition additive manufacturing. Materials \& Design, 2018. 146: p. 249-259.

20. Wang, X., et al., 3D printing of polymer matrix composites: A review and prospective. Composites Part B: Engineering, 2017. 110: p. 442-458.

21. Kabir, S.F., K. Mathur, and A.-F.M. Seyam, A critical review on 3D printed continuous fiber-reinforced composites: History, mechanism, materials and properties. Composite Structures, 2020. 232: p. 111476.

22. Crump, S., Apparatus and method for creating three-dimensional objects (US Patent US5121329A). 1989.

23. Bryll, K., et al. Polymer composite manufacturing by FDM 3D printing technology. in MATEC Web of Conferences. 2018. EDP Sciences. 
24. Sanei, S.H.R. and D. Popescu, 3D-Printed carbon fiber reinforced polymer composites: $a$ systematic review. Journal of Composites Science, 2020. 4(3): p. 98.

25. Wickramasinghe, S., T. Do, and P. Tran, FDM-based $3 D$ printing of polymer and associated composite: A review on mechanical properties, defects and treatments. Polymers, 2020. 12(7): p. 1529.

26. Shang, J., et al., Controllable inter-line bonding performance and fracture patterns of continuous fiber reinforced composites by sinusoidal-path $3 D$ printing. Composites Science and Technology, 2020. 192: p. 108096.

27. Ueda, M., et al., 3D compaction printing of a continuous carbon fiber reinforced thermoplastic. Composites Part A: Applied Science and Manufacturing, 2020. 137: p. 105985.

28. Akhoundi, B., A.H. Behravesh, and A. Bagheri Saed, An innovative design approach in three-dimensional printing of continuous fiber-reinforced thermoplastic composites via fused deposition modeling process: in-melt simultaneous impregnation. Proceedings of the Institution of Mechanical Engineers, Part B: Journal of Engineering Manufacture, 2020. 234(1-2): p. 243259.

29. Araya-Calvo, M., et al., Evaluation of compressive and flexural properties of continuous fiber fabrication additive manufacturing technology. Additive Manufacturing, 2018. 22: p. 157164.

30. Vinoth Babu, N., et al., Influence of slicing parameters on surface quality and mechanical properties of 3D-printed CF/PLA composites fabricated by FDM technique. Materials Technology, 2021: p. 1-18.

31. Baumann, F., J. Scholz, and J. Fleischer, Investigation of a new approach for additively manufactured continuous fiber-reinforced polymers. Procedia Cirp, 2017. 66: p. 323-328. 
32. Bettini, P., et al., Fused deposition technique for continuous fiber reinforced thermoplastic. Journal of Materials Engineering and Performance, 2017. 26(2): p. 843-848.

33. Caminero, M., et al., Impact damage resistance of $3 D$ printed continuous fibre reinforced thermoplastic composites using fused deposition modelling. Composites Part B: Engineering, 2018. 148: p. 93-103.

34. Cersoli, T., et al., 3D printing of a continuous fiber-reinforced composite based on a coaxial Kevlar/PLA filament. Composites and Advanced Materials, 2021. 30: p. 26349833211000058.

35. Chacón, J., et al., Additive manufacturing of continuous fibre reinforced thermoplastic composites using fused deposition modelling: Effect of process parameters on mechanical properties. Composites science and technology, 2019. 181: p. 107688.

36. Chaudhry, F.N., et al., Effect of carbon fibre on reinforcement of thermoplastics using FDM and RSM. Journal of Thermoplastic Composite Materials, 2019: p. 0892705719886891.

37. Chen, K., et al., Optimization of printing parameters of 3D-printed continuous glass fiber reinforced polylactic acid composites. Thin-Walled Structures, 2021. 164: p. 107717.

38. Chen, W., et al., Process evaluation, tensile properties, mathematical models, and fracture behavior of $3 D$ printed continuous fiber reinforced thermoplastic composites. Journal of Reinforced Plastics and Composites, 2021: p. 07316844211016091.

39. Dickson, A.N., et al., Fabrication of continuous carbon, glass and Kevlar fibre reinforced polymer composites using additive manufacturing. Additive Manufacturing, 2017. 16: p. 146152. 
40. Dikshit, V., et al., Quasi-static indentation analysis on three-dimensional printed continuous-fiber sandwich composites. Journal of Sandwich Structures \& Materials, 2019: p. 1099636219836058.

41. Dong, G., et al., Mechanical properties of continuous kevlar fiber reinforced composites fabricated by fused deposition modeling process. Procedia Manufacturing, 2018. 26: p. 774781.

42. Dong, K., et al., Mechanical properties and shape memory effect of 4D printed cellular structure composite with a novel continuous fiber-reinforced printing path. Materials \& Design, 2021. 198: p. 109303.

43. Dong, K., et al., 3D printing of continuous fiber reinforced diamond cellular structural composites and tensile properties. Composite Structures, 2020. 250: p. 112610.

44. Dugbenoo, E., et al., Enhanced Bonding via Additive Manufacturing-Enabled Surface Tailoring of 3D Printed Continuous-Fiber Composites. Advanced Engineering Materials, 2018. 20(12): p. 1800691.

45. Dutra, T.A., et al., Mechanical characterization and asymptotic homogenization of 3Dprinted continuous carbon fiber-reinforced thermoplastic. Journal of the Brazilian Society of Mechanical Sciences and Engineering, 2019. 41(3): p. 1-15.

46. Fernandes, R.R., A.Y. Tamijani, and M. Al-Haik, Mechanical characterization of additively manufactured fiber-reinforced composites. Aerospace Science and Technology, 2021. 113: p. 106653.

47. Ghebretinsae, F., O. Mikkelsen, and A. Akessa. Strength analysis of 3D printed carbon fibre reinforced thermoplastic using experimental and numerical methods. in IOP Conference Series: Materials Science and Engineering. 2019. IOP Publishing. 
48. Giannakis, E., et al., Static and fatigue properties of $3 D$ printed continuous carbon fiber nylon composites. Int. J. Mod. Manuf. Technol, 2019. 11: p. 69-76.

49. Goh, G.D., et al., Characterization of mechanical properties and fracture mode of additively manufactured carbon fiber and glass fiber reinforced thermoplastics. Materials \& Design, 2018. 137: p. 79-89.

50. González-Estrada, O.A., A. Pertuz, and J.E. Quiroga Mendez. Evaluation of tensile properties and damage of continuous fibre reinforced 3D-printed parts. in Key Engineering Materials. 2018. Trans Tech Publ.

51. Hao, W., et al., Preparation and characterization of 3D printed continuous carbon fiber reinforced thermosetting composites. Polymer Testing, 2018. 65: p. 29-34.

52. Hedayati, S.K., et al., 3D printed PCL scaffold reinforced with continuous biodegradable fiber yarn: A study on mechanical and cell viability properties. Polymer Testing, 2020. 83: p. 106347.

53. Heidari-Rarani, M., M. Rafiee-Afarani, and A. Zahedi, Mechanical characterization of FDM 3D printing of continuous carbon fiber reinforced PLA composites. Composites Part B: Engineering, 2019. 175: p. 107147.

54. Hetrick, D.R., et al., Charpy impact energy absorption of $3 D$ printed continuous Kevlar reinforced composites. Journal of Composite Materials: p. 0021998320985596.

55. Hou, Z., et al., A constitutive model for $3 D$ printed continuous fiber reinforced composite structures with variable fiber content. Composites Part B: Engineering, 2020. 189: p. 107893.

56. $\mathrm{Hu}, \mathrm{Q}$., et al., Manufacturing and $3 D$ printing of continuous carbon fiber prepreg filament. Journal of materials science, 2018. 53(3): p. 1887-1898. 
57. $\mathrm{Hu}, \mathrm{Y}$., et al., Carbon fibre damage during $3 d$ printing of polymer matrix laminates using the fdm process. Materials \& Design, 2021: p. 109679.

58. Ibrahim, Y., et al., Effective thermal conductivity of 3D-printed continuous fiber polymer composites. Advanced Manufacturing: Polymer \& Composites Science, 2020. 6(1): p. 17-28.

59. Imeri, A., et al., Fatigue analysis of the fiber reinforced additively manufactured objects. The International Journal of Advanced Manufacturing Technology, 2018. 98(9): p. 2717-2724. 60. İpekçi, A. and B. Ekici, Experimental and statistical analysis of robotic 3D printing process parameters for continuous fiber reinforced composites. Journal of Composite Materials, 2021: p. 0021998321996425.

61. Iragi, M., et al., Ply and interlaminar behaviours of 3D printed continuous carbon fibrereinforced thermoplastic laminates; effects of processing conditions and microstructure. Additive Manufacturing, 2019. 30: p. 100884.

62. Ishii, K., et al., Bending fracture rule for 3D-printed curved continuous-fiber composite. Advanced Composite Materials, 2018.

63. Jahangir, M., et al., Reinforcement of material extrusion 3D printed polycarbonate using continuous carbon fiber. Additive Manufacturing, 2019. 28: p. 354-364.

64. Justo, J., et al., Characterization of $3 D$ printed long fibre reinforced composites. Composite Structures, 2018. 185: p. 537-548.

65. Kabir, S.F., K. Mathur, and A.-F.M. Seyam, Impact resistance and failure mechanism of $3 D$ printed continuous fiber-reinforced cellular composites. The Journal of The Textile Institute, 2020: p. 1-15. 
66. Kousiatza, C., D. Tzetzis, and D. Karalekas, In-situ characterization of $3 D$ printed continuous fiber reinforced composites: A methodological study using fiber Bragg grating sensors. Composites Science and Technology, 2019. 174: p. 134-141.

67. Li, H., et al., The quantitative analysis of tensile strength of additively manufactured continuous carbon fiber reinforced polylactic acid (PLA). Rapid Prototyping Journal, 2019.

68. Li, N., Y. Li, and S. Liu, Rapid prototyping of continuous carbon fiber reinforced polylactic acid composites by 3D printing. Journal of Materials Processing Technology, 2016. 238: p. 218-225.

69. Liu, Z., J. Shi, and Y. Wang. Evaluating Tensile Properties of 3D Printed Continuous Fiber Reinforced Nylon 6 Nanocomposites. in International Manufacturing Science and Engineering Conference. 2018. American Society of Mechanical Engineers.

70. Liu, S., Y. Li, and N. Li, A novel free-hanging 3D printing method for continuous carbon fiber reinforced thermoplastic lattice truss core structures. Materials \& Design, 2018. 137: p. 235-244.

71. Liu, T., et al., Interfacial performance and fracture patterns of $3 D$ printed continuous carbon fiber with sizing reinforced PA6 composites. Composites Part A: Applied Science and Manufacturing, 2018. 114: p. 368-376.

72. Luan, C., et al., Large-scale deformation and damage detection of $3 D$ printed continuous carbon fiber reinforced polymer-matrix composite structures. Composite Structures, 2019. 212: p. $552-560$.

73. Luo, H., et al., Selectively enhanced $3 D$ printing process and performance analysis of continuous carbon fiber composite material. Materials, 2019. 12(21): p. 3529. 
74. Matsuzaki, R., et al., Three-dimensional printing of continuous-fiber composites by innozzle impregnation. Scientific reports, 2016. 6(1): p. 1-7.

75. Mei, H., et al., Tailoring strength and modulus by $3 D$ printing different continuous fibers and filled structures into composites. Advanced Composites and Hybrid Materials, 2019. 2(2): p. 312-319.

76. Melenka, G.W., et al., Evaluation and prediction of the tensile properties of continuous fiber-reinforced 3D printed structures. Composite Structures, 2016. 153: p. 866-875.

77. Ming, Y., et al., Fabrication of continuous glass fiber-reinforced dual-cure epoxy composites via UV-assisted fused deposition modeling. Composites Communications, 2020. 21: p. 100401.

78. Ming, Y., et al., Investigation on process parameters of $3 D$ printed continuous carbon fiber-reinforced thermosetting epoxy composites. Additive Manufacturing, 2020. 33: p. 101184.

79. Mohammadizadeh, M., et al., Creep behavior analysis of additively manufactured fiberreinforced components. The International Journal of Advanced Manufacturing Technology, 2018. 99(5): p. 1225-1234.

80. Mohammadizadeh, M., et al., 3D printed fiber reinforced polymer composites-Structural analysis. Composites Part B: Engineering, 2019. 175: p. 107112.

81. Mori, K.-i., T. Maeno, and Y. Nakagawa, Dieless forming of carbon fibre reinforced plastic parts using 3D printer. Procedia engineering, 2014. 81: p. 1595-1600.

82. Mosleh, N., A.M. Rezadoust, and S. Dariushi, Determining process-window for manufacturing of continuous carbon fiber-reinforced composite Using 3D-printing. Materials and Manufacturing Processes, 2021. 36(4): p. 409-418. 
83. Nabipour, M. and B. Akhoundi, An experimental study of FDM parameters effects on tensile strength, density, and production time of ABS/Cu composites. Journal of Elastomers \& Plastics, 2020: p. 0095244320916838.

84. Naranjo-Lozada, J., et al., Tensile properties and failure behavior of chopped and continuous carbon fiber composites produced by additive manufacturing. Additive Manufacturing, 2019. 26: p. 227-241.

85. O'Connor, H.J. and D.P. Dowling, Low-pressure additive manufacturing of continuous fiber-reinforced polymer composites. Polymer Composites, 2019. 40(11): p. 4329-4339.

86. Oztan, C., et al., Microstructure and mechanical properties of three dimensional-printed continuous fiber composites. Journal of Composite Materials, 2019. 53(2): p. 271-280.

87. Peng, Y., et al., Tailorable rigidity and energy-absorption capability of $3 D$ printed continuous carbon fiber reinforced polyamide composites. Composites Science and Technology, 2020. 199: p. 108337.

88. Prajapati, A.R., H.K. Dave, and H.K. Raval, An Experimental Study on Mechanical, Thermal and Flame-Retardant Properties of 3D-Printed Glass-Fiber-Reinforced Polymer Composites. Journal of Materials Engineering and Performance, 2021: p. 1-12.

89. Prajapati, A.R., H.K. Dave, and H.K. Raval, Effect of fiber volume fraction on the impact strength of fiber reinforced polymer composites made by FDM process. Materials Today: Proceedings, 2021.

90. Prajapati, A.R., H.K. Dave, and H.K. Raval, Effect of fiber reinforcement on the open hole tensile strength of 3D printed composites. Materials Today: Proceedings, 2021.

91. Pyl, L., K.-A. Kalteremidou, and D. Van Hemelrijck, Exploration of specimen geometry and tab configuration for tensile testing exploiting the potential of $3 D$ printing freeform shape 
continuous carbon fibre-reinforced nylon matrix composites. Polymer Testing, 2018. 71: p. 318-328.

92. Qiao, J., Y. Li, and L. Li, Ultrasound-assisted 3D printing of continuous fiber-reinforced thermoplastic (FRTP) composites. Additive Manufacturing, 2019. 30: p. 100926.

93. Quan, C., et al., 3D printed continuous fiber reinforced composite auxetic honeycomb structures. Composites Part B: Engineering, 2020. 187: p. 107858.

94. Sanei, S.H., A. Arndt, and R. Doles, Open hole tensile testing of 3D printed continuous carbon fiber reinforced composites. Journal of Composite Materials, 2020. 54(20): p. 26872695.

95. Sanei, S.H.R., et al. Mechanical Properties of 3D Printed Fiber Reinforced Thermoplastic. in ASME 2019 International Mechanical Engineering Congress and Exposition. American Society of Mechanical Engineers Digital Collection.

96. N. Sarvestani, A., et al. 3D printed composites with continuous carbon fiber reinforcements. in ASME International Mechanical Engineering Congress and Exposition. 2017. American Society of Mechanical Engineers.

97. Shi, K., et al., 3D printing Kevlar fiber layer distributions and fiber orientations into nylon composites to achieve designable mechanical strength. Additive Manufacturing, 2021. 39: p. 101882.

98. Shiratori, H., et al., Compressive strength degradation of the curved sections of 3D-printed continuous carbon fiber composite. Composites Part A: Applied Science and Manufacturing, 2021. 142: p. 106244. 
99. Sugiyama, K., et al., 3D printing of optimized composites with variable fiber volume fraction and stiffness using continuous fiber. Composites Science and Technology, 2020. 186: p. 107905.

100. Sugiyama, K., et al., 3D printing of composite sandwich structures using continuous carbon fiber and fiber tension. Composites Part A: Applied Science and Manufacturing, 2018. 113: p. 114-121.

101. Tian, X., et al., Recycling and remanufacturing of $3 D$ printed continuous carbon fiber reinforced PLA composites. Journal of cleaner production, 2017. 142: p. 1609-1618.

102. Tian, X., et al., Interface and performance of $3 D$ printed continuous carbon fiber reinforced PLA composites. Composites Part A: Applied Science and Manufacturing, 2016. 88: p. 198-205.

103. Todoroki, A., et al., Tensile property evaluations of 3D printed continuous carbon fiber reinforced thermoplastic composites. Advanced Composite Materials, 2020. 29(2): p. 147-162.

104. Touchard, F., et al., Interfacial adhesion quality in $3 D$ printed continuous CF/PA6 composites at filament/matrix and interlaminar scales. Composites Part B: Engineering, 2021: p. 108891.

105. Van Der Klift, F., et al., 3D printing of continuous carbon fibre reinforced thermo-plastic (CFRTP) tensile test specimens. Open Journal of Composite Materials, 2016. 6(01): p. 18.

106. Vaneker, T., Material extrusion of continuous fiber reinforced plastics using commingled yarn. Procedia CIRP, 2017. 66: p. 317-322.

107. Wang, K., et al., Simultaneous reinforcement of both rigidity and energy absorption of polyamide-based composites with hybrid continuous fibers by $3 D$ printing. Composite Structures, 2021. 267: p. 113854. 
108. Wang, X., et al., 3D Printing of Continuous Fiber Reinforced Low Melting Point Alloy Matrix Composites: Mechanical Properties and Microstructures. Materials, 2020. 13(16): p. 3463.

109. Wang, Y., et al., Process parameters and mechanical properties of continuous glass fiber reinforced composites-polylactic acid by fused deposition modeling. Journal of Reinforced Plastics and Composites, 2021: p. 0731684421998017.

110. Yang, C., et al., 3D printing for continuous fiber reinforced thermoplastic composites: mechanism and performance. Rapid Prototyping Journal, 2017.

111. Yao, X., et al., Evaluation of carbon fiber-embedded $3 D$ printed structures for strengthening and structural-health monitoring. Materials \& Design, 2017. 114: p. 424-432.

112. Yin, L., et al., Characterizations of continuous carbon fiber-reinforced composites for electromagnetic interference shielding fabricated by 3D printing. Applied Physics A, 2019. 125(4): p. 1-11.

113. Yu, T., et al., Tensile and flexural behaviors of additively manufactured continuous carbon fiber-reinforced polymer composites. Composite Structures, 2019. 225: p. 111147.

114. Zeng, C., et al., Bending performance and failure behavior of 3D printed continuous fiber reinforced composite corrugated sandwich structures with shape memory capability. Composite Structures, 2021. 262: p. 113626.

115. Zhang, J., et al., Performance of 3D-printed continuous-carbon-fiber-reinforced plastics with pressure. Materials, 2020. 13(2): p. 471.

116. Zhang, Y., et al., Prediction of deformation and failure behavior of continuous fiber reinforced composite fabricated by additive manufacturing. Composite Structures, 2021. 265: p. 113738. 
117. Chacón, J., et al., Additive manufacturing of PLA structures using fused deposition modelling: Effect of process parameters on mechanical properties and their optimal selection. Materials \& Design, 2017. 124: p. 143-157. 